\title{
A Weighted Biobjective Transformation Technique for Locating Multiple Optimal Solutions of Nonlinear Equation Systems
}

\author{
Wenyin Gong, Yong Wang, Member, IEEE, Zhihua Cai, and Shengxiang Yang, Senior Member, IEEE
}

\begin{abstract}
Due to the fact that a nonlinear equation system may contain multiple optimal solutions, solving nonlinear equation systems is one of the most important challenges in numerical computation. When applying evolutionary algorithms to solve nonlinear equation systems, two issues should be considered: i) how to transform a nonlinear equation system into a kind of optimization problem, and ii) how to develop an optimization algorithm to solve the transformed optimization problem. In this paper, we tackle the first issue by transforming a nonlinear equation system into a weighted biobjective optimization problem. By the above transformation, not only do all the optimal solutions of an original nonlinear equation system become the Pareto optimal solutions of the transformed biobjective optimization problem, but also their images are different points on a linear Pareto front in the objective space. In addition, we suggest an adaptive multiobjective differential evolution, the goal of which is to effectively locate the Pareto optimal solutions of the transformed biobjective optimization problem. Once these solutions are found, the optimal solutions of the original nonlinear equation system can also be obtained correspondingly. By combining the weighted biobjective transformation technique with the adaptive multiobjective differential evolution, we propose a generic framework for the simultaneous locating of multiple optimal solutions of nonlinear equation systems. Comprehensive experiments on 38 nonlinear equation systems with various features have demonstrated that our framework provides very competitive overall performance compared with several state-ofthe-art methods.
\end{abstract}

Index Terms-Nonlinear equation systems, transformation technique, evolutionary multiobjective optimization, differential evolution.

\section{INTRODUCTION}

Manuscript received September 28, 2016; revised December 9, 2016 accepted February 15, 2017. This work was supported in part by the National Natural Science Foundation of China under Grants 61673397, 61573324, and 61375066 , in part by the Innovation-driven Plan in Central South University (No. 2015CXS012 and No. 2015CX007), in part by the EU Horizon 2020 Marie Sklodowska-Curie Individual Fellowships (Project ID: 661327), in part by the Engineering and Physical Sciences Research Council of UK under Grant EP/K001310/1, and in part by the Hunan Provincial Natura Science Fund for Distinguished Young Scholars (Grant No. 2016JJ1018). (Corresponding author: Yong Wang.)

W. Gong and Z. Cai are with the School of Computer Science, China University of Geosciences, Wuhan 430074, China. (Email: wygong@cug.edu.cn; zhcai@cug.edu.cn)

Y. Wang is with the School of Information Science and Engineering, Central South University, Changsha 410083, China, and also with the Centre for Computational Intelligence (CCI), School of Computer Science and Informatics, De Montfort University, Leicester LE1 9BH, UK. (Email: ywang@ csu.edu.cn)

$\mathrm{S}$. Yang is with the Centre for Computational Intelligence (CCI), Schoo of Computer Science and Informatics, De Montfort University, Leicester LE1 9BH, UK. (Email: syang@dmu.ac.uk)
$\mathbf{A}$ nonlinear equation system (NES) can be formulated as follows:

$$
\left\{\begin{aligned}
e_{1}(\mathbf{x}) & =0 \\
\vdots & \\
e_{m}(\mathbf{x}) & =0
\end{aligned}\right.
$$

where $e_{i}(\mathbf{x})=0(i \in\{1, \ldots, m\})$ is the $i$ th equation, $m$ is the number of equations, $\mathbf{x}=\left(x_{1}, \ldots, x_{n}\right)^{T} \in \mathcal{S}$ is a decision vector containing $n$ decision variables, $\mathcal{S}=\prod_{j=1}^{n}\left[\underline{x}_{j}, \bar{x}_{j}\right]$ is the decision space, and $\underline{x}_{j}$ and $\bar{x}_{j}$ are the lower and upper bounds of $x_{j}$, respectively. In general, a NES contains at least one nonlinear equation. $\mathrm{x}^{*}$ is called an optimal solution of a NES if for every $i \in\{1, \ldots, m\}, e_{i}\left(\mathbf{x}^{*}\right)=0$.

It is common to face a considerable number of NESs in the fields of mathematics, science, and engineering [1]. A NES may frequently contain more than one optimal solution, especially when the number of decision variables is equal to or larger than that of the equations. In order to allow the decision maker to select the most preferred solution, the purpose of solving NESs is to simultaneously locate multiple optimal solutions in a single run, which is perhaps one of the most challenging tasks in numerical computation [2].

During the past two decades, solving NESs by evolutionary algorithms (EAs) has been gaining increasing attention from the evolutionary computation research community, mainly because of the fact that EAs work with a population of candidate solutions and have good potential to find multiple optimal solutions of a NES in a single run. In principle, solving NESs by EAs can be considered as a two-step procedure [3]. In the first step, it is necessary to design a transformation technique, with the aim of recasting a NES as a kind of optimization problem. In the next step, an optimization algorithm should be developed to solve the transformed optimization problem.

The current popular transformation techniques can be classified into three categories: i) single-objective optimizationbased transformation techniques [2], [4], [5], [6], [7], [8], [9], [10], [11]; ii) constrained optimization-based transformation techniques [12], [13]; and iii) multiobjective optimizationbased transformation techniques [3], [14], [15]. The first kind of transformation technique usually converts a NES into the following single-objective optimization problem:

$$
\min \sum_{i=1}^{m}\left|e_{i}(\mathbf{x})\right|
$$

or

$$
\min \sum_{i=1}^{m} e_{i}^{2}(\mathbf{x})
$$


In addition, a constrained optimization problem is always constructed in the second kind of transformation technique:

$$
\left\{\begin{array}{cc}
\min & \sum_{i=1}^{m}\left|e_{i}(\mathbf{x})\right| \\
\text { subject to } & e_{i}(\mathbf{x}) \geq 0, i=1, \ldots, m
\end{array}\right.
$$

These two kinds of transformation techniques have a common feature: only one objective function is involved. Due to this feature, EAs may merely focus on one of the optimal solutions in one run when solving Equations (2)-(4). In order to locate multiple optimal solutions simultaneously, some extra diversity preservation strategies should be incorporated into EAs, such as the repulsion strategy [16], [17], [18]. However, such extra diversity preservation strategies would inevitably introduce some complicated operators and/or problem-dependent parameters, which have a side effect on the generalization. More importantly, if a NES contains infinitely many optimal solutions, EAs are not capable of maintaining a set of representative optimal solutions under the single-objective framework.

In contrast, multiobjective optimization-based transformation techniques redefine a NES as a multiobjective optimization problem. Note that multiobjective optimization problems also include a set of optimal solutions known as the Pareto optimal solutions. Moreover, the task of solving multiobjective optimization problems is to find multiple Pareto optimal solutions in a single run. Obviously, the above similarities imply that multiobjectivization is quite promising for NESs. Along this line, several methods have been proposed. In [14], each equation represents an objective function, and thus, a NES is converted into an $m$-objective optimization problem. In [15], there are $(n+1)$ objective functions in the transformed optimization problem. It is evident that with regard to these two methods, the number of objective functions is relevant to the number of equations or decision variables, respectively. As a consequence, they may suffer from the curse of dimensionality (i.e., many-objective) with the drastic increase of $m$ and $n$. Unlike [14] and [15], Song et al. [3] presented a method named MONES, which transforms a NES into a biobjective optimization problem. In MONES, the first decision variable (i.e., $x_{1}$ ) is exploited to guarantee the conflict between the two objective functions. However, if several optimal solutions of a NES have the same value in the first decision variable, it is very likely that some of them will be lost during the evolution.

In this paper, we propose a weighted biobjective transformation technique (called WeB) for NESs based on our previous work (i.e., MONES) in [3]. WeB shares the same biobjective structure with MONES. Different from MONES, in $\mathrm{WeB}$ all the decision variables, rather than just the first decision variable, are linearly weighted to construct the two objective functions. By doing this, WeB is able to remedy the loss of some optimal solutions with the same value in the first decision variable. Indeed, WeB is a generalization of MONES Systematic experiments on 38 NESs with a wide range of features have demonstrated that this simple modification to MONES can produce significantly better results. WeB has the following advantages over other transformation techniques:

- Compared with other multiobjective optimization-based transformation techniques, WeB has the potential to map the optimal solutions of a NES into different points on the linear Pareto front in the objective space under the biobjective structure because of the random weights.

- Compared with both single-objective optimization-based and constrained optimization-based transformation techniques, WeB has the capability to provide a set of representative optimal solutions for the situation where a NES contains infinitely many optimal solutions.

Additionally, we suggest an adaptive multiobjective differential evolution (AMODE) to solve the transformed biobjective optimization problem effectively. Furthermore, by combining WeB with AMODE, we propose a generic framework for simultaneously locating multiple optimal solutions of NESs. It is empirically shown that the performance of our framework is highly competitive with a lot of well-established methods for NESs.

The rest of this paper is organized as follows. Section II introduces the related work. Section III presents WeB. Section IV describes AMODE. Meanwhile, Section IV gives the details of our generic framework for solving NESs. Section V provides the experimental results. Section VI discusses some issues in our framework. Section VII concludes this paper and points out some future research directions.

\section{THE RELATED WORK}

Utilizing multiobjective optimization-based transformation techniques to deal with NESs is a novel idea that has arisen in recent years and is the main focus of this paper. As pointed out previously, in this kind of transformation technique, a NES is transformed into a multiobjective optimization problem. After this transformation, it is generally expected that the objective functions of the transformed optimization problem are in conflict with each other. This property plays an important role, as it can ensure that the optimal solutions of a NES are the Pareto optimal solutions of the transformed optimization problem. Otherwise, it is very difficult to identify the relationship between the original NES and the transformed optimization problem.

Next, we will briefly introduce five representative approaches. Among them, the last two approaches are originally designed for handling multimodal optimization problems [19]. Multimodal optimization problems have the same formulation as single-objective optimization problems. Note, however, that they involve multiple optimal solutions. One may be interested in why the last two approaches can be used for NESs. It is because if a NES is transformed into Equation (2) or Equation (3), this NES is also a single-objective optimization problem with multiple optimal solutions, which means that a NES with single-objective structure is essentially equivalent to a multimodal optimization problem. Thus, the multiobjective optimization-based transformation techniques, which convert a multimodal optimization problem into a multiobjective optimization problem, can be readily extended for coping with NESs.

\section{A. $C A$}

In 2008, Grosan and Abranham [14] proposed a new approach for NESs, called CA. By treating each equation as an 


$$
\left\{\begin{array}{c}
\min f_{1}(\mathbf{x})=\frac{x_{1}}{n}+\frac{x_{2}}{n-1}+\cdots+\frac{x_{n-1}}{2}+\frac{x_{n}}{1}+C \times R(\mathbf{x}) \times \ln (n+2) \\
\min f_{2}(\mathbf{x})=\frac{x_{1}}{n}+\frac{x_{2}}{n-1}+\cdots+\frac{x_{n-1}}{2}+\left(1-x_{n}\right)+C \times R(\mathbf{x}) \times \ln (n+1) \\
\min f_{3}(\mathbf{x})=\frac{x_{1}}{n}+\frac{x_{2}}{n-1}+\cdots+\frac{x_{n-2}}{3}+\left(1-x_{n-1}\right)+C \times R(\mathbf{x}) \times \ln (n) \\
\vdots \\
\min f_{n}(\mathbf{x})=\frac{x_{1}}{n}+\left(1-x_{2}\right)+C \times R(\mathbf{x}) \times \ln (3) \\
\min f_{n+1}(\mathbf{x})=\left(1-x_{1}\right)+C \times R(\mathbf{x}) \times \ln (2)
\end{array}\right.
$$

objective function, CA converts a NES into the following $m$ objective optimization problem:

$$
\left\{\begin{aligned}
\min f_{1}(\mathbf{x}) & =\left|e_{1}(\mathbf{x})\right| \\
\vdots & \\
\min f_{m}(\mathbf{x}) & =\left|e_{m}(\mathbf{x})\right|
\end{aligned}\right.
$$

CA provides a simple and straightforward transformation from a NES to a multiobjective optimization problem. However, the main drawback of CA is that its performance will significantly degrade as the number of equations increases [20].

\section{B. MONES}

In 2015, Song et al. [3] proposed MONES, which is a biobjective formulation for NESs. MONES consists of two parts: the location function and the system function. The former can be expressed as:

$$
\begin{cases}\min & \alpha_{1}(\mathbf{x})=x_{1} \\ \min & \alpha_{2}(\mathbf{x})=1-x_{1}\end{cases}
$$

where $x_{1}$ is the first decision variable of a NES. It is easily deduced that the Pareto front of Equation (6) is a line segment defined by $y=1-x$ in the objective space. In addition, the latter can be formulated as:

$$
\begin{cases}\min & \beta_{1}(\mathbf{x})=\sum_{i=1}^{m}\left|e_{i}(\mathbf{x})\right| \\ \min & \beta_{2}(\mathbf{x})=m \times \max _{i=1, \ldots, m}\left(\left|e_{1}(\mathbf{x})\right|, \ldots,\left|e_{m}(\mathbf{x})\right|\right)\end{cases}
$$

The transformed biobjective optimization problem can be obtained by combining these two parts:

$$
\begin{cases}\min & f_{1}(\mathbf{x})=\alpha_{1}(\mathbf{x})+\beta_{1}(\mathbf{x}) \\ \min & f_{2}(\mathbf{x})=\alpha_{2}(\mathbf{x})+\beta_{2}(\mathbf{x})\end{cases}
$$

It is interesting to note that for any optimal solution $\mathrm{x}^{*}$ of a NES, $\beta_{1}\left(\mathbf{x}^{*}\right)=\beta_{2}\left(\mathbf{x}^{*}\right)=0$, which means that under this condition Equation (8) degenerates to Equation (6). As a result, all the optimal solutions of a NES are the Pareto optimal solutions of Equation (8), and their images in the objective space are located on the line segment defined by $y=1-x$. In multiobjective optimization, linear Pareto front is the simplest type and, consequently, it enables the current multiobjective EAs to find the Pareto optimal solutions more easily compared with other types of Pareto front, such as nonlinear Pareto front [3]. However, since only the first decision variable is chosen to construct the location function, if several optimal solutions have the same value in the first decision variable, MONES might lose some of them.

\section{The Qin et al's Method}

Inspired by MONES [3], Qin et al. [15] presented a $(n+1)$ objective transformation technique in 2015, where $n$ is the number of decision variables. This transformation technique is also composed of two parts: the location function and the system function, which is shown in Equation (9). In Equation (9), $R(\mathbf{x})$ is the system function which is the mean of the absolute values of all equations, and $C$ is a parameter to control the shape of the Pareto front, which increases from 0 to infinity during the evolution.

In [15], all the objective functions in the location function conflict with each other, and thus, the optimal solutions of a NES are the Pareto optimal solutions of Equation (9). Moreover, the location function is able to provide a one-toone mapping from the Pareto optimal set to the Pareto front, thereby overcoming the drawback of MONES to a certain degree. However, similar to CA, this transformation technique will also suffer from the curse of dimensionality with the increase of $n$.

\section{D. $M O M M O P$}

In 2015, Wang et al. [21] developed a method named MOMMOP to deal with multimodal optimization problems, by generalizing the idea of MONES. Recognizing the shortcoming of MONES, MOMMOP makes use of each decision variable to construct a biobjective optimization problem like Equation (8) and, therefore, $n$ biobjective optimization problems appear. Because of the similarity between NESs and multimodal optimization problems, we revise MOMMOP to solve NESs as follows:

$$
\left\{\begin{aligned}
\mathrm{BOP}_{1} \begin{cases}\min & x_{1}+\beta_{1}(\mathbf{x}) \\
\min & 1-x_{1}+\beta_{2}(\mathbf{x})\end{cases} \\
\mathrm{BOP}_{n} \begin{cases}\min & x_{n}+\beta_{1}(\mathbf{x}) \\
\min & 1-x_{n}+\beta_{2}(\mathbf{x})\end{cases}
\end{aligned}\right.
$$

In MOMMOP, when comparing two individuals (denoted as $\mathbf{x}_{u}$ and $\mathbf{x}_{v}$ ), we say $\mathbf{x}_{u}$ is better than $\mathbf{x}_{v}$ if $\mathbf{x}_{u}$ Pareto dominates $\mathbf{x}_{v}$ on all the $n$ biobjective optimization problems in Equation (10):

$$
\left(\mathbf{x}_{u} \prec \mathbf{x}_{v} \text { on } \mathrm{BOP}_{1}\right) \wedge \cdots \wedge\left(\mathbf{x}_{u} \prec \mathbf{x}_{v} \text { on } \mathrm{BOP}_{n}\right)
$$

Compared with MONES, MOMMOP achieves the performance improvement at the expense of higher computational time complexity. Additionally, it is hard to analyze the property of the Pareto front theoretically since there exist $n$ twodimensional objective spaces in MOMMOP. 


\section{E. $M O B i D E$}

In 2013, Basak et al. [22] designed a biobjective differential evolution for multimodal optimization problems, abbreviated as MOBiDE. In MOBiDE, the first objective function aims to select the individuals of higher quality, and the purpose of the second objective function is to maintain the diversity to prevent the population from converging toward a single optimal solution. These two objective functions can be easily borrowed to solve NESs. For example, each individual $\mathbf{x}_{i}$ $(i \in\{1, \ldots, N P\})$ in the population is associated with the following two objective functions for NESs, where $N P$ is the population size:

$$
\begin{cases}\min & f_{1}\left(\mathbf{x}_{i}\right)=\sum_{k=1}^{m}\left|e_{k}\left(\mathbf{x}_{i}\right)\right| \\ \min & f_{2}\left(\mathbf{x}_{i}\right)=-\frac{\Omega_{i}}{N P}\end{cases}
$$

where $\Omega_{i}=\sum_{j=1}^{N P}\left\|\mathbf{x}_{i}-\mathbf{x}_{j}\right\|,\left\|\mathbf{x}_{i}-\mathbf{x}_{j}\right\|$ denotes the Euclidean distance between $\mathbf{x}_{i}$ and $\mathbf{x}_{j}$ in the decision space, and $f_{2}\left(\mathbf{x}_{i}\right)$ represents the average Euclidean distance from $\mathbf{x}_{i}$ to all other members in the population.

However, the limitation of MOBiDE is that the two objective functions are not totally in conflict with each other, which leads to an unclear relationship between the optimal solutions of a NES and the Pareto optimal solutions of Equation (12).

Remark 1: Apart from MOMMOP [21] and MOBiDE [22], many other biobjective transformation techniques for multimodal optimization problems have been proposed, see for example [23], [24], and [25]. We can take advantage of these transformation techniques to solve NESs. Note, however, that they have a similar disadvantage as MOBiDE. Consequently, their capabilities for finding multiple optimal solutions of a NES in a single run are limited. Due to the space limitation, we omit them in this paper.

\section{A Weighted Biobjective Transformation TECHNIQUE}

\section{A. Motivation}

Based on the above introduction, it is clear that multiobjective optimization-based transformation techniques, due to the similarity between NESs and multiobjective optimization problems, provide a natural advantage over other kinds of transformation techniques for NESs. As analyzed in Section II, in order to obtain competitive performance, the following four properties deserve much attention in this kind of transformation technique:

1) Biobjective structure, which has the least number of objective functions in multiobjective optimization problems, i.e., two.

2) Conflicting objective functions, which enable the optimal solutions of a NES to become the Pareto optimal solutions of the transformed multiobjective optimization problem.

3) Linear Pareto front, which is the simplest type of Pareto front for a multiobjective EA to approximate.

4) One-to-one mapping, which assures that all the optimal solutions of a NES are mapped into different points on the Pareto front in the objective space.
Interestingly, MONES possesses the first three properties. In this paper, we present a simple yet effective weighted biobjective transformation technique (WeB) that preserves the essential details of MONES while eliminating the effect of the fourth property.

\section{B. $W e B$}

In $\mathrm{WeB}$, the location function is defined as:

$$
\begin{cases}\min & \gamma_{1}(\mathbf{x})=\frac{\sum_{i=1}^{n} w_{i} \times x_{i}}{\sum_{i=1}^{n} w_{i}} \\ \min & \gamma_{2}(\mathbf{x})=1-\frac{\sum_{i=1}^{n} w_{i} \times x_{i}}{\sum_{i=1}^{n} w_{i}}\end{cases}
$$

where $\mathbf{w}=\left(w_{1}, \ldots, w_{n}\right)$ is the weight vector, and $w_{i}$ is the $i$ th weight randomly chosen from 0 and 1 . With respect to the location function, we can give the following comments:

- Equation (13) produces a weighted linear combination on all the decision variables.

- The two objective functions totally conflict with each other.

- The Pareto front is a line segment defined by $y=1-x$.

- If $w_{1} \neq 0$ and $\left(w_{2}, \ldots, w_{n}\right)=(0, \ldots, 0)$, the location function of $\mathrm{WeB}$ is equivalent to that of MONES, which means MONES is just a special case of WeB.

In addition, the system function is the same with Equation (2) and $\beta_{1}(\mathbf{x})$ in Equation (7) of MONES. It is because we would like to make the implementation and formulation as simple as possible.

The weighted biobjective optimization problem can be obtained by combining the location function with the system function:

$$
\begin{cases}\min & f_{1}(\mathbf{x})=\frac{\sum_{i=1}^{n} w_{i} \times x_{i}}{\sum_{i=1}^{n} w_{i}}+\sum_{i=1}^{m}\left|e_{i}(\mathbf{x})\right| \\ \min & f_{2}(\mathbf{x})=1-\frac{\sum_{i=1}^{n} w_{i} \times x_{i}}{\sum_{i=1}^{n} w_{i}}+\sum_{i=1}^{m}\left|e_{i}(\mathbf{x})\right|\end{cases}
$$

For an optimal solution $\mathrm{x}^{*}$ of a NES, Equation (14) will degenerate to Equation (13) since $\sum_{i=1}^{m}\left|e_{i}\left(\mathbf{x}^{*}\right)\right|=0$, which signifies that all the optimal solutions of a NES are the Pareto optimal solutions of $\mathrm{WeB}$ and that the Pareto front of $\mathrm{WeB}$ is linear.

From the previous description, one can conclude that the implementation of WeB is very simple and it does not introduce any problem-dependent parameters. Moreover, WeB keeps the main properties of MONES, i.e., biobjective structure, conflicting objective functions, and linear Pareto front.

The major difference between $\mathrm{WeB}$ and its predecessor MONES is that in $\mathrm{WeB}$, all the decision variables are utilized to design the location function in a linearly weighted fashion. As pointed out previously, MONES might fail to achieve a one-to-one mapping from the optimal set of a NES to the Pareto front, in the case of some optimal solutions having the same value in the first decision variable. That is, the optimal solutions with the same value in the first decision variable will be mapped into the same point on the Pareto front. However, regarding $\mathrm{WeB}$, the probability that the optimal solutions with the same values in certain decision variables or completely different values in all the decision variables are mapped into the same point on the Pareto front is very low because of the random weights. Therefore, $\mathrm{WeB}$ also has the fourth property mentioned in III-A. 


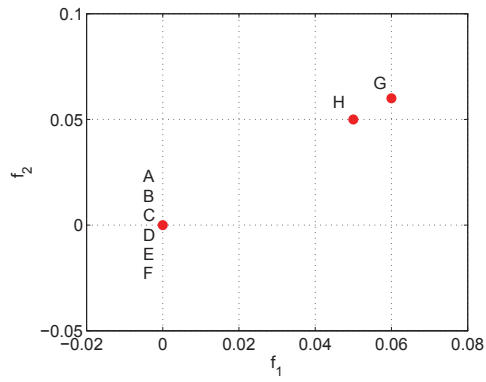

(a)

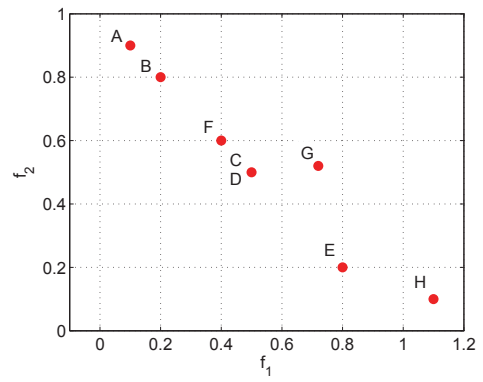

(b)

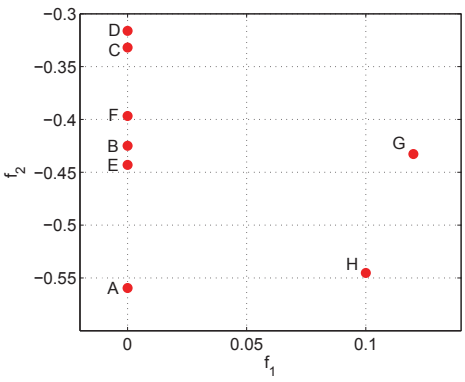

(c)

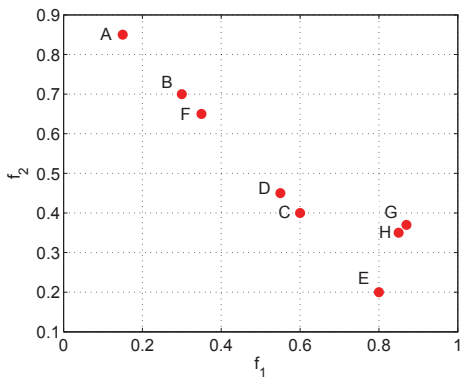

(d)

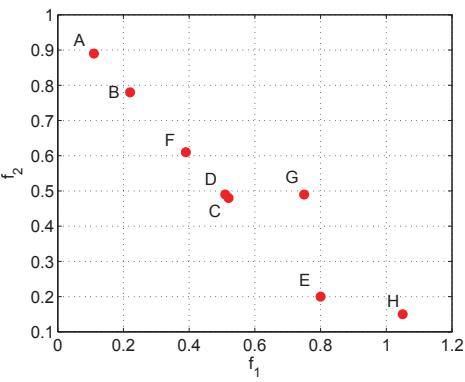

(e)

Fig. 1. The images of the eight individuals in the objective spaces defined by five different multiobjective optimization-based transformation techniques for the example in Table I. (a) CA [14]; (b) MONES [3]; (c) MOBiDE [22]; (d) WeB with w $=(0.5,0.5)$, and (e) WeB with w $=(0.9,0.1)$.

TABLE I

EIGHT INDIVIDUALS IN THE EXAMPLE

\begin{tabular}{|r|r|r|r|r|}
\hline \hline Solution & $x_{1}$ & $x_{2}$ & $e_{1}$ & $e_{2}$ \\
\hline $\mathrm{A}$ & 0.1 & 0.2 & 0 & 0 \\
\hline $\mathrm{B}$ & 0.2 & 0.4 & 0 & 0 \\
\hline $\mathrm{C}$ & 0.5 & 0.7 & 0 & 0 \\
\hline $\mathrm{D}$ & 0.5 & 0.6 & 0 & 0 \\
\hline $\mathrm{E}$ & 0.8 & 0.8 & 0 & 0 \\
\hline $\mathrm{F}$ & 0.4 & 0.3 & 0 & 0 \\
\hline $\mathrm{G}$ & 0.6 & 0.9 & 0.06 & 0.06 \\
\hline $\mathrm{H}$ & 1.0 & 0.5 & -0.05 & -0.05 \\
\hline \hline
\end{tabular}

\section{Analysis of the Principle}

An example with two decision variables is considered to illustrate the working principles of five different multiobjective optimization-based transformation techniques, i.e., CA [14], MONES [3], MOMMOP [21], MOBiDE [22], and WeB. As introduced in Section II, the Qin et al.'s method [15] is also a multiobjective optimization-based transformation technique. However, this method depends on a dynamic control parameter $C$, and it is not trivial to analyze its performance.

As shown in Table I, this example involves two equations $\left(e_{1}\right.$ and $\left.e_{2}\right)$. Suppose that there are eight individuals (denoted as $\mathrm{A}, \mathrm{B}, \mathrm{C}, \mathrm{D}, \mathrm{E}, \mathrm{F}, \mathrm{G}$, and $\mathrm{H}$ ) in the population. Among them, six individuals (i.e., A, B, C, D, E, and F) are the optimal solutions since all the values of the two equations are equal to zero, and the remaining two individuals (i.e., $\mathrm{G}$ and $\mathrm{H}$ ) are not the optimal solutions since the absolute values of the two equations are greater than zero. Fig. 1 depicts the images of these eight individuals in the objective spaces defined by CA, MONES, MOBiDE, and WeB. Note that there are $n$ biobjective optimization problems in MOMMOP, so we cannot provide a visualized result for it in the two-dimensional objective space.

Suppose also that the task is to select six individuals from the population for the next generation based on nondominated sorting [26]. Next, we are interested in what happens to these five multiobjective optimization-based transformation techniques.

- In CA, all the optimal solutions of a NES are mapped into the origin (i.e., $(0, \cdots, 0))$ in the objective space since the objective function values of all the optimal solutions are equal to zero based on Equation (5). As shown in Fig. 1(a), the images of the six optimal solutions lie in the origin. These six optimal solutions are the nondominated solutions in the population and will survive into the next generation.

- With respect to MONES, C and D are mapped into the same point in the objective space, and seven individuals (six optimal solutions and $\mathrm{H}$ ) are the nondominated solutions as shown in Fig. 1(b). Among these seven individuals, $\mathrm{C}$ and $\mathrm{D}$ have the smallest crowding distance [26]. Thus, A, B, C (or D), E, F, and $\mathrm{H}$ will be selected into the next generation. Clearly, one of the optimal solutions (i.e., C or D) is missed.

- For MOMMOP, after a careful analysis, all the individuals in the population are the nondominated solutions according to Equation (10). Subsequently, we compute their crowding distances in the decision space [21], and find that two of the optimal solutions (C and D) have the smallest crowding distance and will be removed during the selection.

- In terms of MOBiDE, all the optimal solutions are 
mapped into different points in the objective space and the population can be divided into six levels of nondominated set as shown in Fig. 1(c): $\{A\},\{E, H\},\{B, G\},\{F\}$, $\{C\}$, and $\{D\}$. As a result, $A, B, E, F, G$, and $H$ are the individuals with the most potential to be selected and two optimal solutions (C and D) are lost.

- To implement $\mathrm{WeB}$, all the weights in the weight vector $\mathbf{w}$ are randomly generated from 0 and 1 . In order to analyze the effect of the weight vector $\mathbf{w}$, we vary the weights with a step-size equal to 0.1 and obtain the following 11 weight vectors: $(0.0,1.0),(0.1,0.9),(0.2$, $0.8),(0.3,0.7),(0.4,0.6),(0.5,0.5),(0.6,0.4),(0.7$, $0.3),(0.8,0.2),(0.9,0.1)$, and $(1.0,0.0)$. According to our observation, all the optimal solutions correspond to different points in the objective space for all the weight vectors with the exception of $\mathbf{w}=(1.0,0.0)$. It is because WeB with $\mathbf{w}=(1.0,0.0)$ is roughly equivalent to MONES, thereby exhibiting the same drawback. In addition, for all the weight vectors except $\mathbf{w}=(1.0,0.0)$ and $\mathbf{w}=(0.9,0.1)$, the nondominated solutions are just the six optimal solutions, which will be chosen for the next generation. For example, the experimental results of WeB with $\mathbf{w}=(0.5,0.5)$ are given in Fig. 1(d). However, WeB with both $\mathbf{w}=(1.0,0.0)$ and $\mathbf{w}=(0.9,0.1)$ tend to lose one of the optimal solutions (i.e., $\mathrm{C}$ or $\mathrm{D}$ ). This can be attributed to the fact that in these two cases, the nondominated solutions consist of seven individuals (the six optimal solutions and $\mathrm{H}$ ), and one of $\mathrm{C}$ and $\mathrm{D}$ will be eliminated due to their having the smallest crowding distance. Fig. 1(e) shows the experimental results of WeB with $\mathbf{w}=(0.9,0.1)$.

We now summarize the above discussions from two aspects:

- MOBiDE can provide a one-to-one mapping from the set of the optimal solutions to the Pareto front. For 10 out of the 11 weight vectors, $\mathrm{WeB}$ is also able to achieve that. Unfortunately, it is a fact that CA will map all the optimal solutions into one point in the objective space and MONES will map the optimal solutions with the same value in the first decision variable into one point in the objective space.

- CA succeeds in selecting the six optimal solutions into the next generation ${ }^{1}$. For nine out of the 11 weight vectors, WeB can also do that. However, MONES, MOBiDE, and MOMMOP definitely lose some of the optimal solutions.

Overall, WeB seems to be the best choice: it offers a oneto-one mapping while maintaining the optimal solutions in a vast majority of cases. It is necessary to emphasize that in the practical implementation of $\mathrm{WeB}$, all the weights in the weight vector $\mathbf{w}$ are randomly generated. Thus, the probability that $\mathbf{w}=(1.0,0.0)$ is very low and the performance of WeB will be further enhanced.

\footnotetext{
${ }^{1}$ As mentioned in Section II-A, CA will suffer from the curse of dimensionality with the increase of the number of equations.
}

\section{An Adaptive Multiobjective Differential EVOLUTION}

As pointed out previously, when solving NESs by EAs, both the transformation technique and the optimization algorithm are vital. After transforming a NES into a biobjective optimization problem in Section III, the next issue is how to design an optimization algorithm to effectively solve the transformed biobjective optimization problem. To address this issue, we propose an adaptive multiobjective differential evolution, referred to as AMODE, which is an improved version of DEMO proposed in [27]. DEMO is mainly based on NSGA-II - a well-known multiobjective EA [26], whereas the search engine is replaced with differential evolution (DE) a very popular EA paradigm [28]. Due to its simple structure, ease of implementation, and better performance than NSGA$\mathrm{II}, \mathrm{DEMO}^{2}$ serves as the baseline optimization algorithm in AMODE. Moreover, two simple improvements are integrated within AMODE to make it more suitable for NESs as follows.

\section{A. Parameter Adaptation}

The performance of DE is significantly influenced by its parameter settings, such as the scaling factor $F$ and the crossover control parameter $C R$ [29], [30]. In this work, the parameter adaptation of AMODE originates from a very competitive DE variant, namely SHADE [31], in which a historical memory of successful parameter settings has been used to produce future parameter values.

In SHADE, each individual $\mathbf{x}_{i}(i \in\{1, \ldots, N P\})$ in the population has its own $F$ and $C R$, denoted as $F_{i}$ and $C R_{i}$. At each generation, the successful $F_{i}$ and $C R_{i}$ are stored into $\mathbf{S}_{\mathbf{F}}$ and $\mathbf{S}_{\mathbf{C R}}$, respectively. The main characteristic of SHADE is that it maintains a historical memory with $H$ entries for $\mathbf{M}_{\mathbf{F}}=$ $\left\{M_{F, 1}, \ldots, M_{F, H}\right\}$ and $\mathbf{M}_{\mathbf{C R}}=\left\{M_{C R, 1}, \ldots, M_{C R, H}\right\}$. The contents of $\mathbf{M}_{\mathbf{F}}$ and $\mathbf{M}_{\mathbf{C R}}$ are initialized to 0.5 and updated as follows:

$$
\begin{gathered}
M_{F, k}= \begin{cases}\operatorname{mean}_{W L}\left(\mathbf{S}_{\mathbf{F}}\right) & \text { if } \mathbf{S}_{\mathbf{F}} \neq \emptyset \\
M_{F, k} & \text { otherwise }\end{cases} \\
M_{C R, k}= \begin{cases}\operatorname{mean}_{W A}\left(\mathbf{S}_{\mathbf{C R}}\right) & \text { if } \mathbf{S}_{\mathbf{C R}} \neq \emptyset \\
M_{C R, k} & \text { otherwise }\end{cases}
\end{gathered}
$$

where $k \in\{1, \ldots, H\}$ determines the position to update, mean $_{W L}\left(\mathbf{S}_{\mathbf{F}}\right)$ is the weighted Lehmer mean of $\mathbf{S}_{\mathbf{F}}$, and mean $_{W A}\left(\mathbf{S}_{\mathbf{C R}}\right)$ is the weighted arithmetic mean of $\mathbf{S}_{\mathbf{C R}}{ }^{3}$. During the evolution, the value of $k$ increases generation by generation. If $k>H$, then it is reset to be 1 . In SHADE, the contents of $\mathbf{M}_{\mathbf{F}}$ and $\mathbf{M}_{\mathbf{C R}}$ are utilized to produce $F_{i}$ and $C R_{i}$ for the next generation.

${ }^{2}$ Note that three versions of DEMO are presented in [27], i.e., "DE$\mathrm{MO} /$ parent", "DEMO/closest/dec", and "DEMO/closest/obj". In this paper, "DEMO/closest/dec" is chosen because of its power to maintain high diversity of the population.

${ }^{3}$ Note that in Equation (15) the weighted Lehmer mean is used since it can generate larger scaling factors than the arithmetic mean [32]. In this way, the diversity of the population can be promoted. 


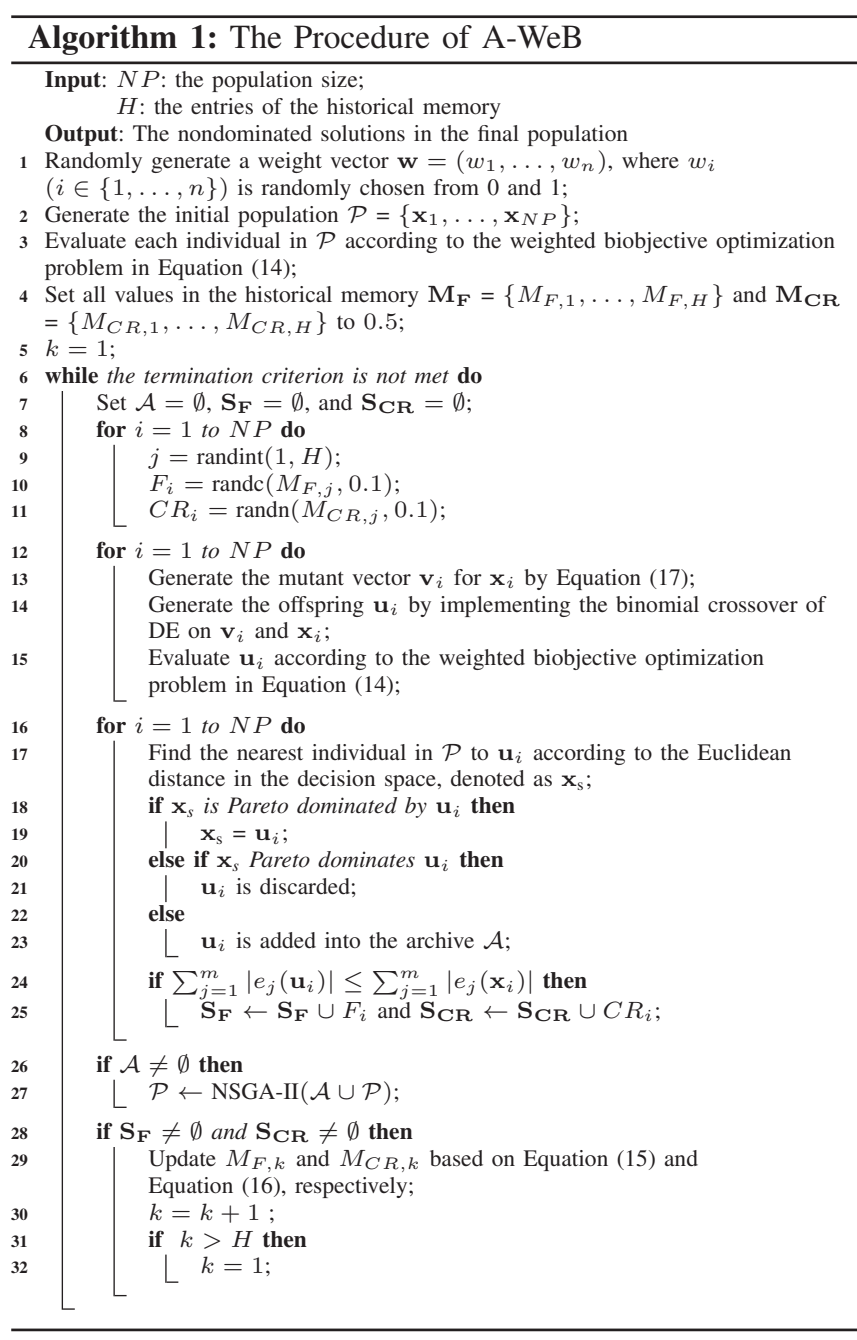

\section{B. Mutation Operator}

Several classical mutation operators are known in the DE research community. In this paper, we employ the mutation operator "DE/current/1" to create a mutant vector for each individual $\mathbf{x}_{i}(i \in\{1, \ldots, N P\})$ in the population:

$$
\mathbf{v}_{i}=\mathbf{x}_{i}+F_{i} \times\left(\mathbf{x}_{r_{1}}-\mathbf{x}_{r_{2}}\right)
$$

where $\mathbf{v}_{i}$ is the mutant vector, the indices $r_{1}$ and $r_{2}$ are two mutually distinct integers randomly selected from $\{1, N P\}$, and $F_{i}$ is a scaling factor between 0 and 1 .

"DE/current/1" is similar to a local search since the scaled difference of two individuals is directly added into the current individual $\mathbf{x}_{i}$. Thus, the search is carried out around the neighborhood of each individual, which facilitates the diversity of the population and provides an advantage to locate multiple optimal solutions. Note that "DE/current/1" has also attracted a lot of attention in multiobjective optimization [33], [34].

\section{Combining AMODE with WeB for Solving NESs}

By combining AMODE with $\mathrm{WeB}$, we propose a generic framework called A-WeB to simultaneously locate multiple optimal solutions of NESs, which is shown in Algorithm 1.
In each run of $\mathrm{A}-\mathrm{WeB}$, all the elements of the weight vector $\mathrm{w}$ are randomly generated and kept unchanged during the evolution, which means that each run has its own weight vector w. Additionally, all the individuals are evaluated according to the weighted biobjective optimization problem in Equation (14). During the evolution, firstly $F_{i}$ and $C R_{i}$ are produced for each individual $\mathbf{x}_{i}$ in the population $\mathcal{P}$ by lines $8-11$, where $\operatorname{randint}(1, H)$ is a randomly integer from 1 to $H, \operatorname{randc}(\cdot, \cdot)$ is a random number obeying a Cauchy distribution, and $\operatorname{randn}(\cdot, \cdot)$ is a random number obeying a Gaussian distribution ${ }^{4}$. Subsequently, the mutation operator "DE/current/1" and the binomial crossover of DE are used to yield an offspring $\mathbf{u}_{i}$ for each individual $\mathbf{x}_{i}$ in $\mathcal{P}$. Afterward, the nearest individual $\mathbf{x}_{\mathrm{S}}$ in $\mathcal{P}$ to $\mathbf{u}_{i}$ is identified and compared with $\mathbf{u}_{i}$ based on Pareto dominance. If $\sum_{j=1}^{m}\left|e_{j}\left(\mathbf{u}_{i}\right)\right| \leq \sum_{j=1}^{m}\left|e_{j}\left(\mathbf{x}_{i}\right)\right|$ which suggests that $F_{i}$ and $C R_{i}$ are the successful parameter settings, then they are stored into $\mathbf{S}_{\mathbf{F}}$ and $\mathbf{S}_{\mathbf{C R}}$, respectively. After the above update, NSGA-II [26] are utilized to choose $N P$ individuals from $\mathcal{P}$ and the archive $\mathcal{A}$. Finally, the contents of the historical memory $\mathbf{M}_{\mathbf{F}}$ and $\mathbf{M}_{\mathbf{C R}}$ are updated based on Equations (15) and (16), respectively. The above procedure is repeated until the termination criterion is met.

From the above explanations, it can be seen that the implementation of A-WeB is simple and its computational time complexity is the same with NSGA-II. In addition, it only contains two user-defined parameters, i.e., $N P$ and $H$.

\section{EMPIRICAL STUDIES}

Thirty-eight test instances with a broad range of characteristics, denoted as F01-F38, are used for our empirical studies. These 38 test instances are chosen from [3], [14], [35], [36], and [37], and can be divided into three classes:

- NESs with known optimal solutions (F01-F21), which include a number of optimal solutions.

- NESs with unknown optimal solutions (F22-F25), which include infinitely many optimal solutions.

- Ill-scaled NESs (F26-F38), in which the decision variables have different search ranges.

Table II summarizes the information of these 38 test instances and the details of them can be found in the supplemental file. For 15 test instances (i.e., F04, F07, F09, F12, F14, F15, F16, F18, F19, F20, F23, F24, F27, F30, and F38), some optimal solutions contain the same values in certain decision variables.

\section{A. Performance Metrics}

Based on [3] and [38], two performance metrics, i.e., peak ratio $(P R)$ and success rate $(S R)$, are used to assess the performance of a method for NESs with known optimal solutions

\footnotetext{
${ }^{4}$ In this paper, $F_{i}$ is generated obeying a Cauchy distribution and $C R_{i}$ is generated obeying a Gaussian distribution. The reasons are twofold. On one hand, recognizing the outstanding performance of SHADE [31], our parameter adaptation follows SHADE. On the other hand, the Cauchy distribution is more helpful to diversify $F_{i}$ than the Gaussian distribution [32]. Moreover, the Cauchy distribution coupled with the weighted Lehmer mean is more likely to produce larger values of $F_{i}$. Hence, under this condition the diversity of the population can be maintained, which is beneficial to find multiple optimal solutions of NESs simultaneously.
} 
TABLE II

Characteristics of 38 Test Instances, where $n$ is the Number of Decision Variables, $S$ is the Decision Space, $L I$ is the Number of Linear Equations, $N E$ is the Number of Nonlinear Equations, $N O S$ is the Number of Known Optimal Solutions of a NES, Max_FEs is the Maximal Number of Function Evaluations, and “ACtive Optimal Solutions" Indicates that Some Optimal Solutions Have the Same Values in Certain Decision Variables.

\begin{tabular}{|c|c|c|c|c|c|c|c|}
\hline Instance & $n$ & $\mathcal{S}$ & $L E$ & $N E$ & NOS & Max_FEs & Active Optimal Solutions \\
\hline F01 & 2 & {$[-1,1]^{n}$} & 1 & 1 & 2 & 50,000 & no \\
\hline F02 & 20 & {$[-1,1]^{n}$} & 0 & 2 & 2 & 50,000 & no \\
\hline F03 & 2 & {$[-1,1]^{n}$} & 1 & 1 & 11 & 50,000 & no \\
\hline F04 & 2 & {$[-1,1]^{n}$} & 0 & 2 & 15 & 50,000 & yes \\
\hline F05 & 2 & {$[-10,10]^{n}$} & 0 & 2 & 13 & 50,000 & no \\
\hline F06 & 10 & {$[-2,2]^{n}$} & 0 & 10 & 1 & 50,000 & no \\
\hline F07 & 2 & {$[-1,1]^{n}$} & 1 & 1 & 8 & 50,000 & yes \\
\hline F08 & 4 & {$[0,5]^{n}$} & 0 & 4 & 1 & 50,000 & no \\
\hline F09 & 2 & {$[0,1]^{n}$} & 0 & 2 & 7 & 50,000 & yes \\
\hline F10 & 5 & {$[-10,10]^{n}$} & 4 & 1 & 3 & 100,000 & no \\
\hline F11 & 6 & {$[-1,1]^{n}$} & 0 & 6 & 1 & 50,000 & no \\
\hline F12 & 2 & {$[-2,2]^{n}$} & 0 & 2 & 10 & 50,000 & yes \\
\hline F13 & 2 & {$[-5,5]^{n}$} & 0 & 2 & 9 & 50,000 & no \\
\hline F14 & 2 & {$[0,2 \pi]^{n}$} & 0 & 2 & 13 & 50,000 & yes \\
\hline F15 & 8 & {$[-1,1]^{n}$} & 0 & 8 & 16 & 100,000 & yes \\
\hline F16 & 2 & {$[-2,2]^{n}$} & 0 & 2 & 6 & 50,000 & yes \\
\hline F17 & 20 & {$[-2,2]^{n}$} & 19 & 1 & 2 & 200,000 & no \\
\hline F18 & 3 & {$[-1,1]^{n}$} & 0 & 3 & 7 & 50,000 & yes \\
\hline F19 & 2 & {$[-2,2]^{n}$} & 0 & 2 & 4 & 50,000 & yes \\
\hline F20 & 2 & {$[-2,2]^{n}$} & 0 & 2 & 6 & 50,000 & yes \\
\hline F21 & 3 & {$[0,1]^{n}$} & 0 & 3 & 8 & 100,000 & no \\
\hline F22 & 3 & {$[-1,1]^{n}$} & 1 & 1 & infinite & 50,000 & no \\
\hline F23 & 6 & {$[-1,1]^{n}$} & 0 & 6 & infinite & 50,000 & yes \\
\hline F24 & 20 & {$[-1,1]^{n}$} & 1 & 19 & infinite & 50,000 & yes \\
\hline F25 & 10 & {$[-10,10]^{n}$} & 4 & 6 & infinite & 50,000 & no \\
\hline F26 & 3 & {$[-5,5],[-1,3],[-5,5]$} & 0 & 3 & 2 & 50,000 & no \\
\hline F27 & 3 & {$[-0.6,6],[-0.6,0.6],[-5,5]$} & 0 & 3 & 12 & 50,000 & yes \\
\hline F28 & 2 & {$[0,1],[-10,0]$} & 0 & 2 & 2 & 50,000 & no \\
\hline F29 & 2 & {$[0,2.5],[-4,6]$} & 0 & 2 & 4 & 50,000 & no \\
\hline F30 & 2 & {$[-1,1],[-10,10]$} & 0 & 2 & 4 & 50,000 & yes \\
\hline F31 & 2 & {$[0.25,1],[1.5,2 \pi]$} & 0 & 2 & 2 & 50,000 & no \\
\hline F32 & 3 & {$[3,5],[2,4],[0.5,2]$} & 0 & 3 & 1 & 50,000 & no \\
\hline F33 & 2 & {$[-1,-0.1],[-2,2]$} & 0 & 2 & 2 & 50,000 & no \\
\hline F34 & 3 & {$[1,2.5],[0.2,2],[0.1,3]$} & 0 & 3 & 1 & 50,000 & no \\
\hline F35 & 2 & {$[-5,1.5],[0,5]$} & 0 & 2 & 3 & 50,000 & no \\
\hline F36 & 2 & {$[0,2],[10,30]$} & 0 & 2 & 2 & 50,000 & no \\
\hline F37 & 3 & {$[0,2],[-10,10],[-1,1]$} & 0 & 3 & 5 & 50,000 & no \\
\hline F38 & 2 & {$[-2,2],[0,1.1]$} & 0 & 2 & 4 & 50,000 & yes \\
\hline
\end{tabular}

(F01-F21) and ill-scaled NESs (F26-F38) in this paper. Prior to introducing these two performance metrics, we need to explain how to determine the number of the optimal solutions found in a run. Since the optimal solutions of these NESs are known a priori, firstly we choose one of the optimal solutions. If the minimum Euclidean distance between this optimal solution and all the solutions in the population obtained at the end of a run is less than a predefined accuracy level $\epsilon$, then we consider that an optimal solution is found. Afterward, the same process will be executed for the remaining optimal solutions one by one, and finally the number of the optimal solutions found can be calculated. If all the optimal solutions can be found in a run, then it is called a successful run. $P R$ denotes the average percentage of the optimal solutions found over all the runs and $S R$ denotes the percentage of the successful runs. In this paper, $\epsilon=0.01$ if $n \leq 5$, otherwise, $\epsilon=0.1$.

For NESs with unknown optimal solutions (F22-F25), these two performance metrics cannot be directly applied. Under this condition, the hypervolume metric [39] is used.

Note that for all the three performance metrics $(P R, S R$, and hypervolume), the larger the value, the better the performance of a method.

\section{B. Methods in Comparison and Experimental Setup}

We compare A-WeB with the following nine methods:

- A-MONES, A-MOMMOP, and A-MOBiDE: These three methods are obtained by combining AMODE with MONES [3], MOMMOP [21], and MOBiDE [22], respectively. MONES, MOMMOP, and MOBiDE have been introduced in Section II. Note that CA [14] is not chosen for comparison since its performance is outperformed by MONES as shown in [3]. In this paper, when implementing MONES, we randomly select a decision variable to construct the location function in each run.

- NCDE [40], NSDE [40], LIPS [41], and R3PSO [42]: These are four state-of-the-art niching methods designed for multimodal optimization problems. We pointed out in Section II that the methods for multimodal optimization problems can be easily generalized to handle NESs. When making use of NCDE, NSDE, LIPS, and R3PSO to solve NESs, a NES is transformed into the singleobjective optimization problem in Equation (2).

- Rep-SHADE and Rep-CLPSO: The repulsion strategy presented in [16] is combined with two powerful EAs, i.e., SHADE [31] and CLPSO [43], to solve NESs. The 
two resultant methods are referred to as Rep-SHADE and Rep-CLPSO. For Rep-SHADE and Rep-CLPSO, a NES is transformed into the following repulsion function once one optimal solution has been found:

$$
\min \sum_{i=1}^{m}\left|e_{i}(\mathbf{x})\right|+\beta \sum_{j=1}^{K} \mathrm{e}^{-\delta_{j}} \chi_{\rho}\left(\delta_{j}\right)
$$

where

$$
\begin{aligned}
\delta_{j} & =\left\|\mathbf{x}-\mathbf{x}_{j}^{*}\right\| \\
\chi_{\rho}\left(\delta_{j}\right) & = \begin{cases}1, & \text { if } \delta_{j} \leq \rho \\
0, & \text { otherwise }\end{cases}
\end{aligned}
$$

$K$ is the number of optimal solutions that have been found, $\mathbf{x}_{j}^{*}$ is the $j$ th optimal solution, $\delta_{j}$ is the Euclidean distance between $\mathbf{x}$ and $\mathbf{x}_{j}^{*}, \rho$ is a small constant to adjust the radius of repulsion areas, and $\beta$ is a large penalty constant. As suggested in [16], $\rho=0.01$ and $\beta=1000$. From Equation (18), we can see that the repulsion strategy creates the repulsion areas around the found optimal solutions and that an individual lies within one of the repulsion areas will be penalized. By doing this, the repulsion strategy has the potential to make the search algorithm find new optimal solutions.

It is evident that to achieve the simultaneous locating of multiple optimal solutions of a NES, among the aforementioned 10 methods, the first four methods (i.e., A-WeB, A-MONES, A-MOMMOP, and A-MOBiDE) integrate the multiobjective optimization-based transformation techniques with a multiobjective EA (AMODE), while the remaining six methods integrate the single-objective optimization-based transformation techniques with either the niching strategy or the repulsion strategy.

In our experiments, the parameter settings of A-WeB were: $N P=100$ and $H=N P$. For the other nine methods, $N P$ was also fixed to 100 and the other parameter settings were the same as in their original papers. Since AMOD$\mathrm{E}$ is also considered as the optimization algorithm in AMONES, A-MOMMOP, and A-MOBiDE, the parameter $H$ was fixed to $N P$ for them. The detailed parameter settings of the 10 compared methods were given in Table S-R-I of the supplemental file. Fifty independent runs were performed for each test instance with the maximal number of function evaluations (Max_FEs) as the termination criterion. Note that Max_FEs was set according to the difficulty of a NES as shown in Table II. To have a fair comparison, all the 10 methods started with the same initial population in each of 50 runs.

\section{Comparison on NESs with Known Optimal Solutions (F01- F21)}

The $P R$ and $S R$ values resulting from the 10 compared methods are summarized in Tables S-R-II and S-R-III of the supplemental file, respectively. Next, we will discuss the experimental results from the following four aspects:

- A-WeB performs the best in comparison with the other nine methods since it obtains both the highest average $P R$ value (0.8839) and the highest average $S R$ value
TABLE III

Results Obtained by the Multiple-Problem Wilcoxon Test For the 10 Compared Methods on F01-F21. $R^{+}$Means the Sum of RANKS That A-WeB PERForms Better than ITS COMPETITOR, AND $R^{-}$IS THE SUM OF RANKS FOR THE OpPosite.

\begin{tabular}{|r|c|c|c|c|c|c|}
\hline \hline \multirow{2}{*}{ A-WeB VS } & \multicolumn{3}{|c|}{$P R$} & \multicolumn{3}{c|}{$S R$} \\
\cline { 2 - 7 } & $R^{+}$ & \multicolumn{1}{|c|}{$R^{-}$} & $p$-value & $R^{+}$ & $R^{-}$ & $p$-value \\
\hline A-MONES & 220.0 & 11.0 & $\mathbf{5 . 2 5 E - 0 5}$ & 188.0 & 43.0 & $\mathbf{5 . 2 2 E - 0 3}$ \\
\hline A-MOMMOP & 169.5 & 61.5 & $\mathbf{4 . 4 1 E - 0 2}$ & 163.0 & 68.0 & $1.03 \mathrm{E}-01$ \\
\hline A-MOBiDE & 229.5 & 1.5 & $\mathbf{2 . 3 8 E - 0 6}$ & 219.0 & 12.0 & $\mathbf{4 . 7 7 E - 0 6}$ \\
\hline NCDE & 143.0 & 88.0 & $\geq 0.2$ & 138.0 & 93.0 & $\geq 0.2$ \\
\hline NSDE & 145.0 & 86.0 & $\geq 0.2$ & 143.0 & 88.0 & $\geq 0.2$ \\
\hline LIPS & 220.5 & 10.5 & $\mathbf{1 . 9 1 E - 0 6}$ & 229.5 & 1.5 & $\mathbf{2 . 3 8 E - 0 6}$ \\
\hline R3PSO & 231.0 & 0.0 & $\mathbf{9 . 5 4 E - 0 7}$ & 220.5 & 10.5 & $\mathbf{1 . 9 1 E - 0 6}$ \\
\hline Rep-SHADE & 141.0 & 90.0 & $\geq 0.2$ & 149.0 & 82.0 & $\geq 0.2$ \\
\hline Rep-CLPSO & 192.0 & 39.0 & $\mathbf{6 . 2 8 E - 0 3}$ & 172.0 & 59.0 & $\mathbf{3 . 4 5 E - 0 2}$ \\
\hline \hline
\end{tabular}

TABLE IV

RANKINGS OBtained By the Friedman Aligned TEST FOR THE 10 COMPAREd METHOdS ON F01-F21. THE LOWER THE RANKING, THE Better the Performance of a Method. The Best and the Second Best Results are Highlighted in Boldface and ItaliC, RESPECTIVELY.

\begin{tabular}{|r|c|c|}
\hline Method & Ranking $(P R)$ & Ranking $(S R)$ \\
\hline A-WeB & $\mathbf{5 0 . 6 6 6 7}$ & $\mathbf{5 6 . 0 0 0 0}$ \\
\hline A-MONES & 98.3810 & 94.6905 \\
\hline A-MOMMOP & 76.4762 & 81.1905 \\
\hline A-MOBiDE & 161.2381 & 157.0000 \\
\hline NCDE & 72.0238 & 80.2381 \\
\hline NSDE & 71.6905 & 78.4048 \\
\hline LIPS & 174.4524 & 165.8333 \\
\hline R3PSO & 189.8095 & 172.0000 \\
\hline Rep-SHADE & 70.4286 & 76.5000 \\
\hline Rep-CLPSO & 89.8333 & 93.1429 \\
\hline \hline
\end{tabular}

(0.64). In addition, A-WeB provides both the best $P R$ values and the best $S R$ values on 10 test instances (i.e., F01, F03, F05, F06, F08, F10, F11, F14, F18, and F20). A-WeB also achieves $100 \% P R$ and $100 \% S R$ on seven test instances (i.e., F01, F03, F05, F06, F11, F14, and F20), which means that it succeeds in locating all the optimal solutions over all 50 runs. Moreover, the $P R$ and $S R$ values derived from A-WeB are larger than zero for all the test instances except F15.

- A-MOMMOP, NCDE, NSDE, and Rep-SHADE show similar and competitive performance in terms of the average $P R$ and $S R$. They have the capability to successfully solve five, five, five, and seven NESs, respectively. Note that NCDE and Rep-SHADE fail to find any optimal solution on one (F10) and two (F02 and F08) test instances, respectively.

- Again, A-MONES and Rep-CLPSO present similar overall performance. However, they do not perform as well as the above five methods. They are able to locate all the optimal solutions of four and six test instances, respectively. Rep-CLPSO cannot find any optimal solution on three test instances (F02, F08, and F10).

- The performance of A-MOBiDE, LIPS, and R3PSO is found to decrease remarkably. A-MOBiDE and LIPS can achieve $100 \%$ successful runs on only two (F06 and F11) and one (F01) test instance, respectively. R3PSO cannot solve any test instance consistently in all runs and does not yield good performance in a vast majority of test instances. As mentioned in Section II, for A- 
TABLE V

The Average and Standard Deviation of the Hypervolume Values of the Seven Compared Methods on F22-F25.

\begin{tabular}{|r||l|l|l|l||c|}
\hline \hline & F22 & F23 & F24 & F25 & $+/=$ \\
\hline A-WeB & $0.296675 \pm 0.035995$ & $0.686763 \pm 0.007750$ & $0.039690 \pm 0.001414$ & $4.046544 \pm 0.359629$ & $/$ \\
\hline A-MONES & $0.288931 \pm 0.035115+$ & $0.606079 \pm 0.114344+$ & $0.027886 \pm 0.002659+$ & $3.140386 \pm 0.551425+$ & $4 / 0 / 0$ \\
\hline A-MOMMOP & $0.298077 \pm 0.036335-$ & $0.662113 \pm 0.008899+$ & $0.039333 \pm 0.000078=$ & $3.898406 \pm 0.244397+$ & $2 / 1 / 1$ \\
\hline NCDE & $0.291552 \pm 0.033778+$ & $0.678183 \pm 0.006296+$ & $0.031114 \pm 0.001102+$ & $2.611568 \pm 0.240187+$ & $4 / 0 / 0$ \\
\hline NSDE & $0.291554 \pm 0.033673+$ & $0.680569 \pm 0.006895+$ & $0.031185 \pm 0.001429+$ & $2.676923 \pm 0.219315+$ & $4 / 0 / 0$ \\
\hline Rep-SHADE & $0.296955 \pm 0.035146=$ & $0.683148 \pm 0.008029+$ & $0.034823 \pm 0.001041+$ & $2.531974 \pm 0.379190+$ & $3 / 1 / 0$ \\
\hline Rep-CLPSO & $0.300833 \pm 0.036073-$ & $0.677834 \pm 0.008074+$ & $0.034317 \pm 0.001290+$ & NA \pm NA + & $3 / 0 / 1$ \\
\hline \hline ref. point & $(1,1)$ & $(0.6,1.6)$ & $(0.1,1.2)$ & $(1.5,2.5)$ & $/$ \\
\hline
\end{tabular}

"+", "=", and "-" indicate that A-WeB is better than, similar to, and worse than its competitor according to the Wilcoxon signed-rank test at $\alpha=0.05$, respectively.

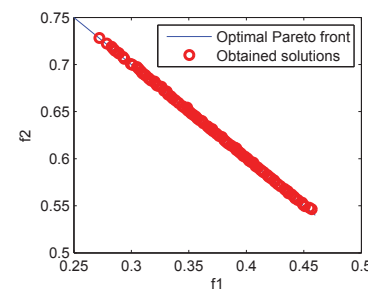

(a)

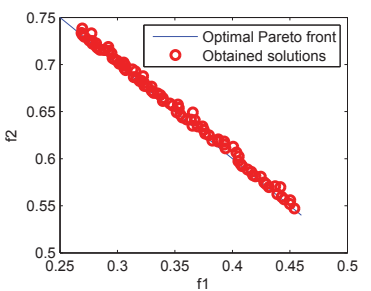

(b)

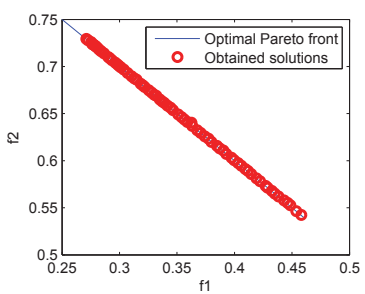

(c)

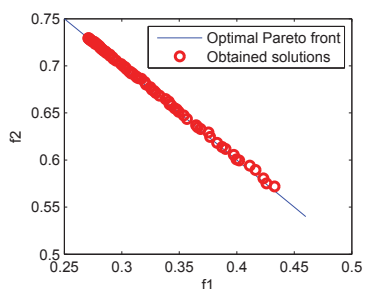

(d)

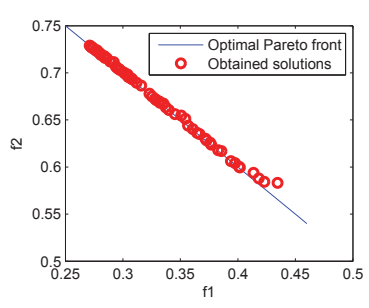

(e)

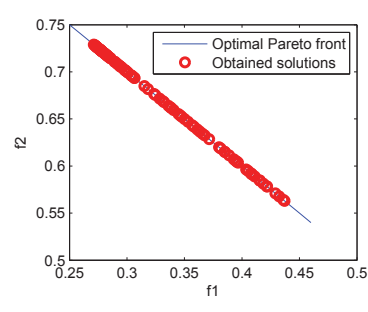

(f)

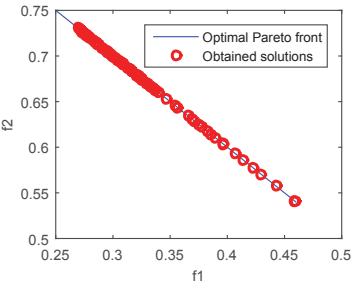

(g)

Fig. 2. The images of the obtained nondominated solutions of different methods in the objective space defined by Equation (14) for F22 in a representative run. The solid line is the theoretical Pareto front with $y=1-x$. (a) A-WeB; (b) A-MONES; (c) A-MOMMOP; (d) NCDE; (e) NSDE; (f) Rep-SHADE; and (g) Rep-CLPSO.

MOBiDE, the relationship between the optimal solutions of an original NES and the Pareto optimal solutions of the transformed biobjective optimization problem cannot be described explicitly. The poor performance of LIPS and R3PSO could be explained by the fact that they employ simple velocity update equation of PSO, and as a result, their search ability is limited.

We also tested the statistical differences of the 10 compared methods by making use of the multiple-problem Wilcoxon test and the Friedman Aligned test [44]. It is noteworthy that the statistical tests were implemented via the KEEL software [45]. Moreover, we chose the Bonferroni-Dunn method as the post-hoc test for the Friedman Aligned test. The statistical test results are given in Tables III and IV.

As shown in Table III, in terms of the multiple-problem Wilcoxon test, A-WeB provides higher $R^{+}$values than $R^{-}$ values in all the cases for both the $P R$ and $S R$ metrics. Especially, A-WeB significantly outperforms A-MONES, AMOBiDE, LIPS, R3PSO, and Rep-CLPSO in that all the $p$ values are less than 0.05 . In addition, it can be seen from Table IV that with respect to both the $P R$ and $S R$ metrics, $\mathrm{A}-\mathrm{WeB}$ has the best ranking, followed by Rep-SHADE.

\section{Comparison on NESs with Unknown Optimal Solutions (F22-F25)}

F22-F25 contain infinitely many optimal solutions. The $P R$ and $S R$ metrics are not suitable for evaluating the performance of a method on these four test instances. To complete the performance comparison, the hypervolume metric [39] was used. To make the comparison fair, the nondominated individuals in the final populations provided by different methods need to be mapped into the same objective space. In this paper, with the termination of each run, the nondominated individuals in the final populations provided by different methods are mapped into the two-dimensional objective space defined by Equation (14). Since the Pareto front in this objective space is a line segment defined by $y=1-x$, we need to measure how close the nondominated individuals converge toward the Pareto front and how uniformly the nondominated individuals distribute along the Pareto front, which are the two essential goals in multiobjective optimization. Fortunately, the hypervolume metric is effective in measuring both the convergence and uniformness.

Table $\mathrm{V}$ records the average and standard deviation of the hypervolume values derived from different methods on F22F25. In Table V, "NA" denotes that the experimental results 


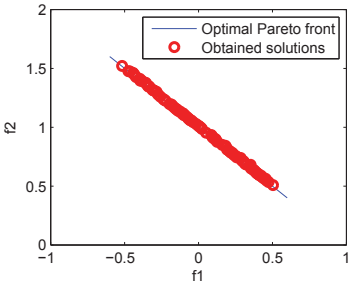

(a)

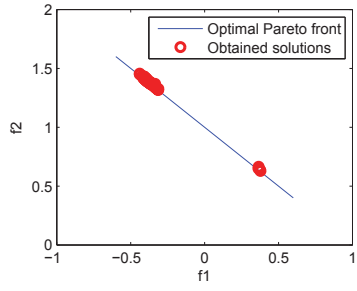

(b)

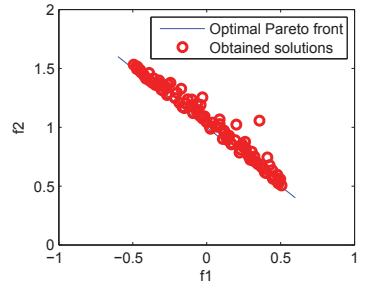

(c)

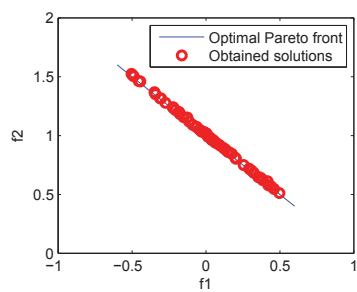

(d)

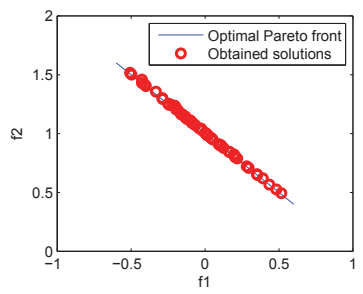

(e)

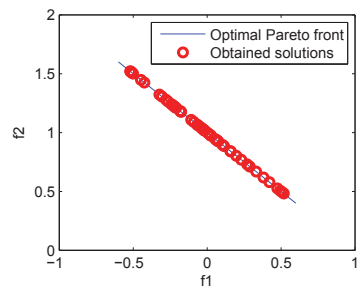

(f)

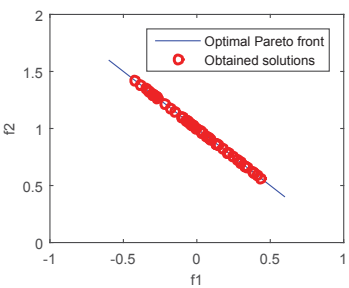

(g)

Fig. 3. The images of the obtained nondominated solutions of different methods in the objective space defined by Equation (14) for F23 in a representative run. The solid line is the theoretical Pareto front with $y=1-x$. (a) A-WeB; (b) A-MONES; (c) A-MOMMOP; (d) NCDE; (e) NSDE; (f) Rep-SHADE; and (g) Rep-CLPSO.

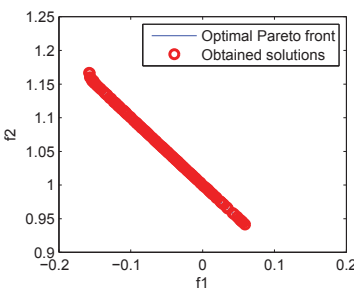

(a)

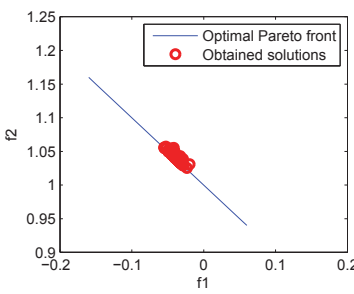

(b)

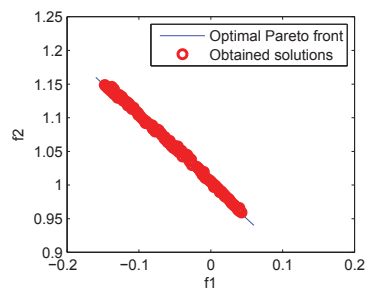

(c)

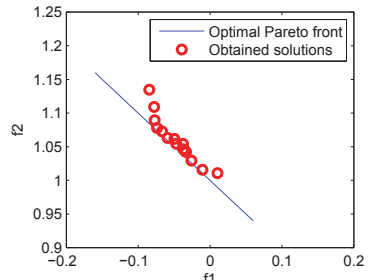

(d)

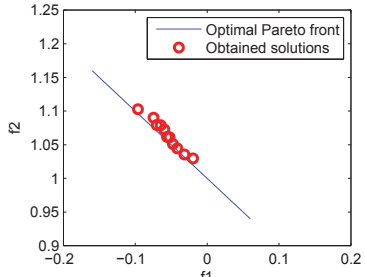

(e)

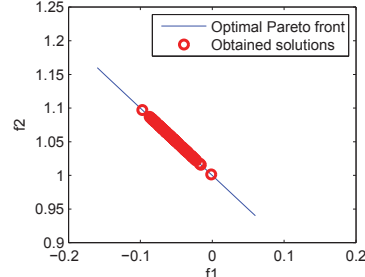

(f)

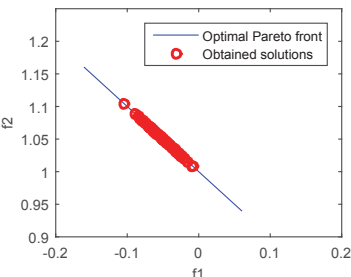

(g)

Fig. 4. The images of the obtained nondominated solutions of different methods in the objective space defined by Equation (14) for F24 in a representative run. The solid line is the theoretical Pareto front with $y=1-x$. (a) A-WeB; (b) A-MONES; (c) A-MOMMOP; (d) NCDE; (e) NSDE; (f) Rep-SHADE; and (g) Rep-CLPSO

of Rep-CLPSO are not available on F25 since Rep-CLPSO cannot find any optimal solution with the pre-specified accuracy level (i.e., 0.01) and the repulsion strategy is not triggered under this condition. Due to the fact that A-MOBiDE, LIPS, and R3PSO do not yield good performance in Section V-C, their experimental results are not included for convenience. To test the statistical significance between $\mathrm{A}-\mathrm{WeB}$ and each competitor, Wilcoxon signed-rank test at a 0.05 significance level was applied. As shown in Table V, A-WeB performs significantly better than A-MONES, A-MOMMOP, NCDE, NSDE, Rep-SHADE, and Rep-CLPSO on four, two, four, four, three, and three test instances, respectively. However, A-
MOMMOP and Rep-CLPSO have an edge over A-WeB on only one test instance (F22), and A-MONES, NCDE, NSDE, and Rep-SHADE cannot surpass A-WeB on any test instance. The above comparison confirms that, on the whole, A-WeB presents the best performance among the seven compared methods on four test instances with infinitely many optimal solutions.

Next, we further study the performance differences by observing the nondominated solutions in the final population. Figs. 2-5 provide the experimental results of the seven compared methods in a representative run. In this work, the run in which the hypervolume value of the nondominated solutions 


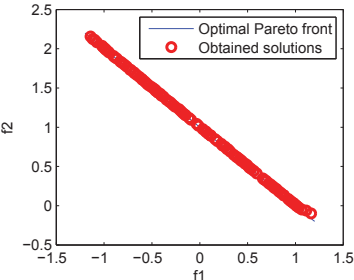

(a)

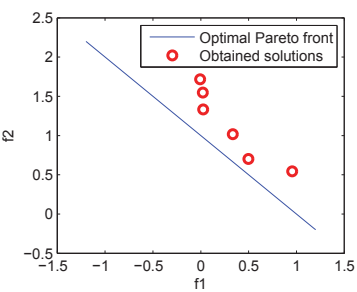

(d)

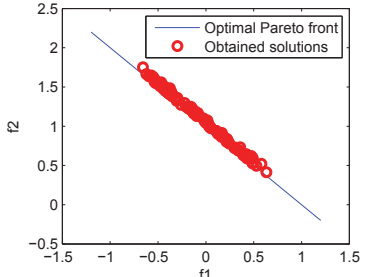

(b)

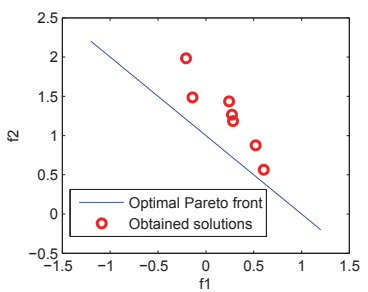

(e)

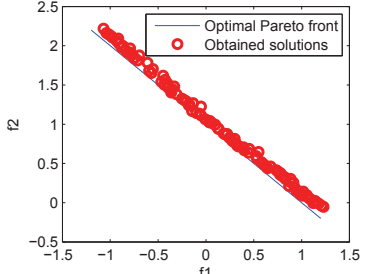

(c)

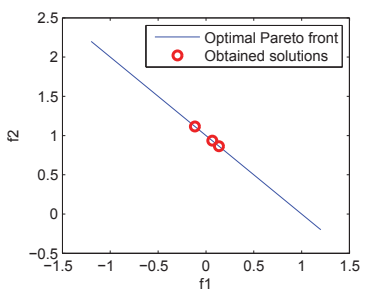

(f)

Fig. 5. The images of the obtained nondominated solutions of different methods in the objective space defined by Equation (14) for F25 in a representative run. The solid line is the theoretical Pareto front with $y=1-x$. (a) A-WeB; (b) A-MONES; (c) A-MOMMOP; (d) NCDE; (e) NSDE; and (f) Rep-SHADE.

in the final population ranks 26th (from worst to best) is termed as a representative run among 50 runs. It is easy to see that $\mathrm{A}-\mathrm{WeB}$ is able to consistently produce a set of representative nondominated solutions with good convergence and uniformness on F22-F25. Although the performance of AMOMMOP is better than that of A-Web on F22, A-MOMMOP is not as effective as A-WeB for approximating the Pareto front of F23 and F25. The reason could be that when comparing two individuals in MOMMOP, the Pareto dominance should be checked on all the $n$ biobjective optimization problems. If the Pareto dominance does not hold between them on any of the $n$ biobjective optimization problems, then they are considered to be nondominated. Thus, the population of MOMMOP might contain a lot of nondominated solutions, which leads to low selection pressure and slow convergence speed. As depicted in Figs. 3 and 4, A-MONES runs the risk of missing some parts of the Pareto front of F23 and F24, in which some optimal solutions have the same values in certain decision variables. The preformation degradation of A-MONES coincides with our analysis in Section II. Regarding the four single-objective optimization-based methods (NCDE, NSDE, Rep-SHADE, and Rep-CLPSO), they tend to yield decent performance when the number of decision variables is small (i.e., F22 and F23). But, as the number of decision variable increases (i.e., F24 and F25), they cannot obtain promising results in terms of the convergence and uniformness as shown in Figs. 4 and 5.

\section{E. Comparison on Ill-Scaled NESS (F26-F38)}

As for the previous test instances, the decision variables have the same search region. A question which arises naturally is how the performance of a method is influenced by the illscaled NESs, in which the search ranges of decision variables are different. To this end, we collect 13 ill-scaled NESs (F26F38) in Table II and the performance of different methods is compared on them. Like Section V-D, the experimental results
TABLE VI

Results Obtained By the Multiple-Problem Wilcoxon Test for THE SEVEn COMPAREd METHOdS ON F26-F38.

\begin{tabular}{|r|c|c|c|c|c|c|}
\hline \hline \multirow{2}{*}{ A-WeB VS } & \multicolumn{3}{|c|}{$P R$-value } & $R^{+}$ & $R^{-}$ & $p$-value \\
\cline { 2 - 7 } & $R^{+}$ & $R^{-}$ & $p$-vale- & 66.5 & 24.5 & $1.10 \mathrm{E}-01$ \\
\hline A-MONES & 73.0 & 18.0 & $5.74 \mathrm{E}-02$ & $\mathbf{3 . 4 2 E}-02$ \\
\hline A-MOMMOP & 65.0 & 26.0 & $1.91 \mathrm{E}-01$ & 72.5 & 18.5 & $\mathbf{3 . 7 4 E}-02$ \\
\hline NCDE & 66.0 & 25.0 & $1.68 \mathrm{E}-01$ & 71.0 & 20.0 & $\mathbf{4 . 7 4}$ \\
\hline NSDE & 48.5 & 42.5 & $\geq 0.2$ & 50.5 & 40.5 & $\geq 0.2$ \\
\hline Rep-SHADE & 41.0 & 50.0 & $\geq 0.2$ & 45.5 & 45.5 & $\geq 0.2$ \\
\hline Rep-CLPSO & 69.5 & 21.5 & $1.02 \mathrm{E}-01$ & 74.0 & 17.0 & $\mathbf{2 . 3 9 E - 0 2}$ \\
\hline \hline
\end{tabular}

TABLE VII

Rankings Obtained by the Friedman Aligned Test for the SEven Compared Methods on F26-F38. The Best and the Second Best Results aRe Highlighted in BoldFACE AND ItAlic, Respectively.

\begin{tabular}{|r|c|c|}
\hline \hline Method & Ranking $(P R)$ & Ranking $(S R)$ \\
\hline A-WeB & 39.5000 & $\mathbf{3 4 . 4 6 1 5}$ \\
\hline A-MONES & 46.5769 & 43.5385 \\
\hline A-MOMMOP & 53.6538 & 55.8846 \\
\hline NCDE & 52.2692 & 60.8077 \\
\hline NSDE & $\mathbf{3 5 . 9 2 3 1}$ & 36.1538 \\
\hline Rep-SHADE & 41.5000 & 36.8462 \\
\hline Rep-CLPSO & 52.5769 & 54.3077 \\
\hline
\end{tabular}

of A-MOBiDE, LIPS, and R3PSO are omitted.

Tables S-R-IV and S-R-V in the supplemental file summarize the $P R$ and $S R$ values provided by the seven compared methods, respectively. From Tables S-R-IV and S-R-V, it can be observed that A-WeB achieves the second best average $P R$ value, and the same best average $S R$ value with NSDE. Additionally, A-WeB provides the best results on nine out of 13 test instances for both the $P R$ and $S R$ metrics. It can successfully solve nine test instances over all 50 runs.

Tables VI and VII report the statistical test results based on the multiple-problem Wilcoxon test and the Friedman Aligned test, respectively. As shown in Table VI, A-WeB provides higher $R^{+}$values than $R^{-}$values when comparing with all the competitors except Rep-SHADE. Rep-SHADE provides 


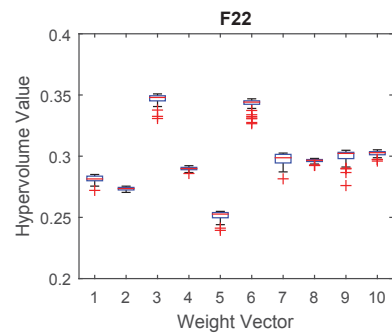

(a)

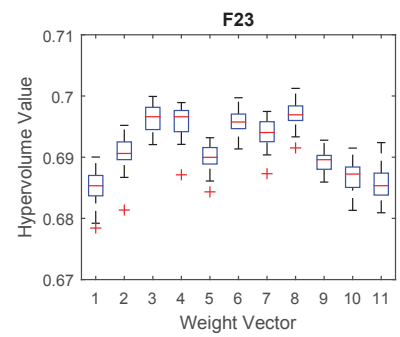

(b)

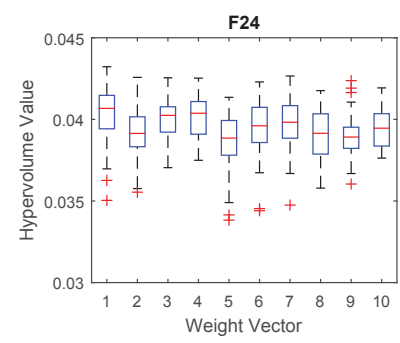

(c)

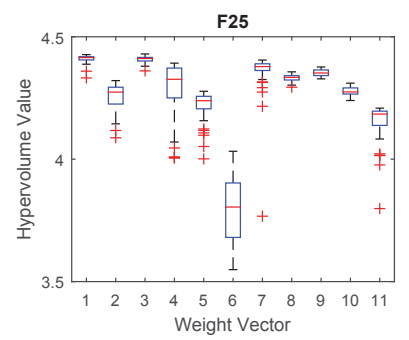

(d)

Fig. 6. The box plots of the hypervolume values derived from WeB with different weight vectors for F22-F25. (a) F22; (b) F23; (c) F24; and (d) F25.

higher $R^{-}$value than $R^{+}$value for $P R$, and the same $R^{+}$and $R^{-}$value for $S R$. As far as the multiple-problem Wilcoxon test at $\alpha=0.05$ is concerned, the significant differences can be observed in three cases (i.e., A-WeB versus A-MOMMOP, A-WeB versus NCDE, and A-WeB versus RepCLPSO) for $S R$, which suggests that under this condition A$\mathrm{WeB}$ is significantly better than A-MOMMOP, NCDE, and Rep-CLPSO. In addition, Table VII indicates that A-WeB ranks the second best and the best in terms of the $P R$ and $S R$ metrics, respectively. Therefore, on the whole, we can conclude that the performance of A-WeB is highly competitive on the 13 ill-scaled NESs.

Based on the discussions in the above three subsections, we can give the following remarks:

- WeB has the capability to alleviate the drawback of MONES when some optimal solutions have the same values in certain decision variables, which verifies the main motivation of this paper. We attribute the superiority of WeB to the fact that it uses the information of all the decision variables under the biobjective framework by random weights.

- The single-objective optimization-based methods with the niching or repulsion strategy (such as NCDE, NSDE, Rep-SHADE, and Rep-CLPSO) can find multiple optimal solutions of a NES in a single run. However, such strategies always introduce some user-defined parameters, which need to be set properly to solve different NESs More importantly, this kind of method has no specific diversity maintenance mechanism as in multiobjective EAs and, consequently, its performance is poor for the NESs with infinitely many optimal solutions, especially when the number of decision variables is high, which makes WeB more attractive.

- WeB is the best multiobjective optimization-based transformation technique compared with MONES, MOMMOP, and MOBiDE. A-WeB exhibits the best overall performance when solving different kinds of NESs.

\section{Discussions}

The aim of this section is to study the robustness of A$\mathrm{WeB}$, the scalability of $\mathrm{A}-\mathrm{WeB}$, the effectiveness of some mechanisms in $\mathrm{A}-\mathrm{WeB}$, and the influence of the parameter settings on the performance of A-WeB.

\section{A. On the Robustness of $A-W e B$}

In $\mathbf{A}-\mathrm{WeB}$, the weight vector $\mathbf{w}=\left(w_{1}, \ldots, w_{n}\right)$ in $\mathrm{E}$ quation (14) is randomly generated at the beginning of each run and is kept untouched during the evolution. Then, a straightforward question is how A-WeB performs with a fixed weight vector rather than a random weight vector over all 50 runs. Subsequently, we carried out experiments to answer this question. Specifically, we chose 11 weight vectors in which the first 10 weight vectors were randomly produced and the last weight vector contained equal elements for all the decision variables, i.e., $w_{1}=\cdots=w_{n}$. Fifty independent runs were implemented for A-WeB associated with each of the 11 weight vectors and test instances F22-F25 were used to produce the experimental results.

The box plots of the hypervolume values over 50 runs are shown in Fig. 6 for F22-F25. It is noteworthy that if $w_{1}=$ $\cdots=w_{n}$, then for any optimal solution $\mathbf{x}^{*}=\left(x_{1}^{*}, \ldots, x_{n}^{*}\right)$ of a NES, Equation (14) becomes:

$$
\begin{cases}\min & f_{1}(\mathbf{x})=\frac{1}{n} \times \sum_{i=1}^{m} x_{i}^{*} \\ \min & f_{2}(\mathbf{x})=1-\frac{1}{n} \times \sum_{i=1}^{m} x_{i}^{*}\end{cases}
$$

A unique characteristic of F22 and F24 is that the sum of all the decision variables of an optimal solution is equal to a constant, and thus, the Pareto front is just one point in the twodimensional objective space. Consequently, the experimental results of $\mathrm{A}-\mathrm{WeB}$ with the 11th weight vector are not included in Fig. 6 for F22 and F24. It is necessary to point out that in the practical implementation of $\mathrm{A}-\mathrm{WeB}$, the probability of $w_{1}=\cdots=w_{n}$ is extremely low due to the randomization.

As shown in Fig. 6, A-WeB performs similarly for the different fixed weight vectors and the hypervolume values move in a small range. Therefore, we can conclude that the performance of $\mathrm{A}-\mathrm{WeB}$ is robust with regard to the change of the weight vector.

\section{B. On the Scalability of $A-W e B$}

To better comprehend the performance of $\mathrm{A}-\mathrm{WeB}$, its scalability is analyzed in this subsection. F05 is selected as a test instance because the number of optimal solutions (NOS) of F05 is scalable with the search range in each dimension. For example, if $\mathcal{S}=[-60,60]^{2}, N O S=76$; if $\mathcal{S}=[-70,70]^{2}, N O S=89 ;$ if $\mathcal{S}=[-80,80]^{2}$, NOS $=$ $101 ;$ if $\mathcal{S}=[-90,90]^{2}, N O S=113$; and if $\mathcal{S}=$ $[-100,100]^{2}, N O S=127$. It can be seen that all the 
TABLE VIII

Comparison of Different Methods on F05 With a LARge Number of Optimal Solutions in Different Decision SPaCes. The Best Result for Each Case Among the Compared Methods is Highlighted in Boldface.

\begin{tabular}{|c|c|c|c|c|c|c|c|c|c|c|}
\hline \multirow{2}{*}{ Method } & \multicolumn{5}{|c|}{$\overline{P R}$} & \multicolumn{5}{|c|}{ 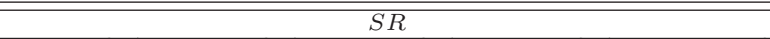 } \\
\hline & {$[-60,60]^{2}$} & {$[-70,70]^{2}$} & {$[-80,80]^{2}$} & {$[-90,90]^{2}$} & {$[-100,100]^{2}$} & {$[-60,60]^{2}$} & {$[-70,70]^{2}$} & {$[-80,80]^{2}$} & {$[-90,90]^{2}$} & {$[-100,100]^{2}$} \\
\hline A-WeB & 0.9921 & 0.9892 & 0.9743 & 0.9122 & 0.9335 & 0.62 & 0.68 & 0.42 & 0.44 & 0.64 \\
\hline A-MONES & 0.9853 & 0.9676 & 0.9343 & 0.7754 & 0.8131 & 0.50 & 0.24 & 0.26 & 0.16 & 0.30 \\
\hline A-MOMMOP & 0.2821 & 0.3611 & 0.4671 & 0.5427 & 0.7874 & 0.00 & 0.00 & 0.00 & 0.00 & 0.30 \\
\hline NCDE & 0.4939 & 0.4335 & 0.3762 & 0.3347 & 0.2885 & 0.00 & 0.00 & 0.00 & 0.00 & 0.00 \\
\hline NSDE & 0.3897 & 0.3204 & 0.2754 & 0.2276 & 0.1957 & 0.00 & 0.00 & 0.00 & 0.00 & 0.00 \\
\hline Rep-SHADE & 0.6700 & 0.6638 & 0.6139 & 0.6234 & 0.6170 & 0.34 & 0.32 & 0.22 & 0.24 & 0.24 \\
\hline Rep-CLPSO & 0.1555 & 0.1342 & 0.0988 & 0.0747 & 0.0688 & 0.00 & 0.00 & 0.00 & 0.00 & 0.00 \\
\hline
\end{tabular}

above cases contain a large number of optimal solutions. Similar to Section V-D, A-WeB is compared with AMONES, A-MOMMOP, NCDE, NSDE, Rep-SHADE, and Rep-CLPSO. For these seven compared methods, $N P=200$ and $M a x_{-} F E s=300,000$. All other parameter settings were kept unchanged.

Table VIII reports the experimental results of different methods on F05. From Table VIII, it is clear that A-WeB is able to consistently provide the best $P R$ and $S R$ values, regardless of the search range in each dimension. Moreover, the $S R$ values provided by $\mathrm{A}-\mathrm{WeB}$ are greater than 0 for all the cases, which means that A-WeB succeeds in finding all the optimal solutions in some independent runs. In contrast, NCDE, NSDE, and Rep-CLPSO cannot achieve any successful run. Therefore, we can conclude that $\mathrm{A}-\mathrm{WeB}$ has better scalability than other compared methods, and has better potential to be applied to NESs with a large number of optimal solutions.

TABLE IX

INFLUENCE OF THE NORMALIZATION OF THE DECISION VARIABLES FOR A-WeB. The Better Result For Each Test Instance Between the COMPaRed METHOdS is Highlighted in BoldFaCE. IN THE LAST

Row, the RESUlts IN THE Form OF $\left(R^{+}, R^{-}, p\right)$ ARE ObTAINED B y the Multiple-Problem Wilcoxon Test.

\begin{tabular}{|c||c|c||c|c|}
\hline \multirow{2}{*}{ Instance } & \multicolumn{2}{|c||}{$P R$} & \multicolumn{2}{c|}{$S R$} \\
\cline { 2 - 5 } & A-WeB & A-WeB-1 & A-WeB & A-WeB-1 \\
\hline F02 & $\mathbf{0 . 6 2 0 0}$ & 0.3800 & $\mathbf{0 . 3 6}$ & 0.10 \\
\hline F04 & $\mathbf{0 . 9 5 7 3}$ & 0.9413 & $\mathbf{0 . 5 8}$ & 0.40 \\
\hline F05 & $\mathbf{1 . 0 0 0 0}$ & 0.8369 & $\mathbf{1 . 0 0}$ & 0.18 \\
\hline F07 & $\mathbf{0 . 9 4 0 0}$ & 0.8875 & $\mathbf{0 . 6 0}$ & 0.34 \\
\hline F08 & 0.4200 & $\mathbf{0 . 7 0 0 0}$ & 0.42 & $\mathbf{0 . 7 0}$ \\
\hline F09 & 0.8371 & 0.8371 & 0.12 & 0.12 \\
\hline F10 & $\mathbf{0 . 8 9 3 3}$ & 0.7867 & $\mathbf{0 . 6 8}$ & 0.56 \\
\hline F12 & $\mathbf{0 . 8 8 8 0}$ & 0.8580 & $\mathbf{0 . 2 8}$ & 0.24 \\
\hline F13 & $\mathbf{0 . 9 7 3 3}$ & 0.8867 & $\mathbf{0 . 7 6}$ & 0.32 \\
\hline F15 & $\mathbf{0 . 6 6 8 8}$ & 0.6450 & 0.00 & 0.00 \\
\hline F16 & 0.9433 & $\mathbf{0 . 9 8 0 0}$ & 0.66 & $\mathbf{0 . 8 8}$ \\
\hline F17 & $\mathbf{0 . 6 2 0 0}$ & 0.5400 & $\mathbf{0 . 2 4}$ & 0.08 \\
\hline F18 & 0.9514 & $\mathbf{0 . 9 7 1 4}$ & 0.70 & $\mathbf{0 . 8 0}$ \\
\hline F19 & 0.9950 & $\mathbf{1 . 0 0 0 0}$ & 0.98 & $\mathbf{1 . 0 0}$ \\
\hline F20 & $\mathbf{1 . 0 0 0 0}$ & 0.9967 & $\mathbf{1 . 0 0}$ & 0.98 \\
\hline F21 & 0.8550 & 0.8550 & 0.14 & 0.14 \\
\hline F26 & $\mathbf{1 . 0 0 0 0}$ & 0.9900 & $\mathbf{1 . 0 0}$ & 0.98 \\
\hline F27 & 0.0933 & $\mathbf{0 . 6 6 3 3}$ & 0.00 & $\mathbf{0 . 0 4}$ \\
\hline F34 & $\mathbf{0 . 8 8 0 0}$ & 0.8600 & $\mathbf{0 . 8 8}$ & 0.86 \\
\hline F36 & $\mathbf{0 . 9 4 0 0}$ & 0.5900 & $\mathbf{0 . 8 8}$ & 0.18 \\
\hline F37 & 0.9320 & $\mathbf{0 . 9 8 8 0}$ & 0.66 & $\mathbf{0 . 9 4}$ \\
\hline F38 & $\mathbf{1 . 0 0 0 0}$ & 0.9950 & $\mathbf{1 . 0 0}$ & 0.98 \\
\hline Average & $\mathbf{0 . 8 3 6 7}$ & 0.8268 & $\mathbf{0 . 5 8 8 2}$ & 0.4918 \\
\hline Wilcoxon test & $(170.5,82.5,1.61 \mathrm{E}-01)$ & $(168.5,84.5,1.52 \mathrm{E}-01)$ \\
\hline & & & \multicolumn{2}{|c}{} \\
\hline \hline & \multicolumn{2}{|c|}{} & & \\
\hline & & &
\end{tabular}

\section{On the Normalization of the Decision Variables}

A-WeB uses a linearly weighted sum of all the decision variables for its location function as shown in Equation (13).
This subsection studies the effect of normalization of the decision variables. For this purpose, the location function in Equation (13) is modified as

$$
\begin{cases}\min & \alpha_{1}(\mathbf{x})=\frac{\sum_{i=1}^{n} w_{i} \times x_{i}^{\prime}}{\sum_{i=1}^{n} w_{i}} \\ \min & \alpha_{2}(\mathbf{x})=1-\frac{\sum_{i=1}^{n} w_{i} \times x_{i}^{\prime}}{\sum_{i=1}^{n} w_{i}}\end{cases}
$$

where $x_{i}^{\prime}=\frac{x_{i}-\underline{x}_{i}}{\bar{x}_{i}-x_{i}}, i=1, \ldots, n$. In this way, $x_{i}^{\prime}$ is normalized in $[0,1]$.

With the modified location function, the resultant $\mathrm{A}-\mathrm{WeB}$ variant is referred to as A-WeB-1. Table IX shows the performance of A-WeB and A-WeB-1 on test instances F01-F21 and F26-F38, where the experimental results are omitted when both of them achieve $P R=1.0$ and $S R=1.0$ for a NES. As can be seen, in contrast to A-WeB-1, A-Web loses on six out of 22 test instances, while gets better results on 14 test instances with respect to $P R$. For $S R$, A-WeB loses on six test instances, yet wins on 13 test instances. In general, A-WeB provides better $P R$ and $S R$ values on average, and higher $R^{+}$ values than $R^{-}$values for the $P R$ and $S R$ metrics.

From the above analysis, it seems that the performance of A-WeB cannot be substantially improved by normalizing the decision variables in the location function. This topic still needs a thorough study in the future.

TABLE X

Results Obtained By the Multiple-Problem Wilcoxon Test for A-WeB with Different Historical Memory Size $H$ on F01-F21.

\begin{tabular}{|l|r|r|c|r|c|c|}
\hline \hline \multirow{2}{*}{$H=100 \mathrm{VS}$} & \multicolumn{3}{|c|}{$P R$} & \multicolumn{3}{c|}{$S R$} \\
\cline { 2 - 7 } & $R^{+}$ & $R^{-}$ & $p$-value & $R^{+}$ & $R^{-}$ & $p$-value \\
\hline$H=5$ & 181.5 & 49.5 & $\mathbf{1 . 2 1 E - 0 2}$ & 169.5 & 61.5 & $6.21 \mathrm{E}-02$ \\
\hline$H=10$ & 175.5 & 55.5 & $\mathbf{3 . 6 8 E - 0 2}$ & 160.5 & 70.5 & $9.73 \mathrm{E}-02$ \\
\hline$H=30$ & 145.5 & 85.5 & $\geq 0.2$ & 135.5 & 95.5 & $\geq 0.2$ \\
\hline$H=50$ & 114.5 & 116.5 & $\geq 0.2$ & 125.5 & 105.5 & $\geq 0.2$ \\
\hline$H=200$ & 59.5 & 171.5 & $\geq 0.2$ & 73.0 & 158.0 & $\geq 0.2$ \\
\hline$H=300$ & 82.0 & 149.0 & $\geq 0.2$ & 89.5 & 141.5 & $\geq 0.2$ \\
\hline$H=400$ & 73.5 & 157.5 & $\geq 0.2$ & 77.0 & 154.0 & $\geq 0.2$ \\
\hline$H=500$ & 102.0 & 129.0 & $\geq 0.2$ & 98.5 & 132.5 & $\geq 0.2$ \\
\hline \hline
\end{tabular}

\section{Influence of the Historical Memory Size $H$}

The historical memory size $H$ is one of the parameters in A-WeB. In the previous experiments, the default setting $H=N P=100$ was adopted as in [31]. In this subsection, its influence on the performance of $\mathrm{A}-\mathrm{WeB}$ is investigated empirically. The detailed experimental results on test instances F01-F21 are respectively given in Tables S-R-VI and S-RVII of the supplemental file for $P R$ and $S R$. In addition, the 
statistical test results by the multiple-problem Wilcoxon test are reported in Table $\mathrm{X}$.

From Tables S-R-VI, S-R-VII, and X, A-WeB with $H<$ 100 shows decreased performance against $H=100$. On the other hand, for $H>100$, the performance of $\mathrm{A}-\mathrm{WeB}$ is improved compared with $H=100$. The comparison in Table $\mathrm{X}$ also indicates that there are no significant differences among A-WeB with the values of $H$ changing from 30 to 500 in that all the $p$-values are greater than 0.05. Therefore, AWeB is insensitive to $H$ and the value of $H$ can be chosen from a large range, for example from 30 to 500 .

TABLE XI

Results ObTained By the MUltiple-Problem Wilcoxon TeST For A-WeB and Its Four Variants With Fixed Parameter Settings ON F01-F21.

\begin{tabular}{|c|c|c|c|c|c|c|}
\hline \hline \multirow{2}{*}{ A-WeB VS } & \multicolumn{3}{|c|}{$P R$} & \multicolumn{3}{c|}{$S R$} \\
\cline { 2 - 7 } & $R^{+}$ & $R^{-}$ & $p$-value & $R^{+}$ & $R^{-}$ & $p$-value \\
\hline A-WeB-2 & 213.0 & 18.0 & $\mathbf{2 . 4 1 E - 0 4}$ & 202.0 & 29.0 & $\mathbf{5 . 3 4 E - 0 4}$ \\
\hline A-WeB-3 & 187.5 & 43.5 & $\mathbf{2 . 7 1 E - 0 3}$ & 182.5 & 48.5 & $\mathbf{1 . 8 5 E - 0 2}$ \\
\hline A-WeB-4 & 191.0 & 40.0 & $\mathbf{7 . 1 0 E - 0 3}$ & 183.5 & 47.5 & $\mathbf{9 . 4 4 E - 0 3}$ \\
\hline A-WeB-5 & 154.5 & 76.5 & $1.54 \mathrm{E}-01$ & 139.0 & 92.0 & $\geq 0.2$ \\
\hline \hline
\end{tabular}

TABLE XII

Rankings Obtained by the Friedman Aligned Test for A-WeB AND ITS Four VARIANTS WITH Fixed PARAMETER SETTINGS ON F01-F21. THE BEST AND THE SECOND BEST RESULTS ARE Highlighted in BoldFACE AND ItAlic, Respectively

\begin{tabular}{|r|c|c|}
\hline Method & Ranking $(P R)$ & Ranking $(S R)$ \\
\hline A-WeB & $\mathbf{3 2 . 2 3 8 1}$ & $\mathbf{3 0 . 5 0 0 0}$ \\
\hline A-WeB-2 & 70.6905 & 75.5476 \\
\hline A-WeB-3 & 64.8571 & 59.5000 \\
\hline A-WeB-4 & 48.6667 & 51.6905 \\
\hline A-WeB-5 & 48.5476 & 47.7619 \\
\hline
\end{tabular}

\section{E. Influence of $F$ and $C R$ in $D E$}

In $\mathrm{A}-\mathrm{WeB}$, the scaling factor $F$ and the crossover control parameter $C R$ of $\mathrm{DE}$ are tuned in an adaptive way. To study the influence of the parameter settings, A-WeB is compared with its variants with fixed $F$ and $C R$. For this purpose, four commonly used settings are selected, i.e., $(F, C R)=$ $(0.9,0.1),(0.9,0.9),(0.5,0.3)$, and $(0.5,0.9)$ [21], [32], [46], and their corresponding methods are referred to as A-WeB-2, A-WeB-3, A-WeB-4, and A-WeB-5, respectively. Tables S$\mathrm{R}$-VIII and S-R-IX in the supplemental file summarize the $P R$ and $S R$ values on test instances F01-F21, respectively. The statistical test results obtained by the multiple-problem Wilcoxon test and the Friedman Aligned test are given in Tables XI and XII, respectively.

As shown in Tables S-R-VIII and S-R-IX, A-WeB provides the best average $P R$ and $S R$ values. From Table XI, A-WeB-2, A-WeB-3, and A-WeB-4 suffer from significant performance degradation since all the $p$-values are less than 0.05 when comparing with $\mathrm{A}-\mathrm{WeB}$ in terms of the $P R$ and $S R$ metrics. It is evident from Table XII that A-WeB ranks the first.

The above comparison indicates that A-WeB exhibits superior performance against its variants with the fixed parameter settings, while avoiding a trial-and-error process to choose the proper parameter values.
TABLE XIII

RANKings of the Methods For PARAmeter AdAPtation by the Friedman Aligned Test on F01-F21. The Best and the SECOND Best Results aRe Highlighted in BoldFACE AND ITALIC, RESPECTIVELY.

\begin{tabular}{|r|c|c|}
\hline \hline Method & Ranking $(P R)$ & Ranking $(S R)$ \\
\hline A-WeB & $\mathbf{2 5 . 3 8 1 0}$ & $\mathbf{2 6 . 4 5 2 4}$ \\
\hline jDE-WeB & 39.6429 & 40.3333 \\
\hline JADE-WeB & 30.9762 & 29.2143 \\
\hline
\end{tabular}

\section{F. Effectiveness of the Parameter Adaptation}

A-WeB adapts the parameters $F$ and $C R$ of DE based on SHADE [31]. Note that adaptive parameter adaptation has been actively studied by DE researchers [47]. In this subsection, the adaptive parameter adaptation of SHADE is replaced with that of two state-of-the-art DE variants, i.e., jDE [48] and JADE [32], and the resultant methods are called jDE-WeB and JADE-WeB, respectively. We compared the performance of A-WeB with that of $\mathrm{jDE}-\mathrm{WeB}$ and JADE-WeB. The $P R$ and $S R$ values resulting from the three compared methods are summarized in Tables S-R-X and S-R-XI of the supplemental file on test instances F01-F21, respectively. In addition, Table XIII reports the statistical test results obtained by the Friedman Aligned test.

As shown in Tables S-R-X, S-R-XI, and XIII, A-WeB provides the best average $P R$ and $S R$ values and ranks the first. Thus, A-WeB achieves the best overall performance. However, according to our observation, there are no significant differences between $\mathrm{A}-\mathrm{WeB}$ and the two competitors in terms of the multiple-problem Wilcoxon test in both the $P R$ and $S R$ metrics. Hence, other adaptive parameter adaptation mechanisms are also effective for handling NESs under our framework.

\section{G. Effectiveness of the Mutation Operator}

In $\mathrm{A}-\mathrm{WeB}$, the mutation operator "DE/current/1" in $\mathrm{E}$ quation (17) is applied. In this subsection, we compared it with another classical mutation operator "DE/rand/1". Note that there are also a lot of other mutation operators in the DE literature [49], [50]. We do not conduct comprehensive comparisons between "DE/current/1" and them because it is out of the scope of this paper. The experimental results are provided in Table S-R-XII of the supplemental file for test instances F01-F21. When the two compared methods are both capable of achieving $P R=1.0$ and $S R=1.0$ for a NES, their results are not reported in Table S-R-XII.

As shown in Table S-R-XII, A-WeB with "DE/current/1" provides better results than $\mathrm{A}-\mathrm{WeB}$ with "DE/rand/1" on most test instances both in terms of the $P R$ and $S R$ metrics. AWeB with "DE/current/1" can also obtain better average $P R$ and $S R$ values. With respect to the multiple-problem Wilcoxon test, although the differences are not significant at $\alpha=0.05$, A-WeB with "DE/current/1" still gets higher $R^{+}$values than $R^{-}$values for both the $P R$ and $S R$ metrics. Therefore, AWeB gets great benefit from "DE/current/1" to find multiple optimal solutions of a NES simultaneously in a single run. 


\section{H. Effect of the Distance Comparison Criterion}

For multimodal optimization problems, Wang et al. [21] presented a new distance comparison criterion to avoid a large attraction basin containing too many similar individuals and to make the distribution of the population more appropriate. In this subsection, this distance comparison criterion is also incorporated into $\mathrm{A}-\mathrm{WeB}$ for solving NESs. The corresponding A-WeB variant is called A-WeB-6. In A-WeB-6, an individual $\mathbf{x}_{u}$ is said to be better than another individual $\mathbf{x}_{v}$ if

$\sum_{i=1}^{m}\left|e_{i}\left(\mathbf{x}_{u}\right)\right|<\sum_{i=1}^{m}\left|e_{i}\left(\mathbf{x}_{v}\right)\right| \wedge$ norm_dist $\left(\mathbf{x}_{u}, \mathbf{x}_{v}\right)<\delta$

where norm_dist $\left(\mathbf{x}_{u}, \mathbf{x}_{v}\right)$ denotes the normalized Euclidean distance between $\mathbf{x}_{u}$ and $\mathbf{x}_{v}$, and $\delta$ is a distance threshold which is set to 0.01 [21]. The experimental results are given in Table S-R-XIII of the supplemental file for test instances F01F21. Again, the experimental results of those test instances, for which A-WeB and A-WeB-6 can achieve 100\% PR and $100 \% S R$, are omitted.

From Table S-R-XIII, A-WeB-6 is able to obtain better results on 12 and 11 out of 16 test instances in terms of the $P R$ and $S R$ metrics, respectively. Compared with A-WeB, AWeB-6 also gets better average $P R$ and $S R$ values, and higher $R^{+}$values than $R^{-}$values for both the $P R$ and $S R$ metrics. Therefore, this distance comparison criterion is applicable to further improve the performance of $\mathrm{A}-\mathrm{WeB}$.

\section{CONCLUSIONS AND Future WORK}

This paper has proposed a weighted biobjective transformation technique named WeB to formulate a NES as a biobjective optimization problem, which extends our previous work [3]. WeB attempts to produce a weighted linear combination of all the decision variables in the two objective functions. Thanks to random weights, $\mathrm{WeB}$ is very likely to achieve a one-to-one mapping from the optimal solutions of a NES to different points on the Pareto front of the transformed biobjective optimization problem. Therefore, it can alleviate the risk of losing some optimal solutions with the same values in certain decision variables due to the many-to-one mapping in [3]. Subsequently, we have also suggested an adaptive multiobjective differential evolution named AMODE as the optimization algorithm. By combining WeB with AMODE, a generic framework referred to as $\mathrm{A}-\mathrm{WeB}$, has been proposed for dealing with NESs. It is worth noting that the Pareto front of the transformed biobjective optimization problem is linear. We thus expect that A-Web is able to effectively locate multiple Pareto optimal solutions in a single run. As a result, the optimal solutions of a NES can also be obtained correspondingly.

The performance of $\mathrm{A}-\mathrm{WeB}$ has been extensively tested on 38 test instances which include 21 NESs with known optimal solutions, four NESs with infinitely many optimal solutions, and 13 ill-scaled NESs. Moreover, A-WeB is compared with three multiobjective optimization-based transformation techniques equipped with AMODE as the optimization algorithm and six well-established single-objective optimization-based methods. The empirical studies verify that $\mathrm{A}-\mathrm{WeB}$ has the best overall performance across these three kinds of NESs.
Since there is no prior knowledge about the importance of each decision variable, the weights in $\mathrm{A}-\mathrm{WeB}$ were randomly generated. In the future, we plan to design adaptive/selfadaptive weights by online analysis of the importance of the decision variables according to the properties of NESs at hand. Additionally, developing other advanced optimization algorithms (such as the multioperator-based EAs [46], [51]) for NESs will be another part of our future work.

The C++ source code of A-WeB can be downloaded from Y. Wang's homepage: http://ist.csu.edu.cn/YongWang.htm

\section{ACKNOWLEDGMENTS}

The authors would like to thank Prof. A. Zhou for his constructive comments and suggestions. They would also like to thank Prof. P. N. Suganthan for providing the source codes of NCDE, NSDE, and LIPS.

\section{REFERENCES}

[1] D. Mehta and C. Grosan, "A collection of challenging optimization problems in science, engineering and economics," in 2015 IEEE Congres on Evolutionary Computation (CEC), May 2015, pp. 2697-2704.

[2] C. Karr, B. Weck, and L. Freeman, "Solutions to systems of nonlinear equations via a genetic algorithm," Engineering Applications of Artificial Intelligence, vol. 11, no. 3, pp. 369-375, 1998.

[3] W. Song, Y. Wang, H.-X. Li, and Z. Cai, "Locating multiple optimal solutions of nonlinear equation systems based on multiobjective optimization," IEEE Trans. on Evol. Comput., vol. 19, no. 3, pp. 414-431, June 2015 .

[4] R. Brits, A. Engelbrecht, and F. van den Bergh, "Solving systems of unconstrained equations using particle swarm optimization," in 2002 IEEE International Conference on Systems, Man and Cybernetics, vol. 3, 2002, pp. 6-9.

[5] Y. Mo, H. Liu, and Q. Wang, "Conjugate direction particle swarm optimization solving systems of nonlinear equations," Computers \& Mathematics with Applications, vol. 57, no. 11-12, pp. 1877-1882, 2009.

[6] A. Noriega, J. Cortizo, E. Rodriguez, R. Vijande, and J. Sierra, "A new method to approximate the field of movements of 1-DOF linkages with lower-pairs," Meccanica, vol. 45, no. 5, pp. 681-692, 2010.

[7] M. Jaberipour, E. Khorram, and B. Karimi, "Particle swarm algorithm for solving systems of nonlinear equations," Computers \& Mathematics with Applications, vol. 62, no. 2, pp. 566-576, 2011.

[8] C. Voglis, K. Parsopoulos, and I. Lagaris, "Particle swarm optimization with deliberate loss of information," Soft Computing, vol. 16, no. 8, pp 1373-1392, 2012

[9] P. S. Mhetre, "Genetic algorithm for linear and nonlinear equation," International Journal of Advanced Engineering Technology, vol. 3, no. 2, pp. 114-118, 2012.

[10] H. A. e Oliveira Jr. and A. Petraglia, "Solving nonlinear systems of functional equations with fuzzy adaptive simulated annealing," Applied Soft Computing, vol. 13, no. 11, pp. 4349-4357, 2013.

[11] O. E. Turgut, M. S. Turgut, and M. T. Coban, "Chaotic quantum behaved particle swarm optimization algorithm for solving nonlinear system of equations," Computers \& Mathematics with Applications, vol. 68, no. 4, pp. 508-530, 2014

[12] A. F. Kuri-Morales, "Solution of simultaneous non-linear equations using genetic algorithms," WSEAS Transactions on Systems, vol. 2, no. 1 , pp. 44-51, 2003.

[13] A. Pourrajabian, R. Ebrahimi, M. Mirzaei, and M. Shams, "Applying genetic algorithms for solving nonlinear algebraic equations," Applied Mathematics and Computation, vol. 219, no. 24, pp. 11483-11494, 2013

[14] C. Grosan and A. Abraham, "A new approach for solving nonlinear equations systems," IEEE Transactions on Systems, Man and Cybernetics, Part A: Systems and Humans, vol. 38, no. 3, pp. 698-714, May 2008.

[15] S. Qin, S. Zeng, W. Dong, and X. Li, "Nonlinear equation systems solved by many-objective hype," in 2015 IEEE Congress on Evolutionary Computation, 2015, pp. 2691-2696. 
[16] M. J. Hirsch, P. M. Pardalos, and M. G. Resende, "Solving systems of nonlinear equations with continuous GRASP,' Nonlinear Analysis: Real World Applications, vol. 10, no. 4, pp. 2000-2006, 2009.

[17] E. Pourjafari and H. Mojallali, "Solving nonlinear equations systems with a new approach based on invasive weed optimization algorithm and clustering," Swarm and Evolutionary Computation, vol. 4, no. 0 , pp. 33-43, 2012

[18] N. Henderson, W. F. Sacco, and G. M. Platt, "Finding more than one root of nonlinear equations via a polarization technique: An application to double retrograde vaporization," Chemical Engineering Research and Design, vol. 88, no. 5C6, pp. 551-561, 2010.

[19] S. Das, S. Maity, B.-Y. Qu, and P. N. Suganthan, "Real-parameter evolutionary multimodal optimization - A survey of the state-of-theart," Swarm and Evolutionary Computation, vol. 1, no. 2, pp. 71-88, 2011.

[20] H. Ishibuchi, N. Akedo, and Y. Nojima, "Behavior of multiobjective evolutionary algorithms on many-objective knapsack problems," IEEE Trans. on Evol. Comput., vol. 19, no. 2, pp. 264-283, 2015.

[21] Y. Wang, H.-X. Li, G. G. Yen, and W. Song, "MOMMOP: Multiobjective optimization for locating multiple optimal solutions of multimodal optimization problems," IEEE Transactions on Cybernetics, vol. 45, no. 4, pp. 830-843, April 2015

[22] A. Basak, S. Das, and K. Tan, "Multimodal optimization using a biobjective differential evolution algorithm enhanced with mean distance-based selection," IEEE Trans. on Evol. Comput., vol. 17, no. 5, pp. 666-685, 2013.

[23] J. Yao, N. Kharma, and P. Grogono, "Bi-objective multipopulation genetic algorithm for multimodal function optimization," IEEE Trans. on Evol. Comput., vol. 14, no. 1, pp. 80-102, Feb 2010.

[24] K. Deb and A. Saha, "Multimodal optimization using a bi-objective evolutionary algorithm," Evolutionary Computation, vol. 20, no. 1, pp. 27-62, 2012.

[25] S. Bandaru and K. Deb, "A parameterless-niching-assisted bi-objective approach to multimodal optimization," in 2013 IEEE Congress on Evolutionary Computation (CEC), 2013, pp. 95-102.

[26] K. Deb, A. Pratap, S. Agarwal, and T. Meyarivan, "A fast and elitist multiobjective genetic algorithm: NSGA-II," IEEE Trans. on Evol. Comput., vol. 6, no. 2, pp. 182-197, 2002.

[27] T. Robič and B. Filipič, "DEMO: Differential evolution for multiobjective optimization," in Evolutionary Multi-Criterion Optimization, ser. LNCS, C. A. Coello Coello, A. Hernández Aguirre, and E. Zitzler, Eds., 2005, vol. 3410, pp. 520-533.

[28] R. Storn and K. Price, "Differential evolution-A simple and efficient heuristic for global optimization over continuous spaces," J. of Global Optim., vol. 11, no. 4, pp. 341-359, Dec 1997.

[29] R. Gämperle, S. Müler, and P. Koumoutsakos, "A parameter study for differential evolution," in Proc. WSEAS Int. Conf. Advances Intell. Syst. Fuzzy Syst., Evol. Comput., 2002, pp. 293-298.

[30] Y. Wang, Z. Cai, and Q. Zhang, "Differential evolution with composite trial vector generation strategies and control parameters," IEEE Trans. on Evol. Comput., vol. 15, no. 1, pp. 55-66, 2011.

[31] R. Tanabe and A. Fukunaga, "Success-history based parameter adaptation for differential evolution," in 2013 IEEE Congress on Evolutionary Computation (CEC), 2013, pp. 71-78

[32] J. Zhang and A. C. Sanderson, "JADE: Adaptive differential evolution with optional external archive," IEEE Trans. on Evol. Comput., vol. 13 , no. 5, pp. 945-958, Oct 2009.

[33] H. Li and Q. Zhang, "Multiobjective optimization problems with complicated pareto sets, MOEA/D and NSGA-II," IEEE Trans. on Evol. Comput., vol. 13, no. 2, pp. 284-302, 2009.

[34] K. Li, A. Fialho, S. Kwong, and Q. Zhang, "Adaptive operator selection with bandits for a multiobjective evolutionary algorithm based on decomposition," IEEE Trans. on Evol. Comput., vol. 18, no. 1, pp. 114 130, 2014.

[35] R. B. Kearfott, "Some tests of generalized bisection," ACM Trans. Math. Softw., vol. 13, no. 3, pp. 197-220, 1987.

[36] K. Meintjes and A. P. Morgan, "Chemical equilibrium systems as numerical test problems," ACM Trans. Math. Softw., vol. 16, no. 2, pp. $143-151,1990$

[37] W. Sacco and N. Henderson, "Finding all solutions of nonlinear systems using a hybrid metaheuristic with fuzzy clustering means," Applied Soft Computing, vol. 11, no. 8, pp. 5424-5432, 2011.

[38] X. Li, A. Engelbrecht, and M. G. Epitropakis, "Benchmark functions for CEC2013 special session and competition on niching methods for multimodal function optimization," Evolutionary Computation and Machine Learning Group, RMIT University, Australia, Tech. Rep., 2013.
[39] K. Deb, Multi-Objective Optimization Using Evolutionary Algorithms. New York: Wiley, 2001.

[40] B. Qu, P. Suganthan, and J. Liang, "Differential evolution with neighborhood mutation for multimodal optimization," IEEE Trans. on Evol. Comput., vol. 16, no. 5, pp. 601-614, Oct 2012.

[41] B. Qu, P. Suganthan, and S. Das, "A distance-based locally informed particle swarm model for multimodal optimization," IEEE Trans. on Evol. Comput., vol. 17, no. 3, pp. 387-402, June 2013.

[42] X. Li, "Niching without niching parameters: Particle swarm optimization using a ring topology," IEEE Trans. on Evol. Comput., vol. 14, no. 1, pp. 150-169, Feb 2010

[43] J. J. Liang, A. K. Qin, P. N. Suganthan, and S. Baskar, "Comprehensive learning particle swarm optimizer for global optimization of multimodal functions," IEEE Trans. on Evol. Comput., vol. 10, no. 3, pp. 281-295, 2006.

[44] S. García, D. Molina, M. Lozano, and F. Herrera, "A study on the use of non-parametric tests for analyzing the evolutionary algorithms' behaviour: A case study on the CEC'2005 special session on real parameter optimization," Journal of Heuristics, vol. 15, no. 6, pp. 617644, 2009.

[45] J. Alcalá-Fdez, L. Sánchez, and S. García, "KEEL: A software too to assess evolutionary algorithms to data mining problems," 2015. [Online]. Available: http://www.keel.es/

[46] A. K. Qin, V. L. Huang, and P. N. Suganthan, "Differential evolution algorithm with strategy adaptation for global numerical optimization,' IEEE Trans. on Evol. Comput., vol. 13, no. 2, pp. 398-417, Apr 2009.

[47] M. Drozdik, H. Aguirre, Y. Akimoto, and K. Tanaka, "Comparison of parameter control mechanisms in multi-objective differential evolution," in Learning and Intelligent Optimization, ser. Lecture Notes in Computer Science, C. Dhaenens, L. Jourdan, and M.-E. Marmion, Eds., 2015, vol. 8994.

[48] J. Brest, S. Greiner, B. Bošković, M. Mernik, and V. Žumer, "Selfadapting control parameters in differential evolution: A comparative study on numerical benchmark problems," IEEE Trans. on Evol. Comput., vol. 10, no. 6, pp. 646-657, Dec 2006.

[49] K. Price, R. Storn, and J. Lampinen, Differential Evolution: A Practical Approach to Global Optimization. Berlin: Springer-Verlag, 2005.

[50] J. Zhang and A. C. Sanderson, Adaptive Differential Evolution: A Robust Approach to Multimodal Problem Optimization. Berlin: SpringerVerlag, 2009.

[51] W. Gong, A. Zhou, and Z. Cai, "A multioperator search strategy based on cheap surrogate models for evolutionary optimization," IEEE Trans. on Evol. Comput., vol. 19, no. 5, pp. 746-758, 2015.

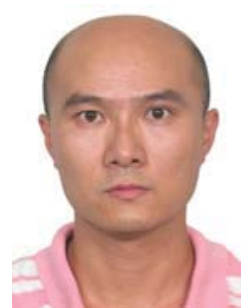

Wenyin Gong received the B.Eng., M.Eng., and $\mathrm{PhD}$ degrees in computer science from China $\mathrm{U}$ niversity of Geosciences, Wuhan, China, in 2004 2007, and 2010, respectively.

$\mathrm{He}$ is a Professor with School of Computer Science, China University of Geosciences, Wuhan, China. His research interests include evolutionary algorithms, evolutionary optimization, and their applications. He has published over 50 research papers in journals and international conferences.

He served as a referee for over 20 international journals, such as IEEE TRANSACTIONS ON EVOLUTIONARY COMPUTATION, IEEE TRANSACTIONS ON CYBERNETICS, IEEE Computational Intelligence Magazine, ACM Transactions on Intelligent Systems and Technology, Information Sciences, European Journal of Operational Research, Applied Soft Computing, International Journal of Hydrogen Energy, and so on. 


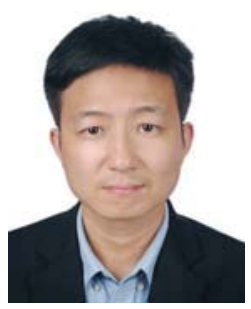

intelligence.

Yong Wang (M'08) received the B.S. degree in automation from the Wuhan Institute of Technology, Wuhan, China, in 2003, and the M.S. degree in pattern recognition and intelligent systems and the Ph.D. degree in control science and engineering both from the Central South University (CSU), Changsha, China, in 2006 and 2011, respectively. He is currently an Associate Professor with the School of Information Science and Engineering, CSU. His current research interests include the theory, algorithm design, and applications of computational

Dr. Wang is a member of the IEEE CIS Task Force on Nature-Inspired Constrained Optimization and the IEEE CIS Task Force on Differential Evolution. He was a reviewer of 50+ international journals and a PC member of $40+$ international conferences. He was awarded the Hong Kong Scholar by the Mainland - Hong Kong Joint Postdoctoral Fellows Program, China, in 2013, the Excellent Doctoral Dissertation by Hunan Province, China, in 2013, the New Century Excellent Talents in University by the Ministry of Education, China, in 2013, the 2015 IEEE Computational Intelligence Society Outstanding PhD Dissertation Award, the Hunan Provincial Natural Science Fund for Distinguished Young Scholars, in 2016, and the EU Horizon 2020 Marie Sklodowska-Curie Fellowship, in 2016. He is currently serving as an associate editor for the Swarm and Evolutionary Computation.

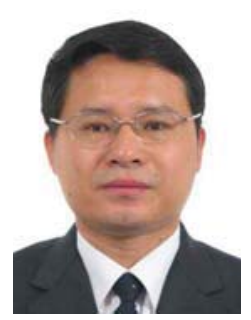

Zhihua Cai received the Bsc degree from Wuhan University, China, in 1986, the Msc degree from Beijing University of Technology, China, in 1992 and the $\mathrm{PhD}$ degree from China University of Geosciences, in 2003.

$\mathrm{He}$ is currently a faculty member at School of Computer Science, China University of Geosciences, Wuhan, China. His main research areas include data mining, machine learning, evolutionary computation, and their applications. He has published over 100 research papers.

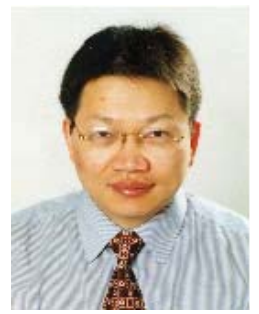

Shengxiang Yang (M'00-SM'14) received the B.Sc and M.Sc. degrees in automatic control and the $\mathrm{Ph} . \mathrm{D}$. degree in systems engineering from Northeastern University, Shenyang, China in 1993, 1996, and 1999, respectively.

$\mathrm{He}$ is currently a Professor in Computational Intelligence and Director of the Centre for Computational Intelligence, School of Computer Science and Informatics, De Montfort University, Leicester, U.K. He has over 220 publications. His current research interests include evolutionary computation, swarm intelligence, computational intelligence in dynamic and uncertain environments, artificial neural networks for scheduling, and relevant real-world applications. He serves as an Associate Editor or Editorial Board Member of 8 international journals, such as the IEEE Transactions on Cybernetics, Information Sciences, Evolutionary Computation, Neurocomputing, and Soft Computing. 


\section{Supplemental file for "A Weighted Biobjective Transformation Technique for Locating Multiple Optimal Solutions of Nonlinear Equation Systems"}

Wenyin Gong, Yong Wang, Member, IEEE, Zhihua Cai, and Shengxiang Yang, Senior Member, IEEE

\section{S-I. ThirTy-eIght Test Instances}

1) F01:

$$
\left\{\begin{array}{l}
x_{1}^{2}+x_{2}^{2}-1=0 \\
x_{1}-x_{2}=0
\end{array}\right.
$$

where $x_{i} \in[-1,1], i=1, \cdots, n$, and $n=2$. It has two optimal solutions: $(-0.707107,-0.707107)$ and $(0.707107$, 0.707107) [1].

2) F02:

$$
\left\{\begin{array}{l}
\sum_{i=1}^{n} x_{i}^{2}-1=0 \\
\left|x_{1}-x_{2}\right|+\sum_{i=3}^{n} x_{i}^{2}=0
\end{array}\right.
$$

where $x_{i} \in[-1,1], i=1, \cdots, n$, and $n=20$. It has two optimal solutions: $(-0.707107,-0.707107,0, \cdots, 0)$ and $(0.707107,0.707107,0, \cdots, 0)[1]$.

3) F03:

$$
\left\{\begin{array}{l}
x_{1}-\sin \left(5 \pi x_{2}\right)=0 \\
x_{1}-x_{2}=0
\end{array}\right.
$$

where $x_{i} \in[-1,1], i=1, \cdots, n$, and $n=2$. It has 11 optimal solutions as shown in Table S-I [1].

TABLE S-I

THE OPTIMAL SOLUTIONS OF F03

\begin{tabular}{|r|r|}
\hline$x_{1}$ & $x_{2}$ \\
\hline-0.924840 & -0.924840 \\
-0.866760 & -0.866760 \\
-0.562010 & -0.562010 \\
-0.428168 & -0.428168 \\
-0.187960 & -0.187960 \\
0.000000 & 0.000000 \\
0.187960 & 0.187960 \\
0.428168 & 0.428168 \\
0.562010 & 0.562010 \\
0.866760 & 0.866760 \\
0.924840 & 0.924840 \\
\hline
\end{tabular}

4) F04:

$$
\left\{\begin{array}{l}
x_{1}-\cos \left(4 \pi x_{2}\right)=0 \\
x_{1}^{2}+x_{2}^{2}-1=0
\end{array}\right.
$$

where $x_{i} \in[-1,1], i=1, \cdots, n$, and $n=2$. It has 15 optimal solutions as shown in Table S-II [1].

W. Gong and Z. Cai are with the School of Computer Science, China University of Geosciences, Wuhan 430074, China. (Email: wygong@cug.edu.cn; zhcai@cug.edu.cn)

Y. Wang is with the School of Information Science and Engineering, Central South University, Changsha 410083, China, and also with the Centre for Computational Intelligence (CCI), School of Computer Science and Informatics, De Montfort University, Leicester LE1 9BH, UK. (Email: ywang@ @ csu.edu.cn)

S. Yang is with the Centre for Computational Intelligence (CCI), School of Computer Science and Informatics, De Montfort University, Leicester LE1 9BH, UK. (Email: syang@dmu.ac.uk)
TABLE S-II

THE OPTIMAL SOLUTIONS OF F04

\begin{tabular}{|r|r|}
\hline$x_{1}$ & $x_{2}$ \\
\hline 0.416408 & -0.909178 \\
-0.561364 & -0.827569 \\
-0.724322 & -0.689462 \\
0.837812 & -0.545959 \\
0.886984 & 0.461799 \\
-0.962322 & -0.271914 \\
-0.972855 & -0.231415 \\
1.000000 & 0.000000 \\
-0.972855 & 0.231416 \\
-0.962322 & 0.271914 \\
0.886984 & 0.461799 \\
0.837812 & 0.545959 \\
-0.724322 & 0.689462 \\
-0.561364 & 0.827569 \\
0.416408 & 0.909178 \\
\hline
\end{tabular}

5) F05:

$$
\left\{\begin{array}{l}
\cos \left(2 x_{1}\right)-\cos \left(2 x_{2}\right)-0.4=0 \\
2\left(x_{2}-x_{1}\right)+\sin \left(2 x_{2}\right)-\sin \left(2 x_{1}\right)-1.2=0
\end{array}\right.
$$

where $x_{i} \in[-10,10], i=1, \cdots, n$, and $n=2$. It has 13 optimal solutions as shown in Table S-III [2].

TABLE S-III

THE OPTIMAL SOLUTIONS OF F05

\begin{tabular}{|r|r|}
\hline$x_{1}$ & $x_{2}$ \\
\hline-9.268258 & -8.931402 \\
-8.744542 & -7.164787 \\
-6.126665 & -5.789809 \\
-5.602950 & -4.023195 \\
-2.985073 & -2.648216 \\
-2.461357 & -0.881602 \\
0.156520 & 0.493376 \\
0.680236 & 2.259991 \\
3.298113 & 3.634969 \\
3.821828 & 5.401583 \\
6.439705 & 6.776562 \\
6.963421 & 8.543176 \\
9.581298 & 9.918154 \\
\hline
\end{tabular}

6) F06:

$$
\left\{\begin{array}{l}
x_{1}-0.25428722-0.18324757 x_{4} x_{3} x_{9}=0 \\
x_{2}-0.37842197-0.16275449 x_{1} x_{10} x_{6}=0 \\
x_{3}-0.27162577-0.16955071 x_{1} x_{2} x_{10}=0 \\
x_{4}-0.19807914-0.15585316 x_{7} x_{1} x_{6}=0 \\
x_{5}-0.44166728-0.19950920 x_{7} x_{6} x_{3}=0 \\
x_{6}-0.14654113-0.18922793 x_{8} x_{5} x_{10}=0 \\
x_{7}-0.42937161-0.21180486 x_{2} x_{5} x_{8}=0 \\
x_{8}-0.07056438-0.17081208 x_{1} x_{7} x_{6}=0 \\
x_{9}-0.34504906-0.19612740 x_{10} x_{6} x_{8}=0 \\
x_{10}-0.42651102-0.21466544 x_{4} x_{8} x_{1}=0
\end{array}\right.
$$

where $x_{i} \in[-2,2], i=1, \cdots, n$, and $n=10$. It has one optimal solution: $(0.257833,0.381097,0.278745$, 
$0.200669,0.445251,0.149184,0.432010,0.073403,0.345967$, $0.427326)[2]$.

7) F07:

$$
\left\{\begin{array}{l}
100\left(x_{1}-0.25\right)=0 \\
100\left(x_{1} \sin \left(4 \pi x_{2}^{2}\right)+0.75 x_{1}-0.25\right)=0
\end{array}\right.
$$

where $x_{i} \in[-1,1], i=1, \cdots, n$, and $n=2$. It has eight optimal solutions as shown in Table S-IV.

TABLE S-IV

THE OPTIMAL SOLUTIONS OF F07

\begin{tabular}{|r|r|}
\hline$x_{1}$ & $x_{2}$ \\
\hline 0.250000 & -0.854337 \\
0.250000 & -0.721185 \\
0.250000 & -0.479471 \\
0.250000 & -0.141801 \\
0.250000 & 0.141801 \\
0.250000 & 0.479471 \\
0.250000 & 0.721185 \\
0.250000 & 0.854337 \\
\hline
\end{tabular}

8) F08:

$$
\left\{\begin{array}{l}
3.0-x_{1} x_{3}^{2}=0 \\
x_{3} \sin \left(\pi / x_{2}\right)-x_{3}-x_{4}=0 \\
-x_{2} x_{3} \exp \left(1.0-x_{1} x_{3}\right)+0.2707=0 \\
2 x_{1}^{2} x_{3}-x_{2}^{4} x_{3}-x_{2}=0
\end{array}\right.
$$

where $x_{i} \in[0,5], i=1, \cdots, n$, and $n=4$. It has one optimal solution: $(3,2,1,0)[3]$.

9) F09:

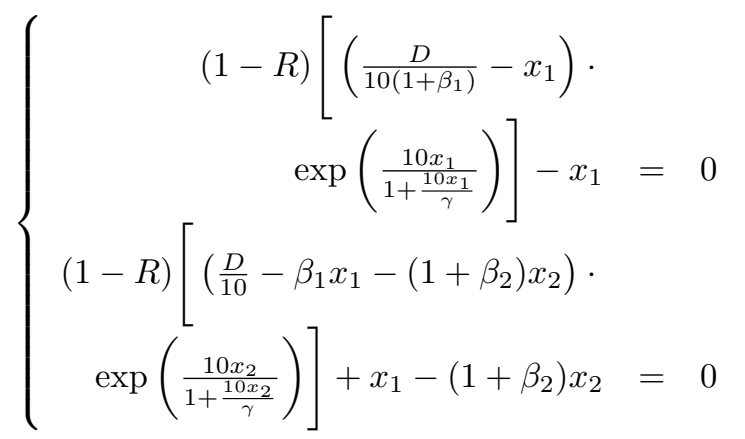

where $x_{i} \in[0,1], i=1, \cdots, n, n=2, R=0.96, D=22$, $\gamma=1000$, and $\beta_{1}=\beta_{2}=2$. It has seven optimal solutions as shown in Table $\mathrm{S}-\mathrm{V}$ [3], [4], [5], [6].

TABLE S-V

THE OPTIMAL SOLUTIONS OF F09

\begin{tabular}{|r|r|}
\hline$x_{1}$ & $x_{2}$ \\
\hline 0.042100 & 0.061813 \\
0.042100 & 0.268723 \\
0.266600 & 0.178430 \\
0.266600 & 0.327267 \\
0.266600 & 0.461111 \\
0.042318 & 0.686779 \\
0.719074 & 0.244197 \\
\hline
\end{tabular}

10) F10:

$$
\left\{\begin{array}{l}
2 x_{1}+x_{2}+x_{3}+x_{4}+x_{5}-6.0=0 \\
x_{1}+2 x_{2}+x_{3}+x_{4}+x_{5}-6.0=0 \\
x_{1}+x_{2}+2 x_{3}+x_{4}+x_{5}-6.0=0 \\
x_{1}+x_{2}+x_{3}+2 x_{4}+x_{5}-6.0=0 \\
x_{1} x_{2} x_{3} x_{4} x_{5}-1.0=0
\end{array}\right.
$$

where $x_{i} \in[-10,10], i=1, \cdots, n$, and $n=5$. It has three optimal solutions: $(1,1,1,1,1),(0.916355,0.916355$, $0.916355,0.916355,1.418227)$, and $(-0.579043,-0.579043$, $-0.579043,-0.579043,8.895215)$ [7], [8].

11) F11:

$$
\left\{\begin{array}{l}
x_{1}+x_{2}^{4} x_{4} x_{6} / 4+0.75=0 \\
x_{2}+0.405 \exp \left(1+x_{1} x_{2}\right)-1.405=0 \\
x_{3}-x_{4} x_{6} / 2+1.5=0 \\
x_{4}-0.605 \exp \left(1-x_{3}^{2}\right)-0.395=0 \\
x_{5}-x_{2} x_{6} / 2+1.5=0 \\
x_{6}-x_{1} x_{5}=0
\end{array}\right.
$$

where $x_{i} \in[-1,1], i=1, \cdots, n$, and $n=6$. It has one optimal solution: $(-1,1,-1,1,-1,1)$ [8], [9].

12) F12:

$$
\left\{\begin{array}{l}
\sin \left(x_{1}^{3}\right)-3 x_{1} x_{2}^{2}-1=0 \\
\cos \left(3 x_{1}^{2} x_{2}\right)-\left|x_{2}^{3}\right|+1=0
\end{array}\right.
$$

where $x_{i} \in[-2,2], i=1, \cdots, n, n=2$. It has 10 optimal solutions as shown in Table S-VI. This function is modified from [10].

TABLE S-VI

THE OPTIMAL SOLUTIONS OF F12

\begin{tabular}{|r|r|}
\hline$x_{1}$ & $x_{2}$ \\
\hline-1.810885 & -0.349092 \\
-1.810885 & 0.349092 \\
-1.502221 & -0.409077 \\
-1.502221 & 0.409077 \\
-1.791302 & 0.301926 \\
-1.791302 & -0.301926 \\
-0.947268 & 0.785020 \\
-0.947268 & -0.785020 \\
-0.213057 & 1.256845 \\
-0.213057 & -1.256845 \\
\hline
\end{tabular}

13) F13:

$$
\left\{\begin{array}{l}
4 x_{1}^{3}+4 x_{1} x_{2}+2 x_{2}^{2}-42 x_{1}-14=0 \\
4 x_{2}^{3}+2 x_{1}^{2}+4 x_{1} x_{2}-26 x_{2}-22=0
\end{array}\right.
$$

where $x_{i} \in[-5,5], i=1, \cdots, n$, and $n=2$. It has nine optimal solutions as shown in Table S-VII [11], [12].

TABLE S-VII

THE OPTIMAL SOLUTIONS OF F13

\begin{tabular}{|r|r|}
\hline$x_{1}$ & $x_{2}$ \\
\hline-0.127961 & -1.953715 \\
-0.270845 & -0.923039 \\
0.086678 & 2.884255 \\
3.385154 & 0.073852 \\
3.584428 & -1.848127 \\
3.000000 & 2.000000 \\
-3.779310 & -3.283186 \\
-3.073026 & -0.081353 \\
-2.805118 & 3.131313 \\
\hline
\end{tabular}

14) F14:

$$
\left\{\begin{array}{l}
-\sin (x 1) \cos (x 2)-2 \cos (x 1) \sin (x 2)=0 \\
-\cos (x 1) \sin (x 2)-2 \sin (x 1) \cos (x 2)=0
\end{array}\right.
$$

where $x_{i} \in[0,2 \pi], i=1, \cdots, n$, and $n=2$. It has 13 optimal solutions as shown in Table S-VIII [5], [11]. 
TABLE S-VIII

THE OPTIMAL SOLUTIONS OF F14

\begin{tabular}{|r|r|}
\hline$x_{1}$ & $x_{2}$ \\
\hline 0.000000 & 0.000000 \\
3.141593 & 0.000000 \\
1.570796 & 1.570796 \\
6.283185 & 0.000000 \\
0.000000 & 3.141593 \\
4.712389 & 1.570796 \\
3.141593 & 3.141593 \\
1.570796 & 4.712389 \\
6.283185 & 3.141593 \\
0.000000 & 6.283185 \\
4.712389 & 4.712389 \\
3.141593 & 6.283185 \\
6.283185 & 6.283185 \\
\hline
\end{tabular}

15) F15:

$$
\left\{\begin{aligned}
x_{1}^{2}+x_{2}^{2}-1.0 & =0 \\
x_{3}^{2}+x_{4}^{2}-1.0 & =0 \\
x_{5}^{2}+x_{6}^{2}-1.0 & =0 \\
x_{7}^{2}+x_{8}^{2}-1.0 & =0 \\
4.731 \cdot 10^{-3} x_{1} x_{3}-0.3578 x_{2} x_{3}-0.1238 x_{1}+x_{7} & \\
-1.637 \cdot 10^{-3} x_{2}-0.9338 x_{4}-0.3571 & =0 \\
0.2238 x_{1} x_{3}+0.7623 x_{2} x_{3}+0.2638 x_{1}-x_{7} & \\
-0.07745 x_{2}-0.6734 x_{4}-0.6022 & =0 \\
x_{6} x_{8}+0.3578 x_{1}+4.731 \cdot 10^{-3} x_{2} & =0 \\
-0.7623 x_{1}+0.2238 x_{2}+0.3461 & =0
\end{aligned}\right.
$$

where $x_{i} \in[-1,1], i=1, \cdots, n$, and $n=8$. It has 16 optimal solutions as shown in Table S-IX [7], [11], [12].

TABLE S-IX

THE OPTIMAL SOLUTIONS OF F15

\begin{tabular}{|r|r|r|r|r|r|r|r|}
\hline$x_{1}$ & $x_{2}$ & $x_{3}$ & $x_{4}$ & $x_{5}$ & $x_{6}$ & $x_{7}$ & $x_{8}$ \\
\hline 0.1644 & -0.9864 & -0.9471 & -0.3210 & -0.9982 & -0.0594 & 0.4110 & 0.9116 \\
0.1644 & -0.9864 & -0.9471 & -0.3210 & -0.9982 & 0.0594 & 0.4110 & -0.9116 \\
0.1644 & -0.9864 & -0.9471 & -0.3210 & 0.9982 & -0.0594 & 0.4110 & 0.9116 \\
0.1644 & -0.9864 & -0.9471 & -0.3210 & 0.9982 & 0.0594 & 0.4110 & -0.9116 \\
0.1644 & -0.9864 & 0.7185 & -0.6956 & -0.9980 & -0.0638 & -0.5278 & 0.8494 \\
0.1644 & -0.9864 & 0.7185 & -0.6956 & -0.9980 & 0.0638 & -0.5278 & -0.8494 \\
0.1644 & -0.9864 & 0.7185 & -0.6956 & 0.9980 & -0.0638 & -0.5278 & 0.8494 \\
0.1644 & -0.9864 & 0.7185 & -0.6956 & 0.9980 & 0.0638 & -0.5278 & -0.8494 \\
0.6716 & 0.7410 & -0.6516 & -0.7586 & -0.9625 & -0.2711 & -0.4376 & 0.8992 \\
0.6716 & 0.7410 & -0.6516 & -0.7586 & -0.9625 & 0.2711 & -0.4376 & -0.8992 \\
0.6716 & 0.7410 & -0.6516 & -0.7586 & 0.9625 & -0.2711 & -0.4376 & 0.8992 \\
0.6716 & 0.7410 & -0.6516 & -0.7586 & 0.9625 & 0.2711 & -0.4376 & -0.8992 \\
0.6716 & 0.7410 & 0.9519 & -0.3064 & -0.9638 & -0.2666 & 0.4046 & 0.9145 \\
0.6716 & 0.7410 & 0.9519 & -0.3064 & -0.9638 & 0.2666 & 0.4046 & -0.9145 \\
0.6716 & 0.7410 & 0.9519 & -0.3064 & 0.9638 & 0.2666 & 0.4046 & -0.9145 \\
0.6716 & 0.7410 & 0.9519 & -0.3064 & 0.9638 & -0.2666 & 0.4046 & 0.9145 \\
\hline
\end{tabular}

16) F16:

$$
\left\{\begin{array}{l}
4 x_{1}^{3}-3 x_{1}-\cos \left(x_{2}\right)=0 \\
\sin \left(x_{1}^{2}\right)-\left|x_{2}\right|=0
\end{array}\right.
$$

where $x_{i} \in[-2,2], i=1, \cdots, n$, and $n=2$. It has six optimal solutions as shown in Table S-X. This function is modified from [13].

TABLE S-X

THE OPTIMAL SOLUTIONS OF F16

\begin{tabular}{|r|r|}
\hline$x_{1}$ & $x_{2}$ \\
\hline-0.597167 & -0.349098 \\
-0.597167 & 0.349098 \\
-0.442758 & -0.194781 \\
-0.442758 & 0.194781 \\
0.964499 & -0.801774 \\
0.964499 & 0.801774 \\
\hline
\end{tabular}

17) F17:

$\left\{\begin{array}{l}x_{i}+\sum_{j=1}^{n} x_{j}-(n+1)=0 \quad i=1, \cdots, n-1 \\ {\left[\prod_{j=1}^{n} x_{j}\right]-1=0}\end{array}\right.$ where $x_{i} \in[-2,2], i=1, \cdots, n$, and $n=20$. It has two optimal solutions: $(1, \cdots, 1)$ and $(0.994922, \cdots, 0.994922$, 1.101551) [13].

18) F18:

$$
x_{i}-\cos \left(2 x_{i}-\sum_{j=1}^{n} x_{j}\right)=0 \quad i=1, \cdots, n
$$

where $x_{i} \in[-1,1], i=1, \cdots, n$, and $n=3$. It has seven optimal solutions as shown in Table S-XI [14].

TABLE S-XI

THE OPTIMAL SOLUTIONS OF F18

\begin{tabular}{|r|r|r|}
\hline$x_{1}$ & $x_{2}$ & $x_{3}$ \\
\hline 0.810561 & 0.810561 & -0.625687 \\
0.810561 & -0.625687 & 0.810561 \\
-0.625687 & 0.810561 & 0.810561 \\
0.543850 & 0.995778 & 0.543850 \\
0.543850 & 0.543850 & 0.995778 \\
0.995778 & 0.543850 & 0.543850 \\
0.739086 & 0.739086 & 0.739086 \\
\hline
\end{tabular}

19) F19:

$$
\left\{\begin{array}{l}
x_{1}^{2}+x_{2}^{2}-2=0 \\
x_{1}^{2}+x_{2}^{2} / 4-1=0
\end{array}\right.
$$

where $x_{i} \in[-2,2], i=1, \cdots, n$, and $n=2$. It has four optimal solutions as shown in Table S-XII. This function is modified from [15].

TABLE S-XII

THE OPTIMAL SOLUTIONS OF F19

\begin{tabular}{|r|r|}
\hline$x_{1}$ & $x_{2}$ \\
\hline-0.816497 & -1.154701 \\
0.816497 & -1.154701 \\
-0.816497 & 1.154701 \\
0.816497 & 1.154701 \\
\hline
\end{tabular}

20) F20:

$$
\left\{\begin{array}{l}
\exp \left(x_{1}^{2}+x_{2}^{2}\right)-3=0 \\
\left|x_{2}\right|+x_{1}-\sin \left(3\left(\left|x_{2}\right|+x_{1}\right)\right)=0
\end{array}\right.
$$

where $x_{i} \in[-2,2], i=1, \cdots, n$, and $n=2$. It has six optimal solutions as shown in Table S-XIII. This function is modified from [15].

TABLE S-XIII

THE OPTIMAL SOLUTIONS OF F20

\begin{tabular}{|r|r|}
\hline$x_{1}$ & $x_{2}$ \\
\hline-0.741152 & -0.741152 \\
-0.741152 & 0.741152 \\
-0.256625 & 1.016246 \\
-0.256625 & -1.016246 \\
-1.016246 & -0.256625 \\
-1.016246 & 0.256625 \\
\hline
\end{tabular}

21) F21:

$$
\left\{\begin{array}{l}
-3.84 x_{1}^{2}+3.84 x_{1}-x_{2}=0 \\
-3.84 x_{2}^{2}+3.84 x_{2}-x_{3}=0 \\
-3.84 x_{3}^{2}+3.84 x_{3}-x_{1}=0
\end{array}\right.
$$

where $x_{i} \in[0,1], i=1, \cdots, n$, and $n=3$. It has eight optimal solutions as shown in Table S-XIV [16]. 
TABLE S-XIV

THE OPTIMAL SOLUTIONS OF F21

\begin{tabular}{|r|r|r|}
\hline$x_{1}$ & $x_{2}$ & $x_{3}$ \\
\hline 0.000000 & 0.000000 & 0.000000 \\
0.488122 & 0.959435 & 0.149452 \\
0.540304 & 0.953754 & 0.169399 \\
0.959447 & 0.149373 & 0.487917 \\
0.149440 & 0.488092 & 0.959440 \\
0.953781 & 0.169343 & 0.540157 \\
0.169254 & 0.539937 & 0.953788 \\
0.739584 & 0.739584 & 0.739574 \\
\hline
\end{tabular}

22) F22:

$$
\left\{\begin{array}{l}
x_{1}+x_{2}+x_{3}-1=0 \\
x_{1}-x_{2}^{3}=0
\end{array}\right.
$$

where $x_{i} \in[-1,1], i=1, \cdots, n$, and $n=3$. It has infinite optimal solutions [1].

23) F23:

$$
\left\{\begin{array}{l}
x_{1}^{2}+x_{3}^{2}-1=0 \\
x_{2}^{2}+x_{4}^{2}-1=0 \\
x_{5} x_{3}^{3}+x_{6} x_{4}^{3}=0 \\
x_{5} x_{1}^{3}+x_{6} x_{2}^{3}=0 \\
x_{5} x_{1} x_{3}^{2}+x_{6} x_{4}^{2} x_{2}=0 \\
x_{5} x_{1}^{2} x_{3}+x_{6} x_{2}^{2} x_{4}=0
\end{array}\right.
$$

where $x_{i} \in[-1,1], i=1, \cdots, n$, and $n=6$. It has infinite optimal solutions [1], [2].

24) F24:

$$
\left\{\begin{array}{l}
\left(x_{k}+\sum_{i=1}^{n-k-1} x_{i} x_{i+k}\right) x_{n}-c_{k}=0 \quad 1 \leq k \leq n-1 \\
\sum_{i=1}^{n-1} x_{i}+1=0
\end{array}\right.
$$

where $x_{i} \in[-1,1], i=1, \cdots, n, n=20, c_{k}=0$, and $k=1, \cdots, n-1$. It has infinite optimal solutions [1], [2].

\section{5) F25:}

$$
\left\{\begin{array}{l}
x_{2}+2 x_{6}+x_{9}+2 x_{10}-10^{-5}=0 \\
x_{3}+x_{8}-3 \cdot 10^{-5}=0 \\
x_{1}+x_{3}+2 x_{5}+2 x_{8}+x_{9}+x_{10}-5 \cdot 10^{-5}=0 \\
x_{4}+2 x_{7}-10^{-5}=0 \\
0.5140437 \cdot 10^{-7} x_{5}-x_{1}^{2}=0 \\
0.1006932 \cdot 10^{-6} x_{6}-2 x_{2}^{2}=0 \\
0.7816278 \cdot 10^{-15} x_{7}-x_{4}^{2}=0 \\
0.1496236 \cdot 10^{-6} x_{8}-x_{1} x_{3}=0 \\
0.6194411 \cdot 10^{-7} x_{9}-x_{1} x_{2}=0 \\
0.2089296 \cdot 10^{-14} x_{10}-x_{1} x_{2}^{2}=0
\end{array}\right.
$$

where $x_{i} \in[-10,10], i=1, \cdots, n$, and $n=10$. It has infinite optimal solutions [2].

26) F26:

$$
\left\{\begin{array}{l}
3 x_{1}^{2}+\sin \left(x_{1} x_{2}\right)-x_{3}^{2}+2.0=0 \\
2 x_{1}^{3}-x_{2}^{2}-x_{3}+3.0=0 \\
\sin \left(2 x_{1}\right)+\cos \left(x_{2} x_{3}\right)+x_{2}-1.0=0
\end{array}\right.
$$

where $x_{1} \in[-5,5], x_{2} \in[-1,3]$, and $x_{3} \in[-5,5]$. It has two optimal solutions: $(-0.064417,2.090440,-1.370473)$ and $(-0.032759,1.264629,1.400644)$ [17].
27) F27:

$$
\left\{\begin{array}{l}
5 x_{1}^{9}-6 x_{1}^{5} x_{2}^{2}+x_{1} x_{2}^{4}+2 x_{1} x_{3}=0 \\
-2 x_{1}^{6} x_{2}+2 x_{1}^{2} x_{2}^{3}+2 x_{2} x_{3}=0 \\
x_{1}^{2}+x_{2}^{2}-0.265625=0
\end{array}\right.
$$

where $x_{1} \in[-0.6,6], x_{2} \in[-0.6,0.6]$, and $x_{3} \in[-5,5]$. It has 12 optimal solutions as shown in Table S-XV [13], [11].

TABLE S-XV

THE OPTIMAL SOLUTIONS OF F27

\begin{tabular}{|r|r|r|}
\hline$x_{1}$ & $x_{2}$ & $x_{3}$ \\
\hline 0.279855 & 0.432789 & -0.014189 \\
0.279855 & -0.432789 & -0.014189 \\
-0.279855 & 0.432789 & -0.014189 \\
-0.279855 & -0.432789 & -0.014189 \\
0.466980 & 0.218070 & 0.000000 \\
-0.466980 & 0.218070 & 0.000000 \\
0.466980 & -0.218070 & 0.000000 \\
-0.466980 & -0.218070 & 0.000000 \\
0.000000 & 0.515388 & 0.000000 \\
0.000000 & -0.515388 & 0.000000 \\
0.515388 & 0.000000 & -0.012446 \\
-0.515388 & 0.000000 & -0.012446 \\
\hline
\end{tabular}

28) F28:

$$
\left\{\begin{array}{l}
x_{1}^{2}-x_{2}-2=0 \\
x_{1}+\sin \left(\frac{\pi}{2} x_{2}\right)=0
\end{array}\right.
$$

where $x_{1} \in[0,1]$ and $x_{2} \in[-10,0]$. It has two optimal solutions $(0,-2)$ and $(0.707660,-1.5)$ [3].

29) F29:

$$
\left\{\begin{array}{l}
x_{1}^{2}+x_{2}^{2}+x_{1}+x_{2}-8=0 \\
x_{1}\left|x_{2}\right|+x_{1}+\left|x_{2}\right|-5=0
\end{array}\right.
$$

where $x_{1} \in[0,2.5]$ and $x_{2} \in[-4,6]$. It has four optimal solutions $(0.404634,-3.271577),(2.403604,-0.762837),(1$, $2)$, and $(2,1)$. This problem is modified from [18].

30) F30:

$$
\left\{\begin{array}{l}
x_{1}^{2}-\left|x_{2}\right|+1+\frac{1}{9}\left|x_{1}-1\right|=0 \\
x_{2}^{2}+5 x_{1}^{2}-7+\frac{1}{9}\left|x_{2}\right|=0
\end{array}\right.
$$

where $x_{1} \in[-1,1]$ and $x_{2} \in[-10,10]$. It has four optimal solutions $(-0.814326,-1.864719),(0.861828,-1.758100)$, $(-0.814326,1.864719)$, and $(0.861828,1.758100)$. This problem is modified from [18].

31) F31:

$$
\left\{\begin{array}{l}
0.5 \sin \left(x_{1} x_{2}\right)-\frac{0.25}{\pi} x_{2}-0.5 x_{1}=0 \\
\left(1-\frac{0.25}{\pi}\right)\left[\exp \left(2 x_{1}\right)-e\right]+\frac{e}{\pi} x_{2}-2 e x_{1}=0
\end{array}\right.
$$

where $x_{1} \in[0.25,1]$ and $x_{2} \in[1.5,2 \pi]$. It has two optimal solutions $(0.299465,2.836948)$ and $(0.499966$, 3.141589) [12], [19].

32) F32:

$$
\left\{\begin{array}{l}
x_{1}^{x_{2}}+x_{2}^{x_{1}}-5 x_{1} x_{2} x_{3}-85=0 \\
x_{1}^{3}-x_{2}^{x_{3}}-x_{3}^{x_{2}}-60=0 \\
x_{1}^{x_{3}}+x_{3}^{x_{1}}-x_{2}-2=0
\end{array}\right.
$$

where $x_{1} \in[3,5], x_{2} \in[2,4]$, and $x_{3} \in[0.5,2]$. It has one optimal solution $(4,3,1)$ [20]. 
33) F33:

$$
\left\{\begin{array}{l}
x_{1}^{3}-3 x_{1} x_{2}^{2}-1=0 \\
3 x_{1}^{2} x_{2}-x_{2}^{3}+1=0
\end{array}\right.
$$

where $x_{1} \in[-1,-0.1]$ and $x_{2} \in[-2,2]$. It has two optimal solutions $(-0.793701,-0.793701)$ and $(-0.290515$, 1.084215) [9].

\section{4) F34:}

$$
\left\{\begin{array}{l}
0.1 x_{1}+\cos \left(2 x_{2}\right)+0.09240=0 \\
\sin \left(3 x_{3}\right)+\sin \left(\frac{10 x_{1}}{3}\right)+\log \left(2 x_{2}\right)-2.52 x_{3}+0.08805=0 \\
2\left(x_{1}-0.75\right)^{2}+\sin \left(16 \pi x_{2}-\frac{\pi}{2}\right)-3.26815=0
\end{array}\right.
$$

where $x_{1} \in[1,2.5], x_{2} \in[0.2,2]$, and $x_{3} \in[0.1,3]$. It has one optimal solution $(1.852100,0.926050,0.617370)$ [21].

\section{5) F35:}

$$
\left\{\begin{array}{l}
4 x_{1}^{3}-3 x_{1}-x_{2}=0 \\
x_{1}^{2}-x_{2}=0
\end{array}\right.
$$

where $x_{1} \in[-5,1.5]$ and $x_{2} \in[0,5]$. It has three optimal solutions $(-0.75,0.5625),(0,0)$, and $(1,1)[13]$.

\section{6) F36:}

$\left\{\begin{array}{l}x_{1}^{3}-3 x_{1} x_{2}^{2}+a_{1}\left(2 x_{1}^{2}+x_{1} x_{2}\right)+b_{1} x_{2}^{2}+c_{1} x_{1}+a_{2} x_{2}=0 \\ 3 x_{1}^{2} x_{2}-x_{2}^{3}-a_{1}\left(4 x_{1} x_{2}-x_{2}^{2}\right)+b_{2} x_{1}^{2}+c_{2}=0\end{array}\right.$

where $a_{1}=25, b_{1}=1, c_{1}=2, a_{2}=3, b_{2}=4, c_{2}=5$, $x_{1} \in[0,2]$, and $x_{2} \in[10,30]$. It has two optimal solutions $(1.6359718,13.8476653)$ and $(0.6277425,22.2444123)$ [15].

37) F37:

$$
\left\{\begin{array}{l}
x_{1}^{2}-x_{1}-x_{2}^{2}-x_{2}+x_{3}^{2}=0 \\
\sin \left(x_{2}-\exp \left(x_{1}\right)\right)=0 \\
x_{3}-\log \left|x_{2}\right|=0
\end{array}\right.
$$

where $x_{1} \in[0,2], x_{2} \in[-10,10]$, and $x_{3} \in[-1,1]$. It has five optimal solutions shown in Table S-XVI. This problem is modified from [22].

TABLE S-XVI

THE OPTIMAL SOLUTIONS OF F37

\begin{tabular}{|r|r|r|}
\hline$x_{1}$ & $x_{2}$ & $x_{3}$ \\
\hline 0.825297 & -0.859034 & -0.151946 \\
1.299490 & 0.525835 & -0.642769 \\
1.533662 & -1.648068 & 0.499604 \\
1.981360 & -2.172180 & 0.775731 \\
1.983283 & 0.983378 & -0.016762 \\
\hline
\end{tabular}

38) F38:

$$
\left\{\begin{array}{l}
x_{1}^{4}+4 x_{2}^{4}-6.0=0 \\
x_{1}^{2} x_{2}-0.6787=0
\end{array}\right.
$$

where $x_{1} \in[-2,2]$ and $x_{2} \in[0,1.1]$. It has four optimal solutions $(-1.563533,0.277628),(-0.789706,1.088295)$, $(1.563533,0.277628)$, and $(0.789706,1.088295)$. This problem is modified from [23].

\section{REFERENCES}

[1] W. Song, Y. Wang, H.-X. Li, and Z. Cai, "Locating multiple optimal solutions of nonlinear equation systems based on multiobjective optimization," IEEE Trans. on Evol. Comput., vol. 19, no. 3, pp. 414-431, June 2015.

[2] C. Grosan and A. Abraham, "A new approach for solving nonlinear equations systems," IEEE Transactions on Systems, Man and Cybernetics, Part A: Systems and Humans, vol. 38, no. 3, pp. 698-714, May 2008.

[3] E. Pourjafari and H. Mojallali, "Solving nonlinear equations systems with a new approach based on invasive weed optimization algorithm and clustering," Swarm and Evolutionary Computation, vol. 4, no. 0 , pp. $33-43,2012$.

[4] C. A. Floudas, "Recent advances in global optimization for process synthesis, design and control: Enclosure of all solutions," Computers \& Chemical Engineering, vol. 23, Supplement, no. 0, pp. 963 - 973, 1999.

[5] M. J. Hirsch, P. M. Pardalos, and M. G. Resende, "Solving systems of nonlinear equations with continuous GRASP," Nonlinear Analysis: Real World Applications, vol. 10, no. 4, pp. 2000 - 2006, 2009.

[6] N. Henderson, W. F. Sacco, and G. M. Platt, "Finding more than one root of nonlinear equations via a polarization technique: An application to double retrograde vaporization," Chemical Engineering Research and Design, vol. 88, no. 5C6, pp. $551-561,2010$.

[7] A. Morgan and V. Shapiro, "Box-bisection for solving second-degree systems and the problem of clustering," ACM Trans. Math. Softw., vol. 13 , no. 2, pp. 152-167, 1987.

[8] O. E. Turgut, M. S. Turgut, and M. T. Coban, "Chaotic quantum behaved particle swarm optimization algorithm for solving nonlinear system of equations," Computers \& Mathematics with Applications, vol. 68, no. 4, pp. $508-530,2014$.

[9] M. Jaberipour, E. Khorram, and B. Karimi, "Particle swarm algorithm for solving systems of nonlinear equations," Computers \& Mathematics with Applications, vol. 62, no. 2, pp. 566 - 576, 2011.

[10] M. Abdollahi, A. Isazadeh, and D. Abdollahi, "Imperialist competitive algorithm for solving systems of nonlinear equations," Computers \& Mathematics with Applications, vol. 65, no. 12, pp. 1894 - 1908, 2013.

[11] W. Sacco and N. Henderson, "Finding all solutions of nonlinear systems using a hybrid metaheuristic with fuzzy clustering means," Applied Soft Computing, vol. 11, no. 8, pp. 5424 - 5432, 2011.

[12] C. Wang, R. Luo, K. Wu, and B. Han, "A new filled function method for an unconstrained nonlinear equation," Journal of Computational and Applied Mathematics, vol. 235, no. 6, pp. 1689 - 1699, 2011.

[13] R. B. Kearfott, "Some tests of generalized bisection," ACM Trans. Math. Softw., vol. 13, no. 3, pp. 197-220, 1987.

[14] J. R. Sharma and H. Arora, "On efficient weighted-newton methods for solving systems of nonlinear equations," Applied Mathematics and Computation, vol. 222, pp. 497 - 506, 2013.

[15] W. Rheinboldt, "Some nonlinear test problems," $2003 . \quad$ [Online]. Available: http://folk.uib.no/ssu029/Pdf_file/Testproblems/testprobRheinboldt03.pdf

[16] C. Jäger and D. Ratz, "A combined method for enclosing all solutions of nonlinear systems of polynomial equations," Reliable Computing, vol. 1, no. 1, pp. 41-64, 1995.

[17] H. Ren, L. Wu, W. Bi, and I. K. Argyros, "Solving nonlinear equations system via an efficient genetic algorithm with symmetric and harmonious individuals," Applied Mathematics and Computation, vol. 219, no. 23, pp. $10967-10973,2013$.

[18] H. A. e Oliveira Jr. and A. Petraglia, "Solving nonlinear systems of functional equations with fuzzy adaptive simulated annealing," Applied Soft Computing, vol. 13, no. 11, pp. 4349 - 4357, 2013.

[19] C. Floudas, P. Pardalos, C. Adjiman, W. Esposito, Z. Gumus, S. Harding, J. Klepeis, C. Meyer, and C. Schweiger, Handbook of Test Problems in Local and Global Optimization. Kluwer Academic Publishers, 1999.

[20] Y. Mo, H. Liu, and Q. Wang, "Conjugate direction particle swarm optimization solving systems of nonlinear equations," Computers \& Mathematics with Applications, vol. 57, no. 11-12, pp. 1877 - 1882, 2009.

[21] J. Pintér, "Solving nonlinear equation systems via global partition and search: Some experimental results," Computing, vol. 43, no. 4, pp. 309323, 1990.

[22] M. Grau-Sánchez, A. Grau, and M. Noguera, "Frozen divided difference scheme for solving systems of nonlinear equations," J. Comput. Appl. Math., vol. 235, no. 6, pp. 1739-1743, 2011. 
[23] M. Nikkhah-Bahrami and R. Oftadeh, "An effective iterative method for computing real and complex roots of systems of nonlinear equations," Applied Mathematics and Computation, vol. 215, no. 5, pp. 1813 - 1820, 2009. 


\section{S-II. Parameter Settings}

TABLE S-R-I

PARAMETER SETTINGS FOR DIFFERENT METHODS.

\begin{tabular}{|r|l|}
\hline \hline Method & Parameter settings \\
\hline A-WeB & $N P=100, H=N P$ \\
\hline A-MONES & $N P=100, H=N P$ \\
\hline A-MOMMOP & $N P=100, H=N P$ \\
\hline A-MOBiDE & $N P=100, H=N P$ \\
\hline NCDE & $N P=100, F=0.9, C R=0.1$ \\
\hline NSDE & $N P=100, F=0.9, C R=0.1$ \\
\hline LIPS & $N P=100, w=0.729843788$ \\
\hline R3PSO & $N P=100, w=0.729843788, c_{1}=c_{2}=2.05$ \\
\hline Rep-SHADE & $N P=100, H=N P$ \\
\hline Rep-CLPSO & $N P=100, m=7, c=2.0$ \\
\hline jDE-WeB & $N P=100, \tau_{1}=\tau_{2}=0.1$ \\
\hline JADE-WeB & $N P=100, c=0.1, \mu_{C R}=0.5, \mu_{F}=0.5$ \\
\hline
\end{tabular}




\section{S-III. SUPPLEMENTAL RESUlTS}

TABLE S-R-II

COMPARISON OF DIFFERENT METHODS ON TEST INSTANCES F01-F21 WITH RESPECT TO THE PEAK RATIO. THE BEST RESULT FOR EACH TEST INSTANCE AMONG THE COMPARED METHODS IS HIGHLIGHTED IN BOLDFACE.

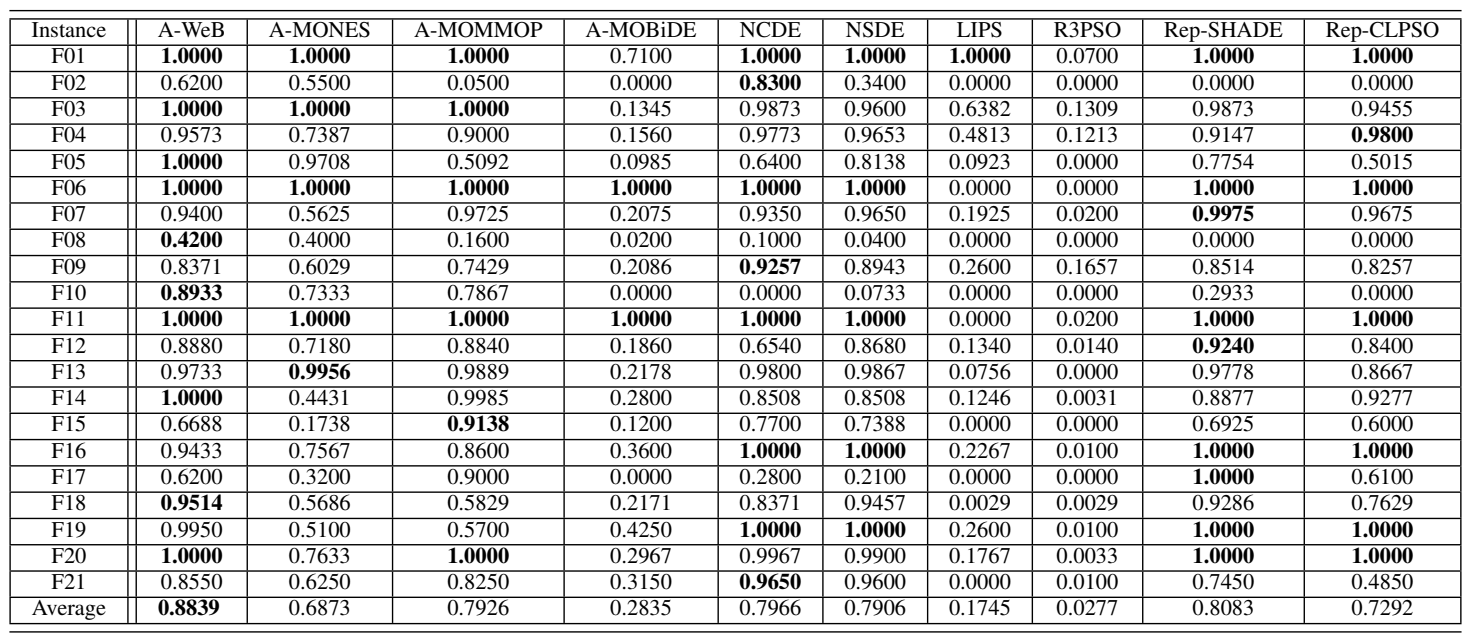

TABLE S-R-III

COMPARISON OF DIFFERENT METHODS ON TEST CASES F01-F21 WITH RESPECT TO THE SUCCESS RATE. THE BEST RESULT FOR EACH TEST INSTANCE AMONG THE COMPARED METHODS IS HIGHLIGHTED IN BOLDFACE.

\begin{tabular}{|c||c|c|c|c|c|c|c|c|c|c|}
\hline \hline Instance & A-WeB & A-MONES & A-MOMMOP & A-MOBiDE & NCDE & NSDE & LIPS & R3PSO & Rep-SHADE & Rep-CLPSO \\
\hline F01 & $\mathbf{1 . 0 0}$ & $\mathbf{1 . 0 0}$ & $\mathbf{1 . 0 0}$ & 0.42 & $\mathbf{1 . 0 0}$ & $\mathbf{1 . 0 0}$ & $\mathbf{1 . 0 0}$ & 0.02 & $\mathbf{1 . 0 0}$ & $\mathbf{1 . 0 0}$ \\
\hline F02 & 0.36 & 0.10 & 0.00 & 0.00 & $\mathbf{0 . 6 8}$ & 0.08 & 0.00 & 0.00 & 0.00 & 0.00 \\
\hline F03 & $\mathbf{1 . 0 0}$ & $\mathbf{1 . 0 0}$ & $\mathbf{1 . 0 0}$ & 0.00 & 0.88 & 0.66 & 0.00 & 0.00 & 0.86 & 0.62 \\
\hline F04 & 0.58 & 0.36 & 0.38 & 0.00 & $\mathbf{0 . 7 2}$ & 0.62 & 0.00 & 0.00 & 0.24 & $\mathbf{0 . 7 2}$ \\
\hline F05 & $\mathbf{1 . 0 0}$ & 0.86 & 0.00 & 0.00 & 0.00 & 0.04 & 0.00 & 0.00 & 0.08 & 0.00 \\
\hline F06 & $\mathbf{1 . 0 0}$ & $\mathbf{1 . 0 0}$ & $\mathbf{1 . 0 0}$ & $\mathbf{1 . 0 0}$ & $\mathbf{1 . 0 0}$ & $\mathbf{1 . 0 0}$ & 0.00 & 0.00 & $\mathbf{1 . 0 0}$ & $\mathbf{1 . 0 0}$ \\
\hline F07 & 0.60 & 0.50 & 0.80 & 0.00 & 0.52 & 0.72 & 0.00 & 0.00 & $\mathbf{0 . 9 8}$ & 0.78 \\
\hline F08 & $\mathbf{0 . 4 2}$ & 0.40 & 0.16 & 0.02 & 0.10 & 0.04 & 0.00 & 0.00 & 0.00 & 0.00 \\
\hline F09 & 0.12 & 0.02 & 0.00 & 0.02 & $\mathbf{0 . 5 0}$ & 0.32 & 0.00 & 0.00 & 0.06 & 0.02 \\
\hline F10 & $\mathbf{0 . 6 8}$ & 0.50 & 0.42 & 0.00 & 0.00 & 0.00 & 0.00 & 0.00 & 0.04 & 0.00 \\
\hline F11 & $\mathbf{1 . 0 0}$ & $\mathbf{1 . 0 0}$ & $\mathbf{1 . 0 0}$ & $\mathbf{1 . 0 0}$ & $\mathbf{1 . 0 0}$ & $\mathbf{1 . 0 0}$ & 0.00 & 0.02 & $\mathbf{1 . 0 0}$ & $\mathbf{1 . 0 0}$ \\
\hline F12 & 0.28 & $\mathbf{0 . 5 0}$ & 0.24 & 0.00 & 0.00 & 0.28 & 0.00 & 0.00 & 0.42 & 0.22 \\
\hline F13 & 0.76 & $\mathbf{0 . 9 6}$ & 0.90 & 0.00 & 0.84 & 0.88 & 0.00 & 0.00 & 0.80 & 0.28 \\
\hline F14 & $\mathbf{1 . 0 0}$ & 0.00 & 0.98 & 0.00 & 0.04 & 0.02 & 0.00 & 0.00 & 0.10 & 0.00 \\
\hline F15 & 0.00 & 0.00 & $\mathbf{0 . 3 6}$ & 0.00 & 0.02 & 0.00 & 0.00 & 0.00 & 0.00 & 0.00 \\
\hline F16 & 0.66 & 0.50 & 0.48 & 0.00 & $\mathbf{1 . 0 0}$ & $\mathbf{1 . 0 0}$ & 0.00 & 0.00 & $\mathbf{1 . 0 0}$ & $\mathbf{1 . 0 0}$ \\
\hline F17 & 0.24 & 0.12 & 0.80 & 0.00 & 0.00 & 0.00 & 0.00 & 0.00 & $\mathbf{1 . 0 0}$ & 0.28 \\
\hline F18 & $\mathbf{0 . 7 0}$ & 0.00 & 0.00 & 0.00 & 0.04 & 0.64 & 0.00 & 0.00 & 0.62 & 0.12 \\
\hline F19 & 0.98 & 0.00 & 0.14 & 0.02 & $\mathbf{1 . 0 0}$ & $\mathbf{1 . 0 0}$ & 0.00 & 0.00 & $\mathbf{1 . 0 0}$ & $\mathbf{1 . 0 0}$ \\
\hline F20 & $\mathbf{1 . 0 0}$ & 0.50 & $\mathbf{1 . 0 0}$ & 0.00 & 0.98 & 0.94 & 0.00 & 0.00 & $\mathbf{1 . 0 0}$ & $\mathbf{1 . 0 0}$ \\
\hline F21 & 0.14 & 0.00 & 0.14 & 0.00 & $\mathbf{0 . 7 4}$ & $\mathbf{0 . 7 4}$ & 0.00 & 0.00 & 0.08 & 0.00 \\
\hline Average & $\mathbf{0 . 6 4}$ & 0.44 & 0.51 & 0.12 & 0.53 & 0.52 & 0.05 & 0.00 & 0.54 & 0.45 \\
\hline
\end{tabular}

TABLE S-R-IV

COMPARISON OF DIFFERENT METHODS ON TEST CASES F26-F38 WITH RESPECT TO THE PEAK RATIO. THE BEST RESULT FOR EACH TEST INSTANCE AMONG THE COMPARED METHODS IS HIGHLIGHTED IN BOLDFACE.

\begin{tabular}{|c|c|c|c|c|c|c|c|}
\hline Instance & A-WeB & A-MONES & A-MOMMOP & NCDE & NSDE & Rep-SHADE & Rep-CLPSO \\
\hline F26 & $\mathbf{1 . 0 0 0 0}$ & 0.9700 & 0.9800 & 0.9900 & 0.9900 & 0.9800 & 0.8100 \\
\hline F27 & 0.0933 & 0.3750 & 0.0000 & 0.6467 & $\mathbf{0 . 7 0 3 3}$ & 0.2733 & 0.4533 \\
\hline F28 & $\mathbf{1 . 0 0 0 0}$ & $\mathbf{1 . 0 0 0 0}$ & $\mathbf{1 . 0 0 0 0}$ & $\mathbf{1 . 0 0 0 0}$ & $\mathbf{1 . 0 0 0 0}$ & $\mathbf{1 . 0 0 0 0}$ & $\mathbf{1 . 0 0 0 0}$ \\
\hline F2 9 & $\mathbf{1 . 0 0 0 0}$ & $\mathbf{1 . 0 0 0 0}$ & $\mathbf{1 . 0 0 0 0}$ & $\mathbf{1 . 0 0 0 0}$ & $\mathbf{1 . 0 0 0 0}$ & $\mathbf{1 . 0 0 0 0}$ & $\mathbf{1 . 0 0 0 0}$ \\
\hline F30 & $\mathbf{1 . 0 0 0 0}$ & 0.7550 & $\mathbf{1 . 0 0 0 0}$ & 0.9700 & $\mathbf{1 . 0 0 0 0}$ & $\mathbf{1 . 0 0 0 0}$ & 0.9900 \\
\hline F31 & $\mathbf{1 . 0 0 0 0}$ & 0.8600 & $\mathbf{1 . 0 0 0 0}$ & $\mathbf{1 . 0 0 0 0}$ & $\mathbf{1 . 0 0 0 0}$ & $\mathbf{1 . 0 0 0 0}$ & 0.9900 \\
\hline F32 & $\mathbf{1 . 0 0 0 0}$ & $\mathbf{1 . 0 0 0 0}$ & $\mathbf{1 . 0 0 0 0}$ & 0.4000 & 0.8400 & $\mathbf{1 . 0 0 0 0}$ & $\mathbf{1 . 0 0 0 0}$ \\
\hline F33 & $\mathbf{1 . 0 0 0 0}$ & $\mathbf{1 . 0 0 0 0}$ & $\mathbf{1 . 0 0 0 0}$ & $\mathbf{1 . 0 0 0 0}$ & 0.9800 & $\mathbf{1 . 0 0 0 0}$ & $\mathbf{1 . 0 0 0 0}$ \\
\hline F34 & 0.8800 & $\mathbf{0 . 9 2 0 0}$ & 0.5200 & 0.1600 & 0.6600 & 0.4200 & 0.1400 \\
\hline F35 & $\mathbf{1 . 0 0 0 0}$ & 0.9467 & 0.9933 & 0.9800 & $\mathbf{1 . 0 0 0 0}$ & $\mathbf{1 . 0 0 0 0}$ & $\mathbf{1 . 0 0 0 0}$ \\
\hline F36 & 0.9400 & 0.9100 & 0.9400 & $\mathbf{1 . 0 0 0 0}$ & $\mathbf{1 . 0 0 0 0}$ & $\mathbf{1 . 0 0 0 0}$ & 0.9200 \\
\hline F37 & 0.9320 & 0.9160 & 0.9000 & 0.7880 & $\mathbf{0 . 9 8 4 0}$ & 0.9480 & 0.3800 \\
\hline F38 & $\mathbf{1 . 0 0 0 0}$ & 0.7600 & $\mathbf{1 . 0 0 0 0}$ & 0.9750 & $\mathbf{1 . 0 0 0 0}$ & $\mathbf{1 . 0 0 0 0}$ & $\mathbf{1 . 0 0 0 0}$ \\
\hline Average & 0.9112 & 0.8779 & 0.8718 & 0.8392 & $\mathbf{0 . 9 3 5 2}$ & 0.8939 & 0.8218 \\
\hline
\end{tabular}


TABLE S-R-V

COMPARISON OF DIFFERENT METHODS ON TEST CASES F26-F38 WITH RESPECT TO THE SUCCESS RATE. THE BEST RESULT FOR EACH TEST INSTANCE AMONG THE COMPARED METHODS IS HIGHLIGHTED IN BOLDFACE.

\begin{tabular}{|c|c|c|c|c|c|c|c|}
\hline Instance & A-WeB & A-MONES & A-MOMMOP & NCDE & NSDE & Rep-SHADE & Rep-CLPSO \\
\hline F26 & $\mathbf{1 . 0 0}$ & 0.94 & 0.96 & 0.98 & 0.98 & 0.96 & 0.62 \\
\hline F27 & 0.00 & 0.00 & 0.00 & 0.00 & $\mathbf{0 . 0 2}$ & 0.00 & 0.00 \\
\hline F28 & $\mathbf{1 . 0 0}$ & $\mathbf{1 . 0 0}$ & $\mathbf{1 . 0 0}$ & $\mathbf{1 . 0 0}$ & $\mathbf{1 . 0 0}$ & $\mathbf{1 . 0 0}$ & $\mathbf{1 . 0 0}$ \\
\hline F29 & $\mathbf{1 . 0 0}$ & $\mathbf{1 . 0 0}$ & $\mathbf{1 . 0 0}$ & $\mathbf{1 . 0 0}$ & $\mathbf{1 . 0 0}$ & $\mathbf{1 . 0 0}$ & $\mathbf{1 . 0 0}$ \\
\hline F30 & $\mathbf{1 . 0 0}$ & 0.50 & $\mathbf{1 . 0 0}$ & 0.88 & $\mathbf{1 . 0 0}$ & $\mathbf{1 . 0 0}$ & 0.96 \\
\hline F31 & $\mathbf{1 . 0 0}$ & 0.74 & $\mathbf{1 . 0 0}$ & $\mathbf{1 . 0 0}$ & $\mathbf{1 . 0 0}$ & $\mathbf{1 . 0 0}$ & 0.98 \\
\hline F32 & $\mathbf{1 . 0 0}$ & $\mathbf{1 . 0 0}$ & $\mathbf{1 . 0 0}$ & 0.40 & 0.84 & $\mathbf{1 . 0 0}$ & $\mathbf{1 . 0 0}$ \\
\hline F33 & $\mathbf{1 . 0 0}$ & $\mathbf{1 . 0 0}$ & $\mathbf{1 . 0 0}$ & $\mathbf{1 . 0 0}$ & 0.96 & $\mathbf{1 . 0 0}$ & $\mathbf{1 . 0 0}$ \\
\hline F34 & 0.88 & $\mathbf{0 . 9 6}$ & 0.52 & 0.16 & 0.66 & 0.42 & 0.14 \\
\hline F35 & $\mathbf{1 . 0 0}$ & 0.86 & 0.98 & 0.96 & $\mathbf{1 . 0 0}$ & $\mathbf{1 . 0 0}$ & $\mathbf{1 . 0 0}$ \\
\hline F36 & 0.88 & 0.84 & 0.88 & $\mathbf{1 . 0 0}$ & $\mathbf{1 . 0 0}$ & $\mathbf{1 . 0 0}$ & 0.84 \\
\hline F37 & 0.66 & 0.58 & 0.54 & 0.18 & $\mathbf{0 . 9 2}$ & 0.74 & 0.00 \\
\hline F38 & $\mathbf{1 . 0 0}$ & 0.50 & $\mathbf{1 . 0 0}$ & 0.90 & $\mathbf{1 . 0 0}$ & $\mathbf{1 . 0 0}$ & $\mathbf{1 . 0 0}$ \\
\hline Average & $\mathbf{0 . 8 8}$ & 0.76 & 0.84 & 0.73 & $\mathbf{0 . 8 8}$ & 0.86 & 0.73 \\
\hline
\end{tabular}

TABLE S-R-VI

INFLUENCE OF THE HISTORICAL MEMORY SIZE $(H)$ ON THE PERFORMANCE OF A-WEB FOR TEST INSTANCES F01-F21 WITH RESPECT TO THE PEAK RATIO. THE BEST RESULT FOR EACH TEST INSTANCE AMONG THE COMPARED METHODS IS HIGHLIGHTED IN BOLDFACE.

\begin{tabular}{|c||c|c|c|c||c||c|c|c|c|}
\hline \hline Instance & $H=5$ & $H=10$ & $H=30$ & $H=50$ & $H=100$ & $H=200$ & $H=300$ & $H=400$ & $H=500$ \\
\hline F01 & $\mathbf{1 . 0 0 0 0}$ & $\mathbf{1 . 0 0 0 0}$ & $\mathbf{1 . 0 0 0 0}$ & 0.9900 & $\mathbf{1 . 0 0 0 0}$ & $\mathbf{1 . 0 0 0 0}$ & $\mathbf{1 . 0 0 0 0}$ & 0.9900 & $\mathbf{1 . 0 0 0 0}$ \\
\hline F02 & 0.0100 & 0.0200 & 0.2400 & 0.4700 & 0.6200 & 0.6900 & 0.6700 & 0.7000 & $\mathbf{0 . 7 3 0 0}$ \\
\hline F03 & $\mathbf{1 . 0 0 0 0}$ & $\mathbf{1 . 0 0 0 0}$ & $\mathbf{1 . 0 0 0 0}$ & $\mathbf{1 . 0 0 0 0}$ & $\mathbf{1 . 0 0 0 0}$ & $\mathbf{1 . 0 0 0 0}$ & $\mathbf{1 . 0 0 0 0}$ & $\mathbf{1 . 0 0 0 0}$ & $\mathbf{1 . 0 0 0 0}$ \\
\hline F04 & 0.9413 & 0.9427 & $\mathbf{0 . 9 6 8 0}$ & 0.9613 & 0.9573 & 0.9533 & 0.9667 & 0.9600 & 0.9627 \\
\hline F05 & 0.9969 & 0.9969 & 0.9969 & 0.9985 & $\mathbf{1 . 0 0 0 0}$ & $\mathbf{1 . 0 0 0 0}$ & $\mathbf{1 . 0 0 0 0}$ & $\mathbf{1 . 0 0 0 0}$ & $\mathbf{1 . 0 0 0 0}$ \\
\hline F06 & $\mathbf{1 . 0 0 0 0}$ & $\mathbf{1 . 0 0 0 0}$ & $\mathbf{1 . 0 0 0 0}$ & $\mathbf{1 . 0 0 0 0}$ & $\mathbf{1 . 0 0 0 0}$ & $\mathbf{1 . 0 0 0 0}$ & $\mathbf{1 . 0 0 0 0}$ & $\mathbf{1 . 0 0 0 0}$ & $\mathbf{1 . 0 0 0 0}$ \\
\hline F07 & 0.8875 & 0.8775 & 0.9250 & 0.9550 & 0.9400 & $\mathbf{0 . 9 8 0 0}$ & 0.9675 & 0.9575 & 0.9450 \\
\hline F08 & 0.3200 & 0.4200 & 0.4000 & 0.4800 & 0.4200 & 0.4600 & 0.4200 & $\mathbf{0 . 5 6 0 0}$ & 0.4800 \\
\hline F09 & 0.7886 & 0.7771 & 0.8257 & 0.8200 & 0.8371 & $\mathbf{0 . 8 4 2 9}$ & 0.8086 & 0.8400 & 0.8171 \\
\hline F10 & 0.3067 & 0.4333 & 0.7533 & 0.8533 & 0.8933 & 0.9467 & $\mathbf{0 . 9 7 3 3}$ & $\mathbf{0 . 9 7 3 3}$ & 0.9533 \\
\hline F11 & $\mathbf{1 . 0 0 0 0}$ & $\mathbf{1 . 0 0 0 0}$ & $\mathbf{1 . 0 0 0 0}$ & $\mathbf{1 . 0 0 0 0}$ & $\mathbf{1 . 0 0 0 0}$ & $\mathbf{1 . 0 0 0 0}$ & $\mathbf{1 . 0 0 0 0}$ & $\mathbf{1 . 0 0 0 0}$ & $\mathbf{1 . 0 0 0 0}$ \\
\hline F12 & 0.8220 & 0.8380 & 0.8860 & 0.8700 & 0.8880 & 0.8940 & $\mathbf{0 . 9 0 4 0}$ & 0.8880 & 0.8720 \\
\hline F13 & $\mathbf{0 . 9 9 3 3}$ & $\mathbf{0 . 9 9 3 3}$ & 0.9844 & 0.9756 & 0.9733 & 0.9756 & 0.9600 & 0.9867 & 0.9689 \\
\hline F14 & $\mathbf{1 . 0 0 0 0}$ & 0.9954 & 0.9985 & 0.9985 & $\mathbf{1 . 0 0 0 0}$ & $\mathbf{1 . 0 0 0 0}$ & $\mathbf{1 . 0 0 0 0}$ & 0.9969 & $\mathbf{1 . 0 0 0 0}$ \\
\hline F15 & 0.5838 & 0.6388 & 0.6875 & 0.7075 & 0.6688 & 0.6938 & 0.7088 & 0.7013 & $\mathbf{0 . 7 2 2 5}$ \\
\hline F16 & 0.9567 & 0.9567 & 0.9600 & 0.9733 & 0.9433 & 0.9567 & 0.9667 & 0.9733 & $\mathbf{0 . 9 7 6 7}$ \\
\hline F17 & 0.5900 & $\mathbf{0 . 6 2 0 0}$ & 0.5800 & 0.5800 & $\mathbf{0 . 6 2 0 0}$ & 0.5500 & 0.5200 & 0.4900 & 0.4900 \\
\hline F18 & 0.8886 & 0.9200 & $\mathbf{0 . 9 7 1 4}$ & 0.9543 & 0.9514 & 0.9514 & 0.9343 & 0.9343 & 0.9400 \\
\hline F19 & $\mathbf{1 . 0 0 0 0}$ & $\mathbf{1 . 0 0 0 0}$ & $\mathbf{1 . 0 0 0 0}$ & $\mathbf{1 . 0 0 0 0}$ & 0.9950 & $\mathbf{1 . 0 0 0 0}$ & $\mathbf{1 . 0 0 0 0}$ & $\mathbf{1 . 0 0 0 0}$ & 0.9950 \\
\hline F20 & 0.9933 & 0.9867 & $\mathbf{1 . 0 0 0 0}$ & $\mathbf{1 . 0 0 0 0}$ & $\mathbf{1 . 0 0 0 0}$ & 0.9967 & $\mathbf{1 . 0 0 0 0}$ & 0.9967 & $\mathbf{1 . 0 0 0 0}$ \\
\hline F21 & $\mathbf{0 . 8 6 5 0}$ & 0.8600 & 0.8225 & 0.8650 & 0.8550 & 0.8575 & 0.8600 & 0.8625 & 0.8400 \\
\hline Average & 0.8068 & 0.8227 & 0.8571 & 0.8787 & 0.8839 & 0.8928 & 0.8886 & $\mathbf{0 . 8 9 5 7}$ & 0.8902 \\
\hline
\end{tabular}

TABLE S-R-VII

INFLUENCE OF THE HISTORICAL MEMORY SIZE $(H)$ ON THE PERFORMANCE OF A-WEB FOR TEST INSTANCES F01-F21 WITH RESPECT TO THE SUCCESS RATE. THE BEST RESULT FOR EACH TEST INSTANCE AMONG THE COMPARED METHODS IS HIGHLIGHTED IN BOLDFACE.

\begin{tabular}{|c||c|c|c|c||c||c|c|c|c|}
\hline \hline Instance & $H=5$ & $H=10$ & $H=30$ & $H=50$ & $H=100$ & $H=200$ & $H=300$ & $H=400$ & $H=500$ \\
\hline F01 & $\mathbf{1 . 0 0}$ & $\mathbf{1 . 0 0}$ & $\mathbf{1 . 0 0}$ & 0.98 & $\mathbf{1 . 0 0}$ & $\mathbf{1 . 0 0}$ & $\mathbf{1 . 0 0}$ & 0.98 & $\mathbf{1 . 0 0}$ \\
\hline F02 & 0.00 & 0.00 & 0.12 & 0.22 & 0.36 & 0.44 & 0.40 & 0.44 & $\mathbf{0 . 5 0}$ \\
\hline F03 & $\mathbf{1 . 0 0}$ & $\mathbf{1 . 0 0}$ & $\mathbf{1 . 0 0}$ & $\mathbf{1 . 0 0}$ & $\mathbf{1 . 0 0}$ & $\mathbf{1 . 0 0}$ & $\mathbf{1 . 0 0}$ & $\mathbf{1 . 0 0}$ & $\mathbf{1 . 0 0}$ \\
\hline F04 & 0.44 & 0.40 & 0.60 & 0.52 & 0.58 & 0.52 & 0.60 & 0.56 & $\mathbf{0 . 6 8}$ \\
\hline F05 & 0.96 & 0.96 & 0.96 & 0.98 & $\mathbf{1 . 0 0}$ & $\mathbf{1 . 0 0}$ & $\mathbf{1 . 0 0}$ & $\mathbf{1 . 0 0}$ & $\mathbf{1 . 0 0}$ \\
\hline F06 & $\mathbf{1 . 0 0}$ & $\mathbf{1 . 0 0}$ & $\mathbf{1 . 0 0}$ & $\mathbf{1 . 0 0}$ & $\mathbf{1 . 0 0}$ & $\mathbf{1 . 0 0}$ & $\mathbf{1 . 0 0}$ & $\mathbf{1 . 0 0}$ & $\mathbf{1 . 0 0}$ \\
\hline F07 & 0.44 & 0.38 & 0.48 & 0.68 & 0.60 & $\mathbf{0 . 8 4}$ & 0.74 & 0.68 & 0.60 \\
\hline F08 & 0.32 & 0.42 & 0.40 & 0.48 & 0.42 & 0.46 & 0.42 & $\mathbf{0 . 5 6}$ & 0.48 \\
\hline F09 & 0.02 & 0.00 & 0.10 & 0.06 & $\mathbf{0 . 1 2}$ & 0.08 & 0.02 & $\mathbf{0 . 1 2}$ & 0.04 \\
\hline F10 & 0.02 & 0.10 & 0.28 & 0.58 & 0.68 & 0.84 & $\mathbf{0 . 9 2}$ & $\mathbf{0 . 9 2}$ & 0.86 \\
\hline F11 & $\mathbf{1 . 0 0}$ & $\mathbf{1 . 0 0}$ & $\mathbf{1 . 0 0}$ & $\mathbf{1 . 0 0}$ & $\mathbf{1 . 0 0}$ & $\mathbf{1 . 0 0}$ & $\mathbf{1 . 0 0}$ & $\mathbf{1 . 0 0}$ & $\mathbf{1 . 0 0}$ \\
\hline F12 & 0.12 & 0.14 & 0.24 & 0.26 & 0.28 & 0.36 & $\mathbf{0 . 4 0}$ & 0.36 & 0.26 \\
\hline F13 & $\mathbf{0 . 9 4}$ & $\mathbf{0 . 9 4}$ & 0.86 & 0.78 & 0.76 & 0.78 & 0.66 & 0.88 & 0.72 \\
\hline F14 & $\mathbf{1 . 0 0}$ & 0.94 & 0.98 & 0.98 & $\mathbf{1 . 0 0}$ & $\mathbf{1 . 0 0}$ & $\mathbf{1 . 0 0}$ & 0.96 & $\mathbf{1 . 0 0}$ \\
\hline F15 & 0.00 & 0.00 & 0.00 & 0.00 & 0.00 & 0.00 & 0.00 & 0.00 & 0.00 \\
\hline F16 & 0.74 & 0.74 & 0.76 & 0.84 & 0.66 & 0.74 & 0.80 & 0.84 & $\mathbf{0 . 8 6}$ \\
\hline F17 & 0.18 & $\mathbf{0 . 2 4}$ & 0.16 & 0.16 & $\mathbf{0 . 2 4}$ & 0.10 & 0.08 & 0.08 & 0.08 \\
\hline F18 & 0.38 & 0.52 & $\mathbf{0 . 8 0}$ & 0.72 & 0.70 & 0.70 & 0.60 & 0.62 & 0.62 \\
\hline F19 & $\mathbf{1 . 0 0}$ & $\mathbf{1 . 0 0}$ & $\mathbf{1 . 0 0}$ & $\mathbf{1 . 0 0}$ & 0.98 & $\mathbf{1 . 0 0}$ & $\mathbf{1 . 0 0}$ & $\mathbf{1 . 0 0}$ & 0.98 \\
\hline F20 & 0.96 & 0.92 & $\mathbf{1 . 0 0}$ & $\mathbf{1 . 0 0}$ & $\mathbf{1 . 0 0}$ & 0.98 & $\mathbf{1 . 0 0}$ & 0.98 & $\mathbf{1 . 0 0}$ \\
\hline F21 & 0.28 & 0.32 & 0.20 & 0.22 & 0.14 & 0.22 & 0.20 & $\mathbf{0 . 3 6}$ & 0.24 \\
\hline Average & 0.56 & 0.57 & 0.62 & 0.64 & 0.64 & 0.67 & 0.66 & $\mathbf{0 . 6 8}$ & 0.66 \\
\hline
\end{tabular}


TABLE S-R-VIII

INFLUENCE OF $F$ AND $C R$ ON THE PERFORMANCE OF A-WEB FOR TEST INSTANCES F01-F2 1 WITH RESPECT TO THE PEAK RATIO. THE BEST RESULT FOR EACH TEST INSTANCE AMONG THE COMPARED METHODS IS HIGHLIGHTED IN BOLDFACE.

\begin{tabular}{|c||c|c|c|c|c|}
\hline \hline Instance & A-WeB & A-WeB-2 & A-WeB-3 & A-WeB-4 & A-WeB-5 \\
\hline F01 & $\mathbf{1 . 0 0 0 0}$ & $\mathbf{1 . 0 0 0 0}$ & $\mathbf{1 . 0 0 0 0}$ & $\mathbf{1 . 0 0 0 0}$ & $\mathbf{1 . 0 0 0 0}$ \\
\hline F02 & $\mathbf{0 . 6 2 0 0}$ & 0.1700 & 0.0000 & 0.5000 & 0.0300 \\
\hline F03 & $\mathbf{1 . 0 0 0 0}$ & 0.9964 & $\mathbf{1 . 0 0 0 0}$ & $\mathbf{1 . 0 0 0 0}$ & $\mathbf{1 . 0 0 0 0}$ \\
\hline F04 & 0.9573 & 0.9013 & 0.9720 & 0.9280 & $\mathbf{0 . 9 8 8 0}$ \\
\hline F05 & $\mathbf{1 . 0 0 0 0}$ & 0.5708 & 0.9846 & $\mathbf{1 . 0 0 0 0}$ & $\mathbf{1 . 0 0 0 0}$ \\
\hline F06 & $\mathbf{1 . 0 0 0 0}$ & $\mathbf{1 . 0 0 0 0}$ & 0.0000 & $\mathbf{1 . 0 0 0 0}$ & $\mathbf{1 . 0 0 0 0}$ \\
\hline F07 & $\mathbf{0 . 9 4 0 0}$ & 0.8650 & 0.6750 & 0.8250 & 0.8225 \\
\hline F08 & 0.4200 & 0.1000 & 0.0200 & 0.4200 & $\mathbf{0 . 9 6 0 0}$ \\
\hline F09 & $\mathbf{0 . 8 3 7 1}$ & 0.8229 & 0.8343 & 0.8286 & 0.8229 \\
\hline F10 & 0.8933 & 0.0333 & 0.8267 & 0.8600 & $\mathbf{0 . 9 0 0 0}$ \\
\hline F11 & $\mathbf{1 . 0 0 0 0}$ & $\mathbf{1 . 0 0 0 0}$ & $\mathbf{1 . 0 0 0 0}$ & $\mathbf{1 . 0 0 0 0}$ & $\mathbf{1 . 0 0 0 0}$ \\
\hline F12 & 0.8880 & 0.8220 & $\mathbf{0 . 9 2 6 0}$ & 0.8720 & 0.9080 \\
\hline F13 & $\mathbf{0 . 9 7 3 3}$ & 0.7489 & 0.7000 & 0.9489 & 0.8756 \\
\hline F14 & $\mathbf{1 . 0 0 0 0}$ & $\mathbf{1 . 0 0 0 0}$ & $\mathbf{1 . 0 0 0 0}$ & $\mathbf{1 . 0 0 0 0}$ & $\mathbf{1 . 0 0 0 0}$ \\
\hline F15 & 0.6688 & 0.5575 & 0.0350 & $\mathbf{0 . 6 8 3 8}$ & 0.5513 \\
\hline F16 & 0.9433 & $\mathbf{0 . 9 5 6 7}$ & 0.9200 & 0.9500 & 0.9233 \\
\hline F17 & $\mathbf{0 . 6 2 0 0}$ & 0.0000 & 0.0000 & 0.0200 & 0.0400 \\
\hline F18 & $\mathbf{0 . 9 5 1 4}$ & 0.7829 & 0.8886 & 0.8371 & 0.8857 \\
\hline F19 & 0.9950 & $\mathbf{1 . 0 0 0 0}$ & $\mathbf{1 . 0 0 0 0}$ & 0.9950 & $\mathbf{1 . 0 0 0 0}$ \\
\hline F20 & $\mathbf{1 . 0 0 0 0}$ & 0.9833 & $\mathbf{1 . 0 0 0 0}$ & 0.9900 & $\mathbf{1 . 0 0 0 0}$ \\
\hline F21 & $\mathbf{0 . 8 5 5 0}$ & 0.7150 & 0.7475 & 0.8425 & 0.7325 \\
\hline Average & $\mathbf{0 . 8 8 3 9}$ & 0.7155 & 0.6919 & 0.8334 & 0.8305 \\
\hline \hline
\end{tabular}

TABLE S-R-IX

INFLUENCE OF $F$ AND $C R$ ON THE PERFORMANCE OF A-WEB FOR TEST INSTANCES F01-F21 With RESPECT TO THE SUCCESS RATE. THE BEST RESULT FOR EACH TEST INSTANCE AMONG THE COMPARED METHODS IS HIGHLIGHTED IN BOLDFACE.

\begin{tabular}{|c||c|c|c|c|c|}
\hline \hline Instance & $\mathrm{A}-\mathrm{WeB}$ & $\mathrm{A}-\mathrm{WeB}-2$ & $\mathrm{~A}-\mathrm{WeB}-3$ & $\mathrm{~A}-\mathrm{WeB}-4$ & $\mathrm{~A}-\mathrm{WeB}-5$ \\
\hline F01 & $\mathbf{1 . 0 0}$ & $\mathbf{1 . 0 0}$ & $\mathbf{1 . 0 0}$ & $\mathbf{1 . 0 0}$ & $\mathbf{1 . 0 0}$ \\
\hline F02 & $\mathbf{0 . 3 6}$ & 0.06 & 0.00 & 0.12 & 0.00 \\
\hline F03 & $\mathbf{1 . 0 0}$ & 0.96 & $\mathbf{1 . 0 0}$ & $\mathbf{1 . 0 0}$ & $\mathbf{1 . 0 0}$ \\
\hline F04 & 0.58 & 0.26 & 0.68 & 0.30 & $\mathbf{0 . 8 2}$ \\
\hline F05 & $\mathbf{1 . 0 0}$ & 0.00 & 0.86 & $\mathbf{1 . 0 0}$ & $\mathbf{1 . 0 0}$ \\
\hline F06 & $\mathbf{1 . 0 0}$ & $\mathbf{1 . 0 0}$ & 0.00 & $\mathbf{1 . 0 0}$ & $\mathbf{1 . 0 0}$ \\
\hline F07 & $\mathbf{0 . 6 0}$ & 0.00 & 0.26 & 0.20 & 0.18 \\
\hline F08 & 0.42 & 0.10 & 0.02 & 0.42 & $\mathbf{0 . 9 6}$ \\
\hline F09 & 0.12 & 0.06 & $\mathbf{0 . 1 4}$ & 0.08 & 0.12 \\
\hline F10 & 0.68 & 0.00 & 0.52 & 0.62 & $\mathbf{0 . 7 0}$ \\
\hline F11 & $\mathbf{1 . 0 0}$ & $\mathbf{1 . 0 0}$ & $\mathbf{1 . 0 0}$ & $\mathbf{1 . 0 0}$ & $\mathbf{1 . 0 0}$ \\
\hline F12 & 0.28 & 0.08 & $\mathbf{0 . 4 4}$ & 0.22 & 0.32 \\
\hline F13 & $\mathbf{0 . 7 6}$ & 0.04 & 0.02 & 0.60 & 0.16 \\
\hline F14 & $\mathbf{1 . 0 0}$ & $\mathbf{1 . 0 0}$ & $\mathbf{1 . 0 0}$ & $\mathbf{1 . 0 0}$ & $\mathbf{1 . 0 0}$ \\
\hline F15 & 0.00 & 0.00 & 0.00 & 0.00 & 0.00 \\
\hline F16 & 0.66 & $\mathbf{0 . 7 6}$ & 0.52 & 0.70 & 0.58 \\
\hline F17 & $\mathbf{0 . 2 4}$ & 0.00 & 0.00 & 0.00 & 0.00 \\
\hline F18 & $\mathbf{0 . 7 0}$ & 0.10 & 0.22 & 0.12 & 0.30 \\
\hline F19 & 0.98 & $\mathbf{1 . 0 0}$ & $\mathbf{1 . 0 0}$ & 0.98 & $\mathbf{1 . 0 0}$ \\
\hline F20 & $\mathbf{1 . 0 0}$ & 0.90 & $\mathbf{1 . 0 0}$ & 0.94 & $\mathbf{1 . 0 0}$ \\
\hline F21 & 0.14 & 0.00 & 0.04 & $\mathbf{0 . 1 8}$ & 0.06 \\
\hline Average & $\mathbf{0 . 6 4}$ & 0.40 & 0.46 & 0.55 & 0.58 \\
\hline \hline
\end{tabular}


TABLE S-R-X

INFLUENCE OF THE PARAMETER ADAPTATION ON THE PERFORMANCE OF A-WEB FOR TEST INSTANCES F01-F21 WITH RESPECT TO THE PEAK RATIO. THE BEST RESULT FOR EACH TEST INSTANCE AMONG THE COMPARED METHODS IS HIGHLIGHTED IN BOLDFACE.

\begin{tabular}{|c||c|c|c|}
\hline \hline Instance & A-WeB & $j D E-W e B$ & $J A D E-W e B$ \\
\hline F01 & $\mathbf{1 . 0 0 0 0}$ & $\mathbf{1 . 0 0 0 0}$ & $\mathbf{1 . 0 0 0 0}$ \\
\hline F02 & 0.6200 & 0.3600 & $\mathbf{0 . 7 0 0 0}$ \\
\hline F03 & $\mathbf{1 . 0 0 0 0}$ & $\mathbf{1 . 0 0 0 0}$ & $\mathbf{1 . 0 0 0 0}$ \\
\hline F04 & 0.9573 & 0.9467 & $\mathbf{0 . 9 6 5 3}$ \\
\hline F05 & $\mathbf{1 . 0 0 0 0}$ & 0.9969 & $\mathbf{1 . 0 0 0 0}$ \\
\hline F06 & $\mathbf{1 . 0 0 0 0}$ & $\mathbf{1 . 0 0 0 0}$ & $\mathbf{1 . 0 0 0 0}$ \\
\hline F07 & 0.9400 & 0.8700 & $\mathbf{0 . 9 7 2 5}$ \\
\hline F08 & 0.4200 & $\mathbf{0 . 7 2 0 0}$ & 0.1800 \\
\hline F09 & 0.8371 & 0.8086 & $\mathbf{0 . 8 5 1 4}$ \\
\hline F10 & 0.8933 & $\mathbf{0 . 9 4 6 7}$ & 0.2467 \\
\hline F11 & $\mathbf{1 . 0 0 0 0}$ & $\mathbf{1 . 0 0 0 0}$ & $\mathbf{1 . 0 0 0 0}$ \\
\hline F12 & 0.8880 & 0.8240 & $\mathbf{0 . 9 0 8 0}$ \\
\hline F13 & $\mathbf{0 . 9 7 3 3}$ & 0.9667 & 0.9444 \\
\hline F14 & $\mathbf{1 . 0 0 0 0}$ & $\mathbf{1 . 0 0 0 0}$ & $\mathbf{1 . 0 0 0 0}$ \\
\hline F15 & 0.6688 & 0.4938 & $\mathbf{0 . 7 3 6 3}$ \\
\hline F16 & 0.9433 & 0.9467 & $\mathbf{0 . 9 6 0 0}$ \\
\hline F17 & $\mathbf{0 . 6 2 0 0}$ & 0.5900 & 0.2900 \\
\hline F18 & $\mathbf{0 . 9 5 1 4}$ & 0.8600 & 0.8629 \\
\hline F19 & 0.9950 & 0.9950 & $\mathbf{1 . 0 0 0 0}$ \\
\hline F20 & $\mathbf{1 . 0 0 0 0}$ & $\mathbf{1 . 0 0 0 0}$ & $\mathbf{1 . 0 0 0 0}$ \\
\hline F21 & 0.8550 & 0.7650 & $\mathbf{0 . 8 5 7 5}$ \\
\hline Average & $\mathbf{0 . 8 8 3 9}$ & 0.8614 & 0.8321 \\
\hline \hline
\end{tabular}

TABLE S-R-XI

INFLUENCE OF THE PARAMETER ADAPTATION ON THE PERFORMANCE OF A-WEB FOR TEST INSTANCES F01-F21 WITH RESPECT TO THE SUCCESS RATE. THE BEST RESULT FOR EACH TEST INSTANCE AMONG THE COMPARED METHODS IS HIGHLIGHTED IN BOLDFACE.

\begin{tabular}{|c||c|c|c|}
\hline \hline Instance & $\mathrm{A}-\mathrm{WeB}$ & $\mathrm{j} D \mathrm{DE}-\mathrm{WeB}$ & $\mathrm{J} A \mathrm{DE}-\mathrm{WeB}$ \\
\hline F01 & $\mathbf{1 . 0 0}$ & $\mathbf{1 . 0 0}$ & $\mathbf{1 . 0 0}$ \\
\hline F02 & 0.36 & 0.10 & $\mathbf{0 . 4 6}$ \\
\hline F03 & $\mathbf{1 . 0 0}$ & $\mathbf{1 . 0 0}$ & $\mathbf{1 . 0 0}$ \\
\hline F04 & 0.58 & 0.48 & $\mathbf{0 . 6 0}$ \\
\hline F05 & $\mathbf{1 . 0 0}$ & 0.96 & $\mathbf{1 . 0 0}$ \\
\hline F06 & $\mathbf{1 . 0 0}$ & $\mathbf{1 . 0 0}$ & $\mathbf{1 . 0 0}$ \\
\hline F07 & 0.60 & 0.28 & $\mathbf{0 . 8 6}$ \\
\hline F08 & 0.42 & $\mathbf{0 . 7 2}$ & 0.18 \\
\hline F09 & $\mathbf{0 . 1 2}$ & 0.04 & $\mathbf{0 . 1 2}$ \\
\hline F10 & 0.68 & $\mathbf{0 . 8 4}$ & 0.06 \\
\hline F11 & $\mathbf{1 . 0 0}$ & $\mathbf{1 . 0 0}$ & $\mathbf{1 . 0 0}$ \\
\hline F12 & 0.28 & 0.14 & $\mathbf{0 . 4 2}$ \\
\hline F13 & $\mathbf{0 . 7 6}$ & 0.74 & 0.52 \\
\hline F14 & $\mathbf{1 . 0 0}$ & $\mathbf{1 . 0 0}$ & $\mathbf{1 . 0 0}$ \\
\hline F15 & 0.00 & 0.00 & $\mathbf{0 . 0 2}$ \\
\hline F16 & 0.66 & 0.68 & $\mathbf{0 . 7 6}$ \\
\hline F17 & $\mathbf{0 . 2 4}$ & 0.04 & 0.04 \\
\hline F18 & $\mathbf{0 . 7 0}$ & 0.22 & 0.24 \\
\hline F19 & 0.98 & 0.98 & $\mathbf{1 . 0 0}$ \\
\hline F20 & $\mathbf{1 . 0 0}$ & $\mathbf{1 . 0 0}$ & $\mathbf{1 . 0 0}$ \\
\hline F21 & 0.14 & 0.04 & $\mathbf{0 . 2 4}$ \\
\hline Average & $\mathbf{0 . 6 4}$ & 0.58 & 0.60 \\
\hline \hline
\end{tabular}

TABLE S-R-XII

COMPARISON BETWEEN “DE/CURRENT/1" AND “DE/RAND/1" IN A-WEB. THE BETTER RESUlt FOR EACH TEST INSTANCE BETWEEN THE COMPARED METHODS IS HIGHLIGHTED IN BOLDFACE. IN THE LAST ROW, THE RESULTS IN THE FORM OF $\left(R^{+}, R^{-}, p\right)$ ARE OBTAINED BY THE MULTIPLE-PROBLEM WILCOXON TEST.

\begin{tabular}{|c||c|c||c|c|}
\hline \hline \multirow{2}{*}{ Instance } & \multicolumn{2}{|c||}{$P R$} & \multicolumn{2}{c|}{$S R$} \\
\cline { 2 - 5 } & DE/current/1 & DE/rand/1 & DE/current/1 & DE/rand/1 \\
\hline F02 & 0.6200 & $\mathbf{0 . 9 1 0 0}$ & 0.36 & $\mathbf{0 . 8 2}$ \\
\hline F04 & 0.9573 & $\mathbf{0 . 9 7 6 0}$ & 0.58 & $\mathbf{0 . 7 0}$ \\
\hline F05 & $\mathbf{1 . 0 0 0 0}$ & 0.9662 & $\mathbf{1 . 0 0}$ & 0.66 \\
\hline F07 & $\mathbf{0 . 9 4 0 0}$ & 0.9075 & $\mathbf{0 . 6 0}$ & 0.52 \\
\hline F08 & $\mathbf{0 . 4 2 0 0}$ & 0.2400 & $\mathbf{0 . 4 2}$ & 0.24 \\
\hline F09 & $\mathbf{0 . 8 3 7 1}$ & 0.8114 & $\mathbf{0 . 1 2}$ & 0.08 \\
\hline F10 & $\mathbf{0 . 8 9 3 3}$ & 0.7133 & $\mathbf{0 . 6 8}$ & 0.14 \\
\hline F12 & 0.8880 & $\mathbf{0 . 9 0 6 0}$ & 0.28 & $\mathbf{0 . 3 4}$ \\
\hline F13 & $\mathbf{0 . 9 7 3 3}$ & 0.7333 & $\mathbf{0 . 7 6}$ & 0.04 \\
\hline F15 & $\mathbf{0 . 6 6 8 8}$ & 0.6438 & 0.00 & 0.00 \\
\hline F16 & $\mathbf{0 . 9 4 3 3}$ & 0.8433 & $\mathbf{0 . 6 6}$ & 0.30 \\
\hline F17 & 0.6200 & $\mathbf{0 . 6 8 0 0}$ & 0.24 & $\mathbf{0 . 3 6}$ \\
\hline F18 & $\mathbf{0 . 9 5 1 4}$ & 0.9171 & $\mathbf{0 . 7 0}$ & 0.48 \\
\hline F19 & 0.9950 & $\mathbf{1 . 0 0 0 0}$ & 0.98 & $\mathbf{1 . 0 0}$ \\
\hline F21 & $\mathbf{0 . 8 5 5 0}$ & 0.6375 & $\mathbf{0 . 1 4}$ & 0.04 \\
\hline Average & $\mathbf{0 . 8 3 7 5}$ & 0.7924 & $\mathbf{0 . 5 0}$ & 0.38 \\
\hline Wilcoxon test & $(90.0,30.0,9.46 \mathrm{E}-02)$ & $(83.5,36.5$ & $1.53 \mathrm{E}-01)$ \\
\hline \hline
\end{tabular}


TABLE S-R-XIII

INFLUENCE OF THE DISTANCE COMPARISON CRITERION FOR A-WEB. THE BETTER RESULT FOR EACH TEST INSTANCE BETWEEN THE COMPARED METHODS IS HIGHLIGHTED IN BOLDFACE. IN THE LAST ROW, THE RESULTS IN THE FORM OF $\left(R^{+}, R^{-}, p\right)$ ARE OBTAINED BY THE MULTIPLE-PROBLEM WILCOXON TEST.

\begin{tabular}{|c||c|c||c|c|}
\hline \multirow{2}{*}{ Instance } & \multicolumn{2}{|c||}{$P R$} & \multicolumn{2}{c|}{$S R$} \\
\cline { 2 - 5 } & A-WeB & A-WeB-6 & A-WeB & A-WeB-6 \\
\hline F02 & 0.6200 & $\mathbf{0 . 6 3 0 0}$ & 0.36 & $\mathbf{0 . 4 6}$ \\
\hline F04 & 0.9573 & $\mathbf{0 . 9 6 8 0}$ & 0.58 & $\mathbf{0 . 6 6}$ \\
\hline F05 & $\mathbf{1 . 0 0 0 0}$ & 0.7354 & $\mathbf{1 . 0 0}$ & 0.10 \\
\hline F07 & 0.9400 & $\mathbf{0 . 9 9 2 5}$ & 0.60 & $\mathbf{0 . 9 4}$ \\
\hline F08 & $\mathbf{0 . 4 2 0 0}$ & 0.2800 & $\mathbf{0 . 4 2}$ & 0.28 \\
\hline F09 & 0.8371 & $\mathbf{0 . 8 7 4 3}$ & 0.12 & $\mathbf{0 . 1 8}$ \\
\hline F10 & 0.8933 & $\mathbf{0 . 9 8 0 0}$ & 0.68 & $\mathbf{0 . 9 4}$ \\
\hline F12 & 0.8880 & $\mathbf{0 . 9 3 0 0}$ & 0.28 & $\mathbf{0 . 5 6}$ \\
\hline F13 & $\mathbf{0 . 9 7 3 3}$ & 0.9711 & $\mathbf{0 . 7 6}$ & 0.74 \\
\hline F14 & $\mathbf{1 . 0 0 0 0}$ & 0.9985 & $\mathbf{1 . 0 0}$ & 0.98 \\
\hline F15 & 0.6688 & $\mathbf{0 . 7 7 1 3}$ & 0.00 & 0.00 \\
\hline F16 & 0.9433 & $\mathbf{0 . 9 8 6 7}$ & 0.66 & $\mathbf{0 . 9 2}$ \\
\hline F17 & 0.6200 & $\mathbf{0 . 7 0 0 0}$ & 0.24 & $\mathbf{0 . 4 0}$ \\
\hline F18 & 0.9514 & $\mathbf{0 . 9 7 7 1}$ & 0.70 & $\mathbf{0 . 8 4}$ \\
\hline F19 & 0.9950 & $\mathbf{1 . 0 0 0 0}$ & 0.98 & $\mathbf{1 . 0 0}$ \\
\hline F21 & 0.8550 & $\mathbf{0 . 9 8 0 0}$ & 0.14 & $\mathbf{0 . 8 6}$ \\
\hline Average & 0.8477 & $\mathbf{0 . 8 6 0 9}$ & 0.53 & $\mathbf{0 . 6 2}$ \\
\hline Wilcoxon test & $(34.0,102.0, \geq 0.2)$ & $(34.0,102.0, \geq 0.2)$ \\
\hline \hline
\end{tabular}

